\title{
County-Level Estimates of Excess Mortality Associated with COVID-19 in the United States
}

\author{
Calvin A. Ackley*1, Dielle J. Lundberg ${ }^{2}$, Lei $\mathrm{Ma}^{3}$, Irma T. Elo ${ }^{4}$, Samuel H. Preston ${ }^{4}$, \\ and Andrew C. Stokes ${ }^{2}$ \\ ${ }^{1}$ U.S. Bureau of Economic Analysis, Washington, D.C. \\ ${ }^{2}$ Department of Global Health, Boston University School of Public Health, Boston, MA \\ ${ }^{3}$ Department of Economics, Boston University, Boston, MA \\ ${ }^{4}$ Department of Sociology, University of Pennsylvania, Philadelphia, PA
}

August 27, 2021

\begin{abstract}
Background: The COVID-19 pandemic in the U.S. has been largely monitored on the basis of death certificates containing reference to COVID-19. However, prior analyses reveal that a significant percentage of excess deaths associated with the pandemic were not directly assigned to COVID-19.

Methods: In the present study, we estimate a generalized linear model of expected mortality in 2020 based on historical trends in deaths by county of residence between 2011 and 2019. We use the results of the model to generate estimates of excess mortality and excess deaths not assigned to COVID-19 for 1,470 county-sets in the U.S. representing 3,138 counties.

Results: During 2020, more than one-fourth of U.S. residents (91.2 million) lived in counties where less than $75 \%$ of excess deaths were assigned to COVID-19. Across the country, we estimated that 439,698 excess deaths occurred in 2020, among which 86.7\% were assigned to COVID-19. Some regions (Mideast, Great Lakes, New England, and Far West) reported the most excess deaths in large central metros, whereas other regions (Southwest, Southeast, Plains, and Rocky Mountains) reported the highest excess mortality in nonmetro areas. The proportion assigned to COVID-19 was lowest in large central metro areas $(79.3 \%)$ compared to medium or small metros $(87.4 \%)$, nonmetro areas $(89.4 \%)$ and large fringe metros $(95.2 \%)$. Regionally, the proportion of excess deaths assigned to COVID-19 was lowest in the Southeast (81.1\%), Far West (81.2\%), Southwest (82.6\%), and Rocky Mountains (85.2\%). Across the regions, the number of excess deaths exceeded the number of directly assigned COVID-19 deaths in the majority of counties. The exception to this was in New England, which reported more directly assigned COVID-19 deaths than excess deaths in large central metro areas, large fringe metros, and medium or small metros.

Conclusions: Across the U.S., many counties had substantial numbers of excess deaths that were not accounted for in direct COVID-19 death counts. Estimates of excess mortality at the local level can inform the allocation of resources to areas most impacted by the pandemic and contribute to positive protective behavior feedback loops (i.e. increases in mask-wearing and vaccine uptake).
\end{abstract}

${ }^{*}$ The authors would like to thank Robert N. Anderson and Farida B. Ahmad from the National Center for Health Statistics, Katherine Hempstead from the Robert Wood Johnson Foundation (RWJF), and Abe Dunn from the Bureau of Economic Analysis (BEA) for their input and technical support. Stokes gratefully acknowledges financial support from the RWJF. The views expressed in this paper are those of the authors and not necessarily the views of the BEA or RWJF. 


\section{Introduction}

Estimates of excess deaths are critical to tracking the direct and indirect effects of the COVID19 pandemic and for developing equitable policy responses.[1] Provisional estimates from the Center for Disease Control and Prevention (CDC) indicate that between 545,600 and 660,200 excess deaths occurred in the United States from January 26, 2020 to February 27, 2021.[2] The CDC further estimates that between 75 and $88 \%$ of excess deaths were directly assigned to COVID-19 on death certificates, suggesting that between 12 and $25 \%$ of excess deaths were not assigned to COVID-19.[2] Other prior estimates of excess mortality have also found significant discrepancies between direct COVID-19 deaths and excess mortality. $[2,3,4,5]$

Excess deaths not assigned to COVID-19 may reflect a variety of factors, including COVID-19 deaths that were ascribed to other causes of death due to limited testing, [6] indirect deaths caused by interruptions in the provision of health care services, $[7,8]$ or indirect deaths caused by the broader social and economic consequences of the pandemic. $[9,10,11]$ At the state-level, the percent of excess deaths not assigned to COVID-19 has been shown to vary significantly, suggesting that attribution of deaths to COVID-19 may not be uniform across the country.[4]

While prior estimates of excess mortality at the national and state levels are useful for understanding the impact of the pandemic on mortality levels broadly, estimation of excess mortality at the county-level may be valuable for several reasons. First, states are heterogeneous units in respect to sociodemographic characteristics, which contributes to geographic differences in mortality.[12, 13] Prior studies have found that the proportion of excess deaths not assigned to COVID-19 differed significantly by county-level sociodemographic and health care factors.[5, 14] Second, deaths are registered at the county-level.[15] Thus, it is reasonable to assume that administrative differences may exist between counties in the processing and assignment of deaths. Third, county-level data are essential for informing community and policy interventions. If a county's direct COVID-19 tallies are substantially underestimated, measuring excess mortality at the county-level may be an important step to appreciating the full burden of the COVID-19 pandemic in an area and allocating response resources appropriately. Fourth, providing accurate data to residents could result in a positive behavior feedback loop, which encourages residents to understand the extent of mortality in their area and take protective actions such as wearing masks and pursuing vaccination.[16]

The objective of the present study is to generate estimates of excess mortality at the county-level and examine geographic variation in excess mortality and the proportion of excess deaths not assigned to COVID-19. Examining excess deaths at the county-level has the potential to identify counties with high excess mortality but low directly assigned COVID-19 mortality, indicating that COVID-19 is underreported on death certificates or substantial numbers of indirect deaths have occurred in these areas. These counties could represent regions that have been especially hard hit by the COVID-19 pandemic but whose mortality impacts did not appear in direct COVID-19 tallies and whose excess mortality has thus been hidden. In estimating excess mortality at the county-level, this study seeks to provide communities with estimates of the severity of the pandemic in their area which can be used to inform pandemic preparedness and response at the county, state, and national levels.

\section{Data}

We used provisional data from the National Center for Health Statistics (NCHS) on COVID-19 mortality and all-cause mortality by county of residence from January 1 to December 31, 2020 reported by 
June $3,2021{ }^{1}$ We used data with a twenty-two week lag (December 31, 2020 to June 3, 2021) to improve the completeness of data, since prior analysis of provisional NCHS vital statistics reveal low completeness within the month following a death but more than 75 percent completeness after eight-weeks.[17] For counties with between 1 and 9 all-cause or COVID-19 deaths, NCHS censored the exact number of deaths, so we used the median value (5).

COVID-19 deaths were identified using the International Statistical Classification of Diseases and Related Health Problems, Tenth Revision (ICD-10) code U07.1 and included deaths assigned to COVID19 as the underlying cause as well as deaths in which COVID-19 was reported as a cause that contributed to death on the death certificate. Prior reports indicate that COVID-19 was assigned as the underlying cause on death certificate in $92 \%$ of deaths.[18]

For our historical comparison period, we used CDC Wonder data on all-cause mortality by county of residence from 2011 to 2019. For counties with fewer than 10 deaths, CDC Wonder censored the exact number of deaths, so we used a value of 5 . To compute death rates, we used data on estimated populations from the U.S. Census Bureau for the years 2011 through 2020.

To assess geographic patterns in mortality, we classified counties into metropolitan-nonmetropolitan categories (large central metro, large fringe metro, small/medium metro, and nonmetro)[19]. The classifications were developed by the US Department of Agriculture (USDA) Economic Research Service (ERS) and modified by the National Center for Health Statistics. Large central metros included counties in metropolitan statistical areas with a population of more than 1 million. Large fringe metros were counties that surrounded the large central metros. Small or medium metros included counties in metropolitan statistical areas with a population between 50,000 and 999,999. Nonmetropolitan areas included all other counties. We also examined patterns by Bureau of Economic Analysis (BEA) Regions. These included New England (Connecticut, Maine, Massachusetts, New Hampshire, Rhode Island, and Vermont), Mideast (Delaware, District of Columbia, Maryland, New Jersey, New York, and Pennsylvania), Great Lakes (Illinois, Indiana, Michigan, Ohio, and Wisconsin), Plains (Iowa, Kansas, Minnesota, Missouri, Nebraska, North Dakota, and South Dakota); Southeast (Alabama, Arkansas, Florida, Georgia, Kentucky, Louisiana, Mississippi, North Carolina, South Carolina, Tennessee, Virginia, and West Virginia), Southwest (Arizona, New Mexico, Oklahoma, and Texas), Rocky Mountain (Colorado, Idaho, Montana, Utah, and Wyoming), and Far West (Alaska, California, Hawaii, Nevada, Oregon, and Washington). We then stratified these regions by metropolitan-nonmetropolitan categories to yield 32 distinct geographic units.

In order to provide precise estimates reflecting each county area, we aggregated small counties according to the United States Census Bureau's County-Sets. ${ }^{2}$ Counties with a population of 50,000 or more generally stood alone, and smaller groups of counties were combined to form sets with a total population exceeding 50,000. Importantly, these county-sets were contiguous and preserved state borders. In using these county-sets, we were able to provide more precise estimates for geographies of sparsely populated counties than if we has modelled each of these small counties separately. Our data included 3138 counties with reported all-cause mortality for 2011-2020. This translated into a final analytic sample of 1,470 county sets.

The present investigation relied on de-identified publicly available data and was therefore exempted from review by the Boston University Medical Center Institutional Review Board. Analyses were conducted using R/R Studio. Additional details about the data along with programming code for replicating

\footnotetext{
${ }^{1}$ Data can be accessed at: https://data.cdc.gov/NCHS/AH-County-of-Residence-COVID-19-Deaths-Counts-2020/75vbd79q

${ }^{2}$ Detailed information on Census county-sets can be found at https://www.census.gov/geographies/referencefiles/2000/demo/eeo/county-sets.html
} 
the analyses of the present study are available from the linked GitHub repository. ${ }^{3}$

\section{Methodology}

\subsection{General Model for County-Level Mortality}

To generate a prediction of expected mortality in 2020, we estimated a statistical model of mortality using historical mortality data from 2011-2019. Specifically, we modeled mortality at the county-set-year level using a quasi-poisson generalized linear model (QP-GLM) of the following form: ${ }^{45}$

$$
E\left[Y_{i t} \mid \mathbf{Z}_{\mathbf{i t}}\right]=\exp \left(\alpha_{i}+\phi Y_{t-1}+\delta t+\beta_{i} t\right)
$$

Here, $Y_{i t}$ denoted the number of all-cause deaths divided by the total population of county $i$ in year $t$. In the linear index, we included a county-set-specific intercept term, $\alpha_{i}$, which captured latent characteristics of each county-set that may be correlated with mortality. Importantly, this term picked up relevant information such as the distribution of age and health in each county-set. ${ }^{6}$ We included one lag of the dependent variable, $Y_{t-1}$, to capture potential serial correlation in mortality. ${ }^{7}$ We included a time trend, $t$, and allowed the time trend to vary across county-sets according to $\beta_{i}$. This accounts for the evolving distribution of age and other demographic characteristics across granular geographies that may be related to mortality.

We used the model estimates described above to compute fitted values of total deaths and the death rate per 100,000 person-years for each county-set and year from 2011-2020. ${ }^{8}$

\subsection{Excess Deaths}

We defined excess deaths as the difference between the number of predicted all-cause deaths in 2020 and the number of observed all-cause deaths in 2020. For each county in our sample, we produced an excess death rate for 2020 as well as a ratio of observed to expected deaths.

\subsection{Excess Deaths Not Assigned to COVID-19}

We defined excess deaths not assigned to COVID-19 as the difference between the number of excess deaths in 2020 and the number of observed directly assigned COVID-19 deaths in 2020. For each county in our sample, we decomposed the excess death rate into: (1) the observed death rate from COVID-19 and (2) the excess death rate not assigned to COVID-19. Next, we defined the proportion of excess deaths assigned to COVID-19 as the ratio of the direct COVID-19 death rate to the excess death rate.

\footnotetext{
${ }^{3}$ The GitHub repository can be accessed at: https://github.com/pophealthdeterminantslab/county-level-estimates-ofexcess-mortality

${ }^{4}$ See, for example, McCullaugh and Nelder (1989)[20]

${ }^{5}$ Details on how our exact specification was chosen are given in Appendix B

${ }^{6}$ It may be desirable to explicitly include measures of such information to the extent that they vary significantly within counties over time. At present, we exclude such variables as few official county-level estimates of this type of information is presently available for 2020 .

${ }^{7}$ There could be positive serial correlation due persistent shocks, such as natural or other disasters, or there could be negative serial correlation due to survivorship bias.

${ }^{8}$ We selected 2011 as the first year in our sample by comparing the predictive performance of our baseline Poisson GLM on 2019 mortality using different starting windows from 2009 to 2015
} 


\subsection{Summary Statistics}

We calculated summary statistics across all counties, metropolitan-nonmetropolitan categories, BEA regions, and BEA regions crossed with metropolitan-nonmetropolitan categories. In line with CDC guidance,[18] we excluded counties with negative excess death rates from the aggregations. In total, 48 county-sets representing less than $2 \%$ of the U.S. population (5.5 million residents) were excluded, yielding a sample size of 1,422 county-sets representing 3,066 counties.

\section{Results}

Across 1,422 county-sets in the U.S. representing 3,066 counties, we estimated that 439,698 excess deaths occurred in 2020. All-cause mortality was higher in most county-sets in 2020 compared to the years 2011 through 2019, leading to substantial excess mortality (Figure 1). The majority of counties also reported excess mortality not assigned to COVID-19. Across all counties, $86.7 \%$ of excess deaths were assigned to COVID-19, revealing that $13.3 \%$ of excess deaths were not assigned to COVID-19.

Table 1 presents summary statistics for excess mortality and excess deaths not assigned to COVID19 across metropolitan-nonmetropolitan categories, BEA Regions, and metropolitan-nonmetropolitan categories crossed with BEA regions. Excess death rates were highest in nonmetro areas (159.9 deaths per 100,000 residents) and large metro areas (148.4 deaths per 100,000 residents) compared to small or medium metro areas (125.8 deaths per 100,000 residents) and large fringe metros (114.5 deaths per 100,000 residents). Across BEA regions, excess mortality was highest in the Mideast (191.5 deaths per 100,000 residents), Great Lakes (146.7 deaths per 100,000 residents), and Southwest (142.9 deaths per 100,000 regions) regions. Figure 2 shows excess death rates in the U.S., highlighting counties with higher excess mortality in darker blue. This figure highlights the geographic dispersion of the COVID-19 pandemic with some areas reporting higher excess mortality than others, such as large central metros in the Mideast (279.9 deaths per 100,000 residents), nonmetro areas in the Southwest (206.0 deaths per 100,000 residents), nonmetro areas in the Southeast (184.5 deaths per 100,000 residents, and large central metros in the Great Lakes (181.7 deaths per 100,000 residents). The figure also identifies specific counties with the highest excess mortality such as Bronx and Queens, New York. Appendix Table A1 presents summary statistics for excess mortality for each state, which further highlights the substantial geographic differences in excess mortality across the country.

Some regions (Mideast, Great Lakes, New England, and Far West) reported more excess deaths in large central metros compared to nonmetro areas. In contrast, the other regions (Southwest, Southeast, Plains, and Rocky Mountains) reported higher excess mortality in nonmetro areas compared to large central metros (Appendix Figure A1). This difference demonstrates that patterns of excess mortality across metropolitan-nonmetropolitan categories varied by BEA region.

There was also significant variation in the proportion of excess deaths assigned to COVID-19 across counties in the U.S., with some counties reporting substantial numbers of excess deaths not assigned to COVID-19. Across the U.S., 91.2 million U.S. residents lived in counties where less than $75 \%$ of excess deaths were assigned to COVID-19 (Appendix Table A2). On average, assignment of excess deaths to COVID-19 was lowest in large central metro areas (79.3\%) compared to medium or small metros (87.4\%), nonmetro areas (89.4\%) and large fringe metros (95.2\%). Regionally, the proportion of excess deaths assigned to COVID-19 was lowest in the Southeast (81.1\%), Far West (81.2\%), Southwest (82.6\%), and Rocky Mountains (85.2\%).

Figure 3 shows the percentage of excess deaths not assigned to COVID-19 across counties in the U.S., labeling counties as having high proportions of unassigned deaths (more than $25 \%$ excess deaths 
not assigned to COVID-19), moderate proportions of unassigned deaths (between 10 and $25 \%$ of excess deaths not assigned to COVID-19), or low proportions of unassigned deaths (between $0 \%$ and $10 \%$ of excess deaths not assigned to COVID-19).

Figure 4 plots excess death rates against directly assigned COVID-19 death rates for each countyset in each BEA region. In these plots, counties above the $45^{\circ}$ line represent county-sets where excess deaths exceeded directly assigned COVID-19 deaths, meaning that excess deaths not assigned to COVID19 occurred in these areas. All regions had some county-sets that reported excess deaths not assigned to COVID-19, and in all regions except New England, the majority of county-sets had excess deaths not assigned to COVID-19. In New England, directly assigned COVID-19 mortality exceeded excess mortality in most counties, indicating a negative excess death rate not assigned to COVID-19. Among these counties in New England, two types of counties emerged: (1) nonmetro counties with low direct COVID-19 death rates where excess death rates were negative, and (2) metro counties with positive excess death rates and substantial numbers of direct COVID-19 deaths that exceeded the number of excess deaths. Appendix Figure A1 shows the proportion of excess deaths assigned to COVID-19 in New England across metropolitan-nonmetropolitan categories. Across large central metros, large fringe metros, and medium or small metros in New England, directly assigned COVID-19 deaths exceeded excess deaths. In nonmetro areas in New England, $67.7 \%$ of excess deaths were assigned to COVID-19, whereas $134.8 \%$ were assigned to COVID-19 in large fringe metro areas, $120.6 \%$ in medium or small metros, and $108.0 \%$ in large central metro areas.

Figure 5 decomposes excess death rates for county-sets with the highest excess death rates not assigned to COVID-19 and the most negative excess death rates not assigned to COVID-19. Most counties across the U.S. experienced substantial numbers of excess deaths not assigned to COVID-19, and many of these excess deaths not assigned to COVID-19 were significantly positive. Counties with large, negative excess mortality not assigned to COVID-19 were mostly in the New England region such as Barnstable/Dukes/Nantucket, Massachusetts, Essex, Massachusetts, Hillsborough, New Hampshire, Bristol/Providence, Rhode Island, and Middlesex, Massachusetts. Other counties outside of New England with statistically significant negative excess death rates not assigned to COVID-19 included Brevard, Florida and Hawaii County, Hawaii.

Appendix Figure A2 and Appendix Figure A3 display a time series of observed death rates from 2011 to 2019 and a comparison of the 2020 predicted death rate and the 2020 expected death rate. In the East South Central region where a relatively large share of excess deaths were not assigned to COVID-19, the observed death rate in 2020 substantially exceeded the expected 2020 death rate and the expected 2020 death rate plus COVID-19 deaths. In New England, however, where direct COVID-19 deaths exceeded excess deaths, the opposite occurred - with the expected death rate plus COVID-19 deaths exceeding the observed death rate in 2020.

Only 48 county-sets, representing less than $2 \%$ of the U.S. population (5.5 million residents) experienced negative excess mortality (where observed mortality was less than expected mortality). Among these counties, no county-sets had statistically significant negative excess death rates where the uncertainty intervals for the excess death rates did not overlap with 0 . This suggests that most counties across the U.S. experienced positive excess mortality in 2020. Appendix Table C1 provides estimates of excess death rates and their uncertainty intervals for 1,470 county-sets across the U.S..

\section{Discussion}

In this study, we produced county-level estimates of excess mortality and the proportion of excess deaths not assigned to COVID-19 and examined geographic variation in mortality across the United 
States. We found that more than one-forth of U.S. residents (91.2 million) lived in counties where less than $75 \%$ of excess deaths were assigned to COVID-19, suggesting that the impact of the COVID-19 pandemic has not been fully registered in many parts of the country. Assignment of excess deaths to COVID-19 was particularly low in the Southeast, Far West, Rocky Mountain, and Southwest regions and in large central metro areas compared to other areas. New England was unique in reporting more directly assigned COVID-19 deaths than excess deaths.

Across counties in the U.S., we estimated that 439,698 total excess deaths occurred in 2020, of which $86.7 \%$ were assigned to COVID-19. This estimate is similar to an estimate of 458,000 excess deaths in the U.S. during 2020 produced by Islam et al.[21] Recent estimates by Woolf et al. calculated 522,368 excess deaths between March 1, 2020, and January 2, 2021, which is higher than our estimate.[4] Our estimate may be more conservative than Woolf et al. because we incorporated historical data from 2011 through 2019 for our calculations of expected mortality whereas Woolf et al. used trends from 2014 through 2019. Using data from 1999 to 2020 and a cubic model, Glei identified 402,743 excess deaths in 2020, which was lower than our estimate.[22] Ahmad et al. found an increase of 503,976 deaths between 2019 and 2020, which is also higher than our estimate of excess mortality.[23] However, this estimate did not control for recent mortality trends.

Our study also reveals substantial heterogeneity in excess deaths and the proportion of excess deaths not assigned to COVID-19 across counties, which are the administrative unit for death registration. This highlights the value of studying excess mortality at the county-level since state and national-estimates mask significant variability within states. This finding is in line with studies of excess deaths in California that found significant differences in excess deaths by race/ethnicity and education, which vary by county. $[24,25]$

Consistent with previous results, we find evidence of wide gaps between excess mortality and directly assigned COVID-19 mortality in many areas across the United States. There are several potential explanations for the discrepancy between excess mortality and directly assigned COVID-19 mortality. One explanation is that the gap reflects underreporting of COVID-19 deaths. Especially early in the pandemic, testing was severely limited, which may have reduced the likelihood of COVID-19 being assigned to the death certificate. Underreporting may have also related to a lack of awareness of the clinical manifestations of COVID-19 early in the pandemic as well as various social, health care, and political factors. $[26,5,14]$ In addition to underreporting, gaps between excess and direct mortality may in part be explained by the indirect effects of the pandemic on mortality levels. Indirect effects could relate to interruptions or delays in health care or the broader social and economic upheaval caused by the pandemic, including loss of employment, social isolation and loneliness, and other factors.[27, 28, 29] Many states have reported increases in overdose deaths during the COVID-19 pandemic, and NCHS data suggest that approximately 19,000 more deaths from unintentional injuries occurred in 2020 than in 2019.[23]

Our study made use of data through December 31, 2020 reported by June 3, 2021, meaning that deaths could be reported for up to five months after they occurred. As a result, our estimate of the proportion of excess deaths not assigned to COVID-19 should be interpreted accordingly, indicating that after accounting for five months of potential reporting and processing delays, $13.3 \%$ of excess deaths were still not assigned to COVID-19. It is important to acknowledge that COVID-19 mortality surveillance data sources such as local health system dashboards that the public uses to interact with county-level data in real-time more substantially underestimate excess mortality due to significant delays in COVID19 reporting that are not accounted for. Similarly, our estimate of the proportion of excess deaths not assigned to COVID-19 (13.3\%) is lower than a previous study using county-level data (17\%), which only incorporated a two and a half month delay between occurrence and reporting. [5]

We also observed a number of counties in which the direct COVID-19 death rate exceeded our estimates 
of excess mortality, especially in New England. These included two types of counties: (1) nonmetro areas that experienced few direct COVID-19 deaths but negative excess mortality and (2) metro areas that had positive excess mortality but where direct COVID-19 deaths exceeded excess deaths. A similar pattern was observed internationally, with countries such as New Zealand and Taiwan having few direct COVID-19 deaths and negative excess mortality while countries such as Luxembourg, France, Belgium, and Costa Rica had positive excess mortality but their direct COVID-19 death rates exceeded their excess death rates.[30] This finding could have occurred for several reasons. First, increases in mortality in 2020 due to COVID-19 may have been offset by declines in deaths from other causes. Provisional data on cause of death for 2020 indicates that flu deaths declined significantly relative to prior years, which may explain part of the offset.[31] Other causes of death may also have experienced reductions in 2020. For example NCHS data indicates that there were approximately 2,600 fewer suicide deaths in 2020 relative to 2019.[23] Shelter-in-place policies may have also been associated with reductions in non-natural deaths. [32] While we did not examine associations with income and education in this study, several of the counties (Barnstable/Dukes/Nantucket, Massachusetts, Bristol/Providence, Rhode Island, and Middlesex, Massachusetts) stood out as economically privileged areas that may have been isolated from the impacts of the pandemic through an ability to work-from-home and avoid household crowding. Another reason direct Covid-19 deaths may have exceeded our estimates of excess deaths is if medical certifiers in a county over-assigned COVID-19 to death certificates. In addition, the differences could at least partially relate to how directly assigned COVID-19 deaths were counted by NCHS; while COVID-19 was listed as the underlying cause in the vast majority of cases, in about $8 \%$ of cases, COVID-19 was listed as a contributing cause and still directly assigned to COVID-19. Finally, frailty selection may have occurred if deaths from COVID-19 occurred among individuals who were likely to die from other causes, resulting in reductions in those causes of death. It is also important to note that in instances where directly assigned COVID-19 deaths exceeded excess deaths in the overall population, patterns could differ among population subgroups related to age, race/ethnicity, and income.

Accurate county-level predictions of excess mortality, and their associated levels of uncertainty, are an important addition to existing work on excess mortality at the state and national level. Countylevel estimates can enable researchers to examine geographic variation in excess mortality and leverage county-level variation in sociodemographic, health, and structural factors to examine inequities in excess deaths. They may also be relevant to local public health departments, to be used in conjunction with their existing efforts to monitor deaths directly assigned to COVID-19. Accurate death tallies at the county-level may play an important role in motivating individual and community responses, including vaccine uptake. Our study indicates that directly assigned COVID-19 death rates have been less accurate measures of excess mortality in areas such as the Southeast and Rocky Mountain regions, which are also areas that are experiencing the slowest vaccine uptake.[33]

This analysis had several limitations. First, unlike prior state-level analyses which leveraged weekly data on deaths, the present study used cumulative data on COVID-19 and all-cause mortality for all of 2020. Given this limitation in the available data, it was not possible to examine changes in excess mortality over time or trends in the proportion of deaths not assigned to COVID-19 at the county level. Examining trends in excess mortality using small-area data is a priority for future research which may help to distinguish the direct effects of the pandemic from indirect consequences associated with interruptions in health care and the social and economic consequences of pandemic response measures. Second, when considering patterns of excess mortality across the United States, an important caveat is that age structure differs across counties. Since COVID-19 mortality is more common in older populations, some of the patterns observed across counties may simply reflect differences in age structure. Thus, an important future direction for county-level analyses of excess mortality is to age standardize the estimates when age- 
specific mortality data become available. Third, the provisional county-level mortality files released by the NCHS did not include information on cause of death, and therefore it was not possible to disentangle the sources of excess deaths in 2020. Decomposing excess deaths by cause of death will be critical to understanding why some counties have a higher fraction of unassigned deaths than others and the extent to which the discrepancies are explained by COVID-19 death undercounts versus indirect pandemic effects. For example, such an analysis might partition natural from non-natural deaths under the assumption that non-natural causes of death are unlikely to represent misasacribed COVID-19 deaths. Information on cause of death will also be valuable for understanding the extent to which declines in mortality from other causes have offset COVID-19 deaths, thereby leading to smaller estimates of both excess deaths and the percent of excess deaths that were not assigned to COVID-19. Given the potential for offset from other causes of death, it is likely our overall finding that $13.3 \%$ of excess deaths were not assigned to COVID-19 represents a lower bound on the percent unassigned. Finally, the data used in the present study are provisional in nature and may be subject to further corrections by the NCHS in the process of generating final death counts by cause of death for 2020 .

In conclusion, the present study builds on prior work by extending estimates of excess mortality and excess deaths not assigned to COVID-19 to US counties. The added geographic detail of these estimates compared to prior studies may facilitate research on the causes and consequences of the COVID19 pandemic on population health and provide useful data for local area health policy and planning. Estimates of excess mortality at the local level can also inform the allocation of resources to areas most impacted by the pandemic and contribute to positive protective behavior feedback loops (i.e. increases in mask-wearing and vaccine uptake). In doing so, they can inform the response to the COVID-19 pandemic and to any future pandemics that the country may face. 
medRxiv preprint doi: https://doi.org/10.1101/2021.04.23.21255564; this version posted August 29, 2021. The copyright holder for this preprint (which was not certified by peer review) is the author/funder, who has granted medRxiv a license to display the preprint in perpetuity.

It is made available under a CC-BY-NC-ND 4.0 International license .

\section{References}

[1] Leon David A, Shkolnikov Vladimir M, Smeeth Liam, Magnus Per, Pechholdová Markéta, Jarvis Christopher I. COVID-19: a need for real-time monitoring of weekly excess deaths Lancet. 2020;395:e81.

[2] Rossen Lauren M, Branum Amy M, Ahmad Farida B, Sutton Paul D, Anderson Robert N. Notes from the Field: Update on Excess Deaths Associated with the COVID-19 Pandemic - United States, January 26, 2020-February 27, 2021 MMWR Morb. Mortal. Wkly. Rep.. 2021;70:570-571.

[3] Weinberger Daniel M, Chen Jenny, Cohen Ted, et al. Estimation of Excess Deaths Associated With the COVID-19 Pandemic in the United States, March to May 2020 JAMA Intern. Med.. 2020;180:1336-1344.

[4] Woolf Steven H, Chapman Derek A, Sabo Roy T, Zimmerman Emily B. Excess Deaths From COVID19 and Other Causes in the US, March 1, 2020, to January 2, 2021 JAMA. 2021.

[5] Stokes Andrew C, Lundberg Dielle J, Elo Irma T, Hempstead Katherine, Bor Jacob, Preston Samuel H. COVID-19 and excess mortality in the United States: A county-level analysis PLoS Med.. 2021;18:e1003571.

[6] Kiang Mathew V, Irizarry Rafael A, Buckee Caroline O, Balsari Satchit. Every Body Counts: Measuring Mortality From the COVID-19 Pandemic Ann. Intern. Med.. 2020;173:1004-1007.

[7] Friedman Ari B, Barfield Deidre, David Guy, et al. Delayed emergencies: The composition and magnitude of non-respiratory emergency department visits during the COVID-19 pandemic $J \mathrm{Am}$ Coll Emerg Physicians Open. 2021;2:e12349.

[8] Hartnett Kathleen P, Kite-Powell Aaron, DeVies Jourdan, et al. Impact of the COVID-19 Pandemic on Emergency Department Visits - United States, January 1, 2019-May 30, 2020 MMWR Morb. Mortal. Wkly. Rep.. 2020;69:699-704.

[9] Matthay Ellicott C, Duchowny Kate A, Riley Alicia R, Galea Sandro. Projected All-Cause Deaths Attributable to COVID-19-Related Unemployment in the United States Am. J. Public Health. 2021;111:696-699.

[10] Faust Jeremy S, Du Chengan, Mayes Katherine Dickerson, et al. Mortality From Drug Overdoses, Homicides, Unintentional Injuries, Motor Vehicle Crashes, and Suicides During the Pandemic, March-August 2020 JAMA. 2021;326:84-86.

[11] Egede Leonard E, Walker Rebekah J. Structural Racism, Social Risk Factors, and Covid-19 - A Dangerous Convergence for Black Americans N. Engl. J. Med.. 2020;383:e77.

[12] Chen Jarvis T, Krieger Nancy. Revealing the Unequal Burden of COVID-19 by Income, Race/Ethnicity, and Household Crowding: US County Versus Zip Code Analyses J. Public Health Manag. Pract.. 2021;27 Suppl 1, COVID-19 and Public Health: Looking Back, Moing Forward:S43$\mathrm{S} 56$.

[13] Tan Shin Bin, deSouza Priyanka, Raifman Matthew. Structural Racism and COVID-19 in the USA: a County-Level Empirical Analysis J Racial Ethn Health Disparities. 2021. 
medRxiv preprint doi: https://doi.org/10.1101/2021.04.23.21255564; this version posted August 29, 2021. The copyright holder for this preprint (which was not certified by peer review) is the author/funder, who has granted medRxiv a license to display the preprint in perpetuity. It is made available under a CC-BY-NC-ND 4.0 International license .

[14] Stokes Andrew C, Lundberg Dielle J, Bor Jacob, Elo Irma T, Hempstead Katherine, Preston Samuel H. Association of Health Care Factors with Excess Deaths Not Assigned to COVID-19 JAMA Network Open; Forthcoming. 2021.

[15] Institute of Medicine . Medicolegal Death Investigation System: Workshop Summary. Washington, DC: The National Academies Press 2003.

[16] Gutierrez Emilio, Rubli Adrian, Tavares Tiago. Information and Behavioral Responses during a Pandemic: Evidence from Delays in COVID-19 Death Reports 2021.

[17] Spencer Merianne R, Ahmad Farida. Timeliness of Death Certificate Data for Mortality Surveillance and Provisional Estimates National Vital Statistics Rapid Release. 2016;001.

[18] National Center for Health Statistics . Technical Notes: Provisional Death Counts for Coronavirus Disease https://www.cdc.gov/nchs/nvss/vsrr/covid19/tech ${ }_{n}$ otes.htm2021. Accessed : $2021-3-29$.

[19] Elo Irma T, Hendi Arun S, Ho Jessica Y, Vierboom Yana C, Preston Samuel H. Trends in NonHispanic White Mortality in the United States by Metropolitan-Nonmetropolitan Status and Region, 1990-2016 Popul. Dev. Rev.. 2019;45:549-583.

[20] McCullagh P, Nelder JA. Binary data in Generalized linear models:98-148Springer 1989.

[21] Islam Nazrul, Shkolnikov Vladimir M, Acosta Rolando J, et al. Excess deaths associated with covid19 pandemic in 2020: age and sex disaggregated time series analysis in 29 high income countries BMJ. 2021;373:n1137.

[22] Glei Dana A. THE US MIDLIFE MORTALITY CRISIS CONTINUES: INCREASED DEATH RATES FROM CAUSES OTHER THAN COVID-19 DURING 2020 medRxiv. 2021.

[23] Ahmad Farida B, Anderson Robert N. The Leading Causes of Death in the US for 2020 JAMA. 2021.

[24] Riley Alicia R, Chen Yea-Hung, Matthay Ellicott C, et al. Excess death among Latino people in California during the COVID-19 pandemic 2021.

[25] Chen Yea-Hung, Glymour M Maria, Catalano Ralph, et al. Excess Mortality in California During the Coronavirus Disease 2019 Pandemic, March to August 2020 JAMA Intern. Med.. 2020.

[26] Boukhris Marouane, Hillani Ali, Moroni Francesco, et al. Cardiovascular Implications of the COVID19 Pandemic: A Global Perspective Can. J. Cardiol.. 2020;36:1068-1080.

[27] Wolfson Julia A, Leung Cindy W. Food Insecurity and COVID-19: Disparities in Early Effects for US Adults Nutrients. 2020;12.

[28] Lange Samantha J, Ritchey Matthew D, Goodman Alyson B, et al. Potential Indirect Effects of the COVID-19 Pandemic on Use of Emergency Departments for Acute Life-Threatening Conditions United States, January-May 20202020.

[29] Wu Bei. Social isolation and loneliness among older adults in the context of COVID-19: a global challenge Glob Health Res Policy. 2020;5:27.

[30] Sanmarchi Francesco, Golinelli Davide, Lenzi Jacopo, et al. Exploring the Gap Between Excess Mortality and COVID-19 Deaths in 67 Countries JAMA Netw Open. 2021;4:e2117359. 
[31] Olsen Sonja J, Azziz-Baumgartner Eduardo, Budd Alicia P, et al. Decreased Influenza Activity During the COVID-19 Pandemic — United States, Australia, Chile, and South Africa, 20202020.

[32] Catalano Ralph, Maria Glymour M, Chen Yea-Hung, Bibbins-Domingo Kirsten. Sheltering in Place and the Likelihood of Nonnatural Death 2021.

[33] U.S. Department of Health \& Human Services . ASPE Predictions of Vaccine Hesitancy for COVID19 Vaccines by Geographic and Sociodemographic Features https://aspe.hhs.gov/pdf-report/vaccinehesitancy 2021. Accessed: 2021-5-1.

[34] Gourieroux Christian, Monfort Alain, Trognon Alain. Pseudo maximum likelihood methods: Theory Econometrica: journal of the Econometric Society. 1984:681-700.

[35] Wooldridge Jeffrey M. Distribution-free estimation of some nonlinear panel data models Journal of Econometrics. 1999;90:77-97.

[36] Cameron A Colin, Trivedi Pravin K. Regression analysis of count data;53. Cambridge university press 2013.

[37] Gelman Andrew, Hill Jennifer. Data analysis using regression and multilevel/hierarchical models. Cambridge university press 2006.

[38] Woolf Steven H, Chapman Derek A, Sabo Roy T, Weinberger Daniel M, Hill Latoya, Taylor Dashaunda D H. Excess Deaths From COVID-19 and Other Causes, March-July 2020 JAMA. 2020;324:1562-1564. 
Table 1: Excess Mortality and Direct COVID-19 Mortality by Metropolitan-Nonmetropolitan Status and BEA Region

\begin{tabular}{|c|c|c|c|c|c|c|c|c|}
\hline Geography & $\begin{array}{r}\text { Expected } \\
\text { Deaths }\end{array}$ & $\begin{array}{r}\text { Observed } \\
\text { Deaths }\end{array}$ & $\begin{array}{l}\text { Observed/ } \\
\text { Expected }\end{array}$ & $\begin{array}{l}\text { Excess } \\
\text { Deaths }\end{array}$ & $\begin{array}{r}\text { Excess } \\
\text { Per } 100,000\end{array}$ & $\begin{array}{r}\text { COVID-19 } \\
\text { Deaths }\end{array}$ & $\begin{array}{l}\text { COVID-19 to } \\
\text { Excess Ratio (\%) }\end{array}$ & $\mathrm{N}$ \\
\hline All & $2,886,647$ & $3,326,345$ & 1.15 & 439,698 & 135.0 & 381,185 & 86.7 & 1,422 \\
\hline \multicolumn{9}{|c|}{ By Metro-Nonmetro Status } \\
\hline Lg central metro & 742,272 & 892,036 & 1.20 & 149,764 & 148.4 & 118,797 & 79.3 & 66 \\
\hline Lg fringe metro & 682,033 & 776,535 & 1.14 & 94,502 & 114.5 & 89,988 & 95.2 & 265 \\
\hline $\mathrm{Md} / \mathrm{Sm}$ metro & 892,436 & $1,010,012$ & 1.13 & 117,576 & 125.8 & 102,775 & 87.4 & 459 \\
\hline Nonmetro & 569,906 & 647,762 & 1.14 & 77,856 & 159.9 & 69,625 & 89.4 & 632 \\
\hline \multicolumn{9}{|l|}{ By BEA Region } \\
\hline Far West & 397,361 & 449,298 & 1.13 & 51,937 & 94.5 & 42,185 & 81.2 & 90 \\
\hline Great Lakes & 462,582 & 531,237 & 1.15 & 68,655 & 146.7 & 60,122 & 87.6 & 249 \\
\hline Mideast & 432,817 & 525,272 & 1.21 & 92,455 & 191.5 & 83,205 & 90.0 & 139 \\
\hline New England & 123,438 & 139,135 & 1.13 & 15,697 & 115.2 & 19,235 & 122.5 & 38 \\
\hline Plains & 202,289 & 230,952 & 1.14 & 28,663 & 133.5 & 27,257 & 95.1 & 171 \\
\hline Rocky Mountain & 89,391 & 100,752 & 1.13 & 11,361 & 91.7 & 9,679 & 85.2 & 65 \\
\hline Southeast & 842,531 & 952,109 & 1.13 & 109,578 & 128.6 & 88,835 & 81.1 & 512 \\
\hline Southwest & 336,239 & 397,590 & 1.18 & 61,351 & 142.9 & 50,667 & 82.6 & 158 \\
\hline \multicolumn{9}{|l|}{ Far West } \\
\hline Lg central metro & 204,753 & 237,522 & 1.16 & 32,769 & 106.6 & 26,042 & 79.5 & 11 \\
\hline $\mathrm{Lg}$ fringe metro & 63,188 & 69,674 & 1.10 & 6,486 & 74.2 & 5,444 & 83.9 & 14 \\
\hline Md/Sm metro & 104,592 & 115,881 & 1.11 & 11,289 & 85.8 & 9,473 & 83.9 & 41 \\
\hline Nonmetro & 24,828 & 26,221 & 1.06 & 1,393 & 60.6 & 1,226 & 88.0 & 24 \\
\hline \multicolumn{9}{|l|}{ Great Lakes } \\
\hline Lg central metro & 115,547 & 138,880 & 1.20 & 23,333 & 181.7 & 17,988 & 77.1 & 8 \\
\hline $\mathrm{Lg}$ fringe metro & 110,574 & 126,187 & 1.14 & 15,613 & 125.1 & 14,084 & 90.2 & 50 \\
\hline Md/Sm metro & 129,980 & 146,656 & 1.13 & 16,676 & 133.2 & 15,300 & 91.7 & 73 \\
\hline Nonmetro & 106,481 & 119,514 & 1.12 & 13,033 & 145.7 & 12,750 & 97.8 & 118 \\
\hline \multicolumn{9}{|l|}{ Mideast } \\
\hline Lg central metro & 124,426 & 169,593 & 1.36 & 45,167 & 279.9 & 35,932 & 79.6 & 14 \\
\hline $\mathrm{Lg}$ fringe metro & 179,932 & 212,145 & 1.18 & 32,213 & 158.6 & 32,515 & 100.9 & 46 \\
\hline Md/Sm metro & 97,065 & 108,968 & 1.12 & 11,903 & 130.2 & 11,843 & 99.5 & 48 \\
\hline Nonmetro & 31,394 & 34,566 & 1.10 & 3,172 & 118.4 & 2,915 & 91.9 & 31 \\
\hline \multicolumn{9}{|l|}{ New England } \\
\hline Lg central metro & 13,156 & 15,962 & 1.21 & 2,806 & 164.3 & 3,031 & 108.0 & 2 \\
\hline $\mathrm{Lg}$ fringe metro & 52,243 & 58,707 & 1.12 & 6,464 & 109.1 & 8,717 & 134.8 & 12 \\
\hline Md/Sm metro & 43,711 & 49,280 & 1.13 & 5,569 & 118.0 & 6,716 & 120.6 & 12 \\
\hline Nonmetro & 14,328 & 15,186 & 1.06 & 858 & 67.7 & 771 & 89.9 & 12 \\
\hline \multicolumn{9}{|l|}{ Plains } \\
\hline Lg central metro & 23,236 & 27,015 & 1.16 & 3,779 & 133.1 & 3,097 & 82.0 & 4 \\
\hline $\mathrm{Lg}$ fringe metro & 37,735 & 42,207 & 1.12 & 4,472 & 99.2 & 4,545 & 101.6 & 20 \\
\hline Md/Sm metro & 51,855 & 59,229 & 1.14 & 7,374 & 121.1 & 6,638 & 90.0 & 37 \\
\hline Nonmetro & 89,463 & 102,501 & 1.15 & 13,038 & 162.4 & 12,977 & 99.5 & 110 \\
\hline \multicolumn{9}{|l|}{ Rocky Mountain } \\
\hline Lg central metro & 11,820 & 13,645 & 1.15 & 1,825 & 95.0 & 1,399 & 76.6 & 2 \\
\hline $\mathrm{Lg}$ fringe metro & 14,487 & 16,901 & 1.17 & 2,414 & 104.9 & 1,934 & 80.1 & 6 \\
\hline Md/Sm metro & 38,962 & 43,044 & 1.10 & 4,082 & 75.0 & 3,828 & 93.8 & 22 \\
\hline Nonmetro & 24,122 & 27,162 & 1.13 & 3,040 & 111.9 & 2,518 & 82.8 & 35 \\
\hline \multicolumn{9}{|l|}{ Southeast } \\
\hline Lg central metro & 122,205 & 140,196 & 1.15 & 17,991 & 117.3 & 14,008 & 77.9 & 17 \\
\hline Lg fringe metro & 176,903 & 198,166 & 1.12 & 21,263 & 99.0 & 17,937 & 84.4 & 92 \\
\hline $\mathrm{Md} / \mathrm{Sm}$ metro & 337,046 & 377,079 & 1.12 & 40,033 & 125.2 & 32,233 & 80.5 & 183 \\
\hline Nonmetro & 206,377 & 236,668 & 1.15 & 30,291 & 184.5 & 24,657 & 81.4 & 220 \\
\hline \multicolumn{9}{|l|}{ Southwest } \\
\hline Lg central metro & 127,130 & 149,223 & 1.17 & 22,093 & 113.9 & 17,300 & 78.3 & 8 \\
\hline Lg fringe metro & 46,973 & 52,548 & 1.12 & 5,575 & 81.8 & 4,812 & 86.3 & 25 \\
\hline Md/Sm metro & 89,224 & 109,875 & 1.23 & 20,651 & 198.6 & 16,744 & 81.1 & 43 \\
\hline Nonmetro & 72,912 & 85,944 & 1.18 & 13,032 & 206.0 & 11,811 & 90.6 & 82 \\
\hline
\end{tabular}

Notes: Aggregated results by various geographic regions. Aggregate rates are computed by summing actual and predicted counts over counties in a particular region and dividing by the summed population. Note that this is equivalent to the population-weighted means of the county-level rates. COVID-19 deaths refer to deaths that appeared as either an underlying or contributing cause on the death certificate. In line with CDC guidance,[18] we exclude counties with negative excess death rates from the aggregations. 48 county-sets representing less than $2 \%$ of the U.S. population (5.5 million residents) were excluded. 
Figure 1: Distribution of All-Cause Deaths, Excess Deaths, and Excess Deaths Excluding COVID-19 Deaths per 100,000 Person-Years across U.S. Counties

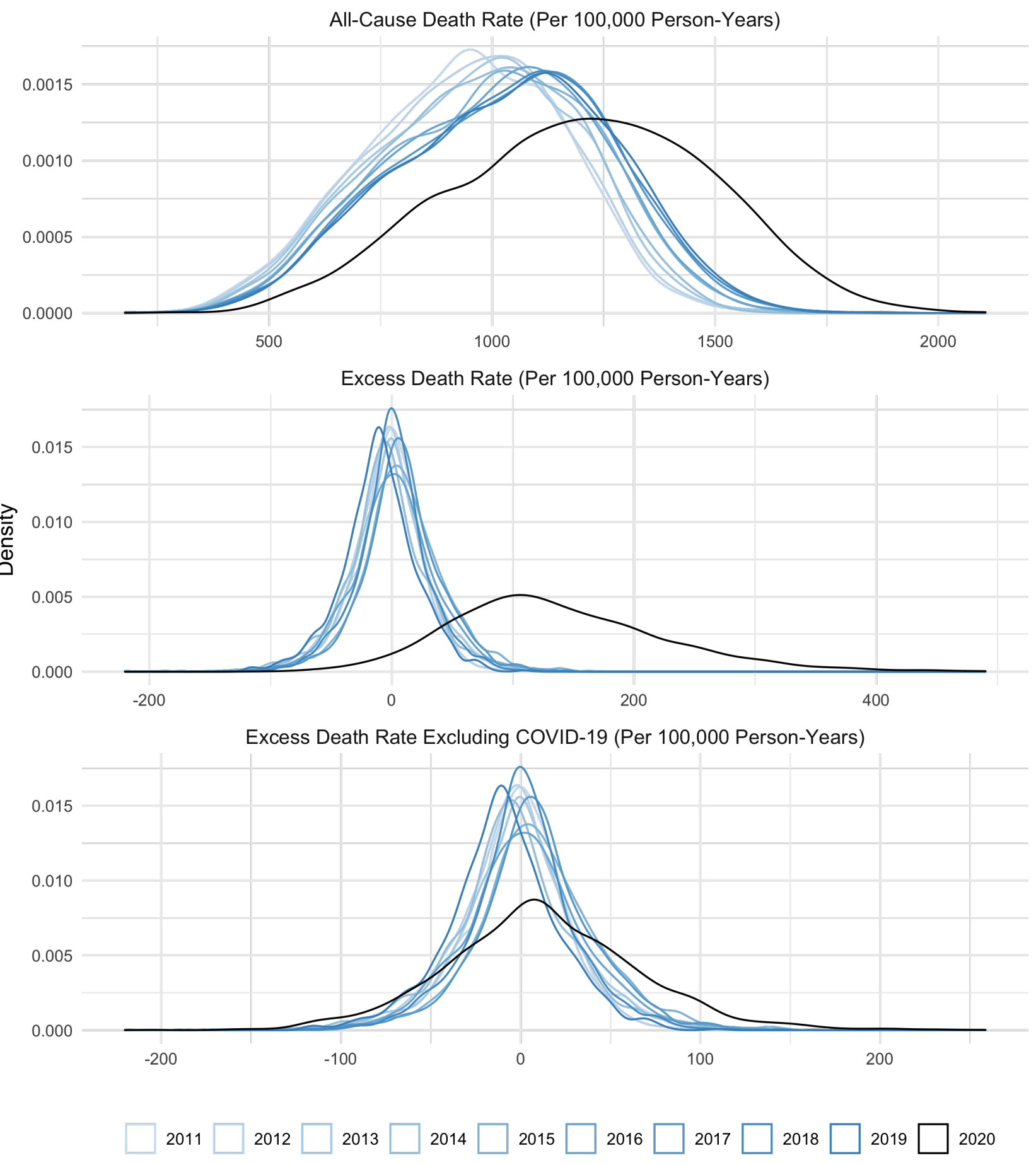

Notes: Expected deaths are computed as the in-sample fitted value for years 2011-2019. For 2020, expected deaths are computed as the out-of-sample fitted value. 
medRxiv preprint doi: https://doi.org/10.1101/2021.04.23.21255564; this version posted August 29, 2021. The copyright holder for this preprint (which was not certified by peer review) is the author/funder, who has granted medRxiv a license to display the preprint in perpetuity.

It is made available under a CC-BY-NC-ND 4.0 International license .

Figure 2: Excess Mortality Rates across U.S. Counties
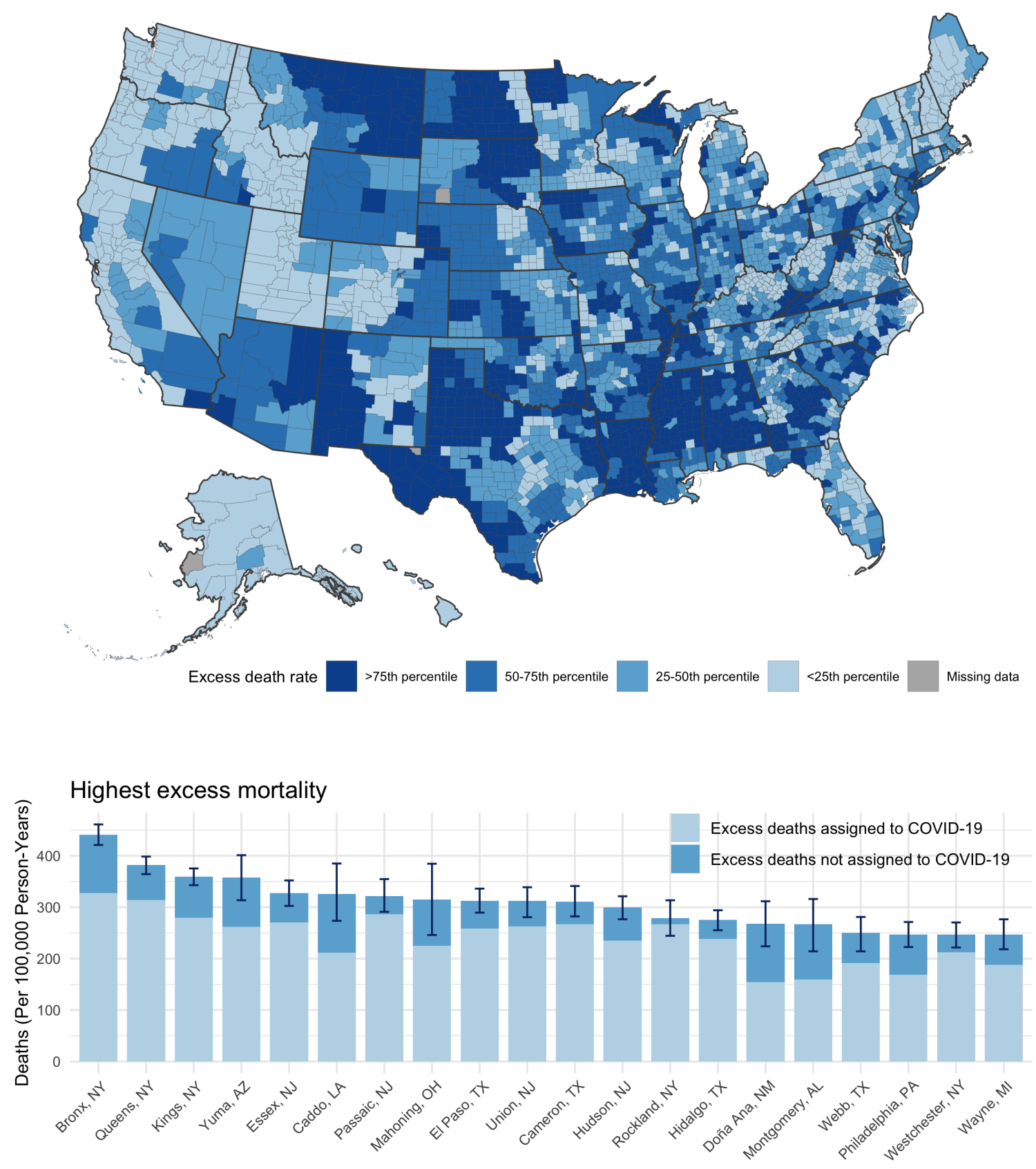

Notes: (top) U.S. counties labeled according to excess death rates, with the largest excess death rates in dark blue. (bottom) Counties with the highest excess mortality in the U.S. decomposed into directly assigned COVID-19 death rates and excess death rates not assigned to COVID-19. Limited to counties with populations of 200,000 residents or greater. 
medRxiv preprint doi: https://doi.org/10.1101/2021.04.23.21255564; this version posted August 29, 2021. The copyright holder for this preprint (which was not certified by peer review) is the author/funder, who has granted medRxiv a license to display the preprint in perpetuity.

It is made available under a CC-BY-NC-ND 4.0 International license .

Figure 3: Proportion of Excess Deaths Not Assigned to COVID-19 across U.S. Counties
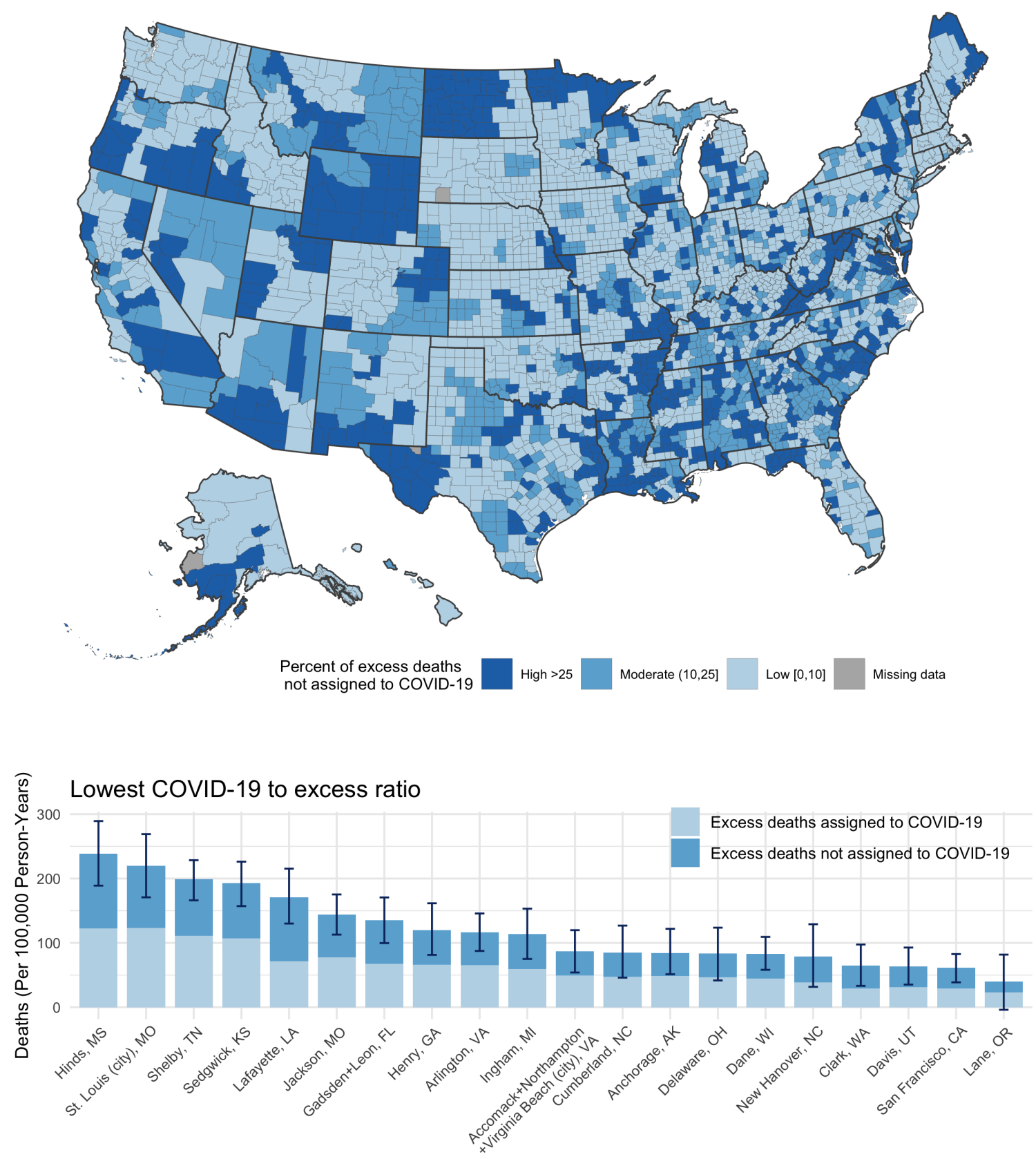

Notes: (top) U.S. counties labeled according to the proportion of excess deaths not assigned to COVID-19, with the highest proportion of unassigned deaths in dark blue. (bottom) Counties with the highest proportion of unassigned deaths decomposed into directly assigned COVID-19 death rates and excess death rates not assigned to COVID-19. Limited to counties with populations of 200,000 residents or greater. 
medRxiv preprint doi: https://doi.org/10.1101/2021.04.23.21255564; this version posted August 29, 2021. The copyright holder for this preprint (which was not certified by peer review) is the author/funder, who has granted medRxiv a license to display the preprint in perpetuity.

It is made available under a CC-BY-NC-ND 4.0 International license .

Figure 4: Plots of Excess Death Rates vs. Direct COVID-19 Death Rates by BEA Region
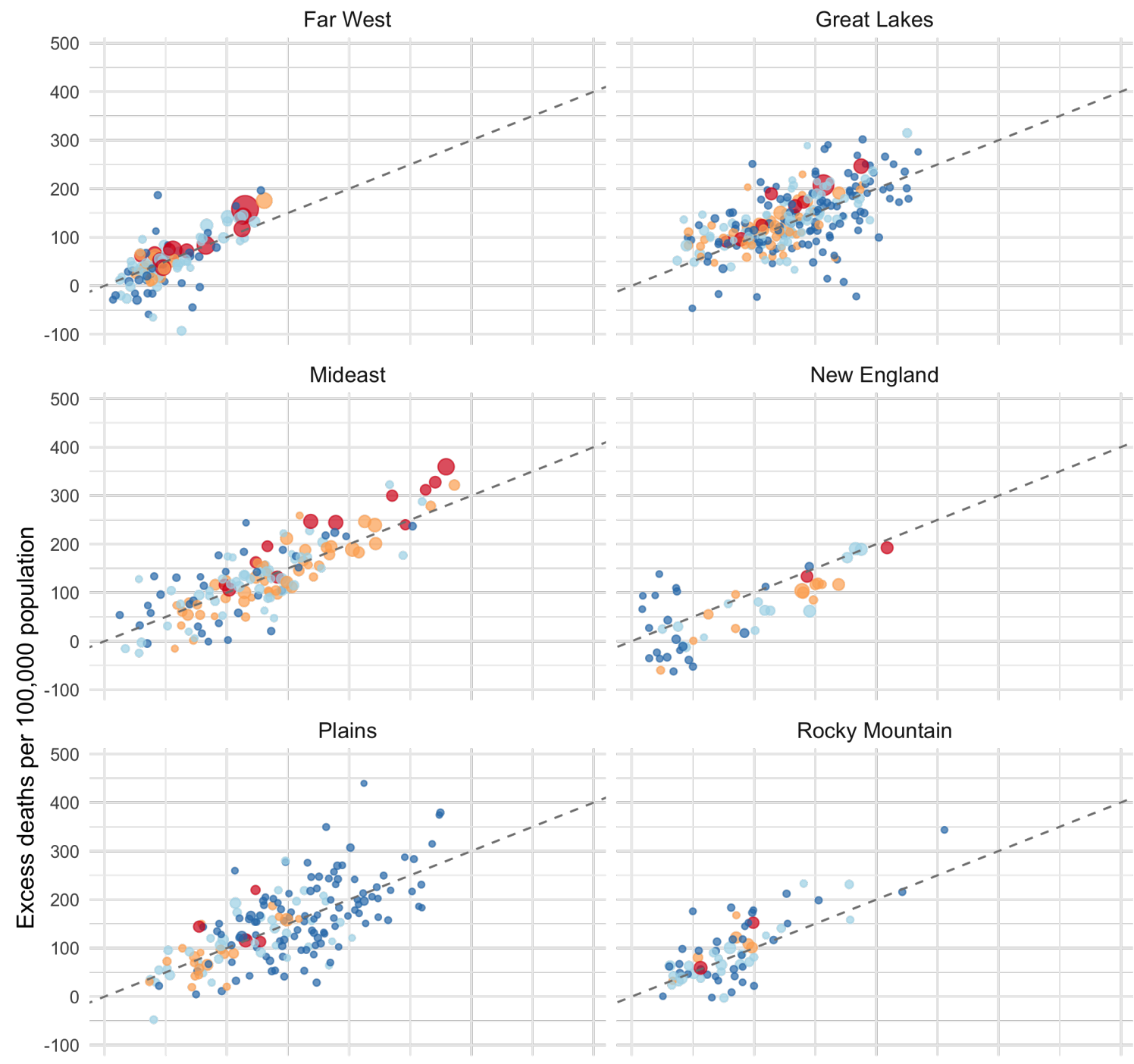

New England

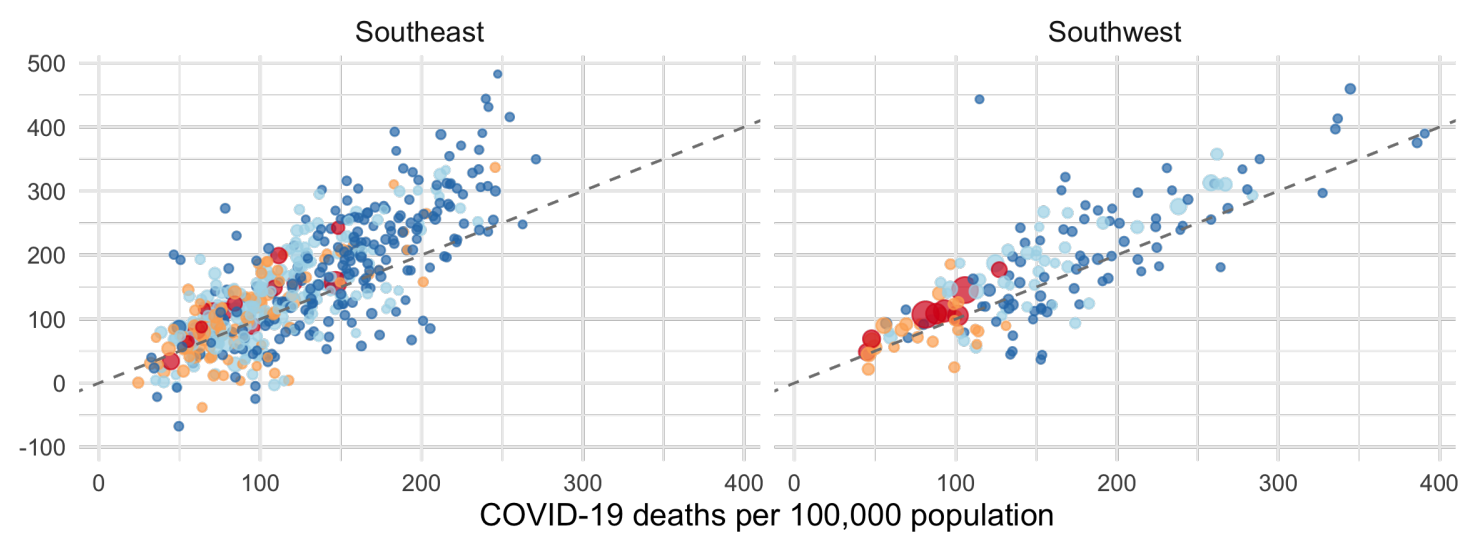

- Lg central metro $\bullet \mathrm{Lg}$ fringe metro $\bullet \mathrm{Md} / \mathrm{Sm}$ metro $\bullet \quad$ Nonmetro

Notes: Counties above the $45^{\circ}$ line represent areas where excess deaths exceeded direct COVID-19 deaths, meaning the excess death rate not assigned to COVID-19 was positive. Counties below the $45^{\circ}$ line represent areas where direct COVID19 deaths exceeded excess deaths, meaning that the excess 1 path rate not assigned to COVID-19 was negative. Excluded counties with direct COVID-19 death rates or excess death rates that were above the 99th percentile for each BEA region. 
Figure 5: U.S. Counties with the Highest Excess Death Rates Not Assigned to COVID-19 and Statistically Significant Negative Excess Death Rates Not Assigned to COVID-19

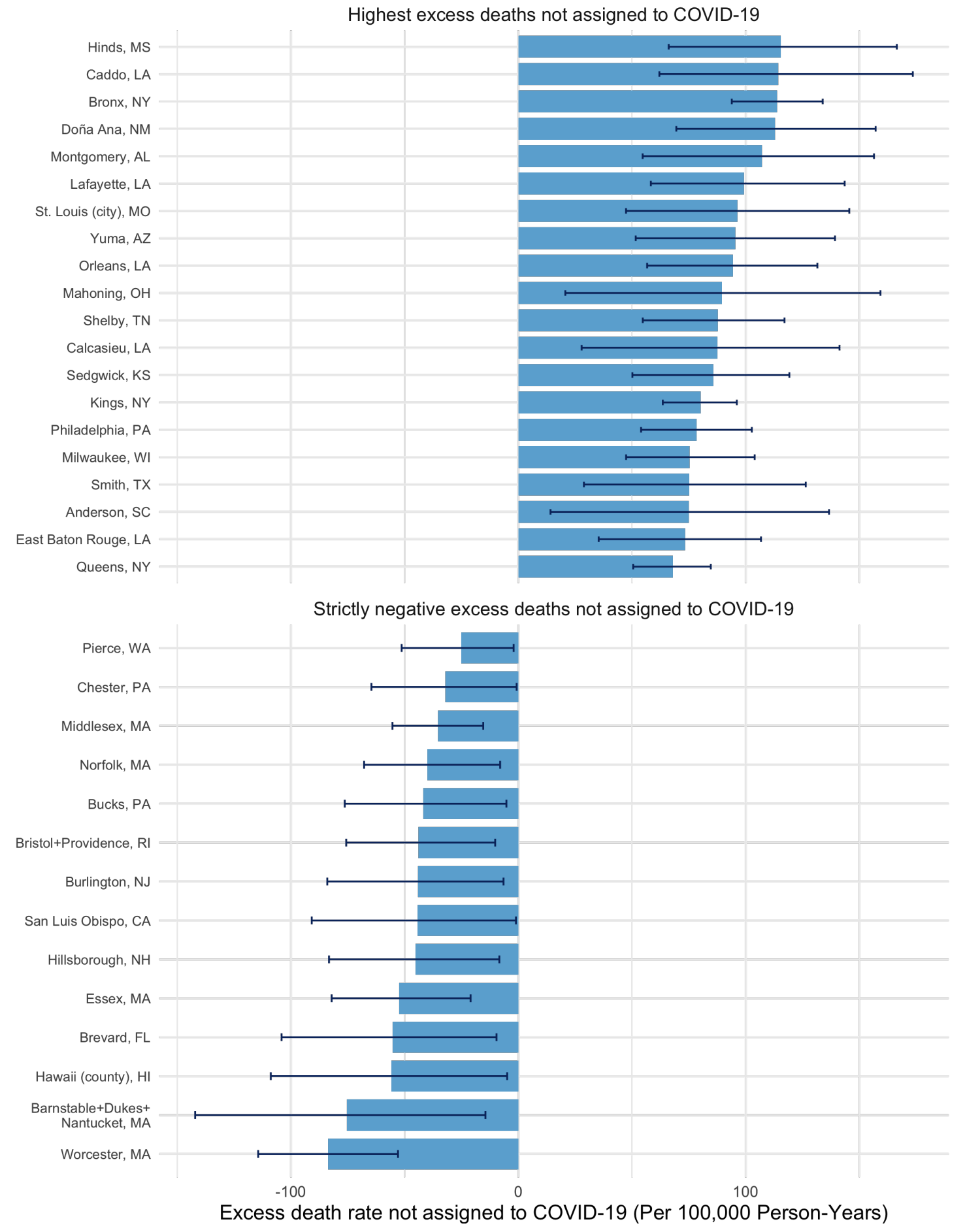

Notes: (top) U.S. county-sets with the highest, statistically significant positive excess death rates not assigned to COVID19. (bottom) U.S. county-sets with statistically significant negative excess death rates not assigned to COVID-19. The 95\% confidence intervals indicate uncertainty in the excess death estimates as calculated by the bootstrapping procedure. Limited to counties with populations of 200,000 residents or greater. 
medRxiv preprint doi: https://doi.org/10.1101/2021.04.23.21255564; this version posted August 29, 2021. The copyright holder for this preprint (which was not certified by peer review) is the author/funder, who has granted medRxiv a license to display the preprint in perpetuity.

It is made available under a CC-BY-NC-ND 4.0 International license .

\section{Appendix A: Tables and Figures}

Figure A1: Excess Death Rates and Proportion of Excess Deaths Assigned to COVID-19 by BEA Region and Metropolitan-Nonmetropolitan Status

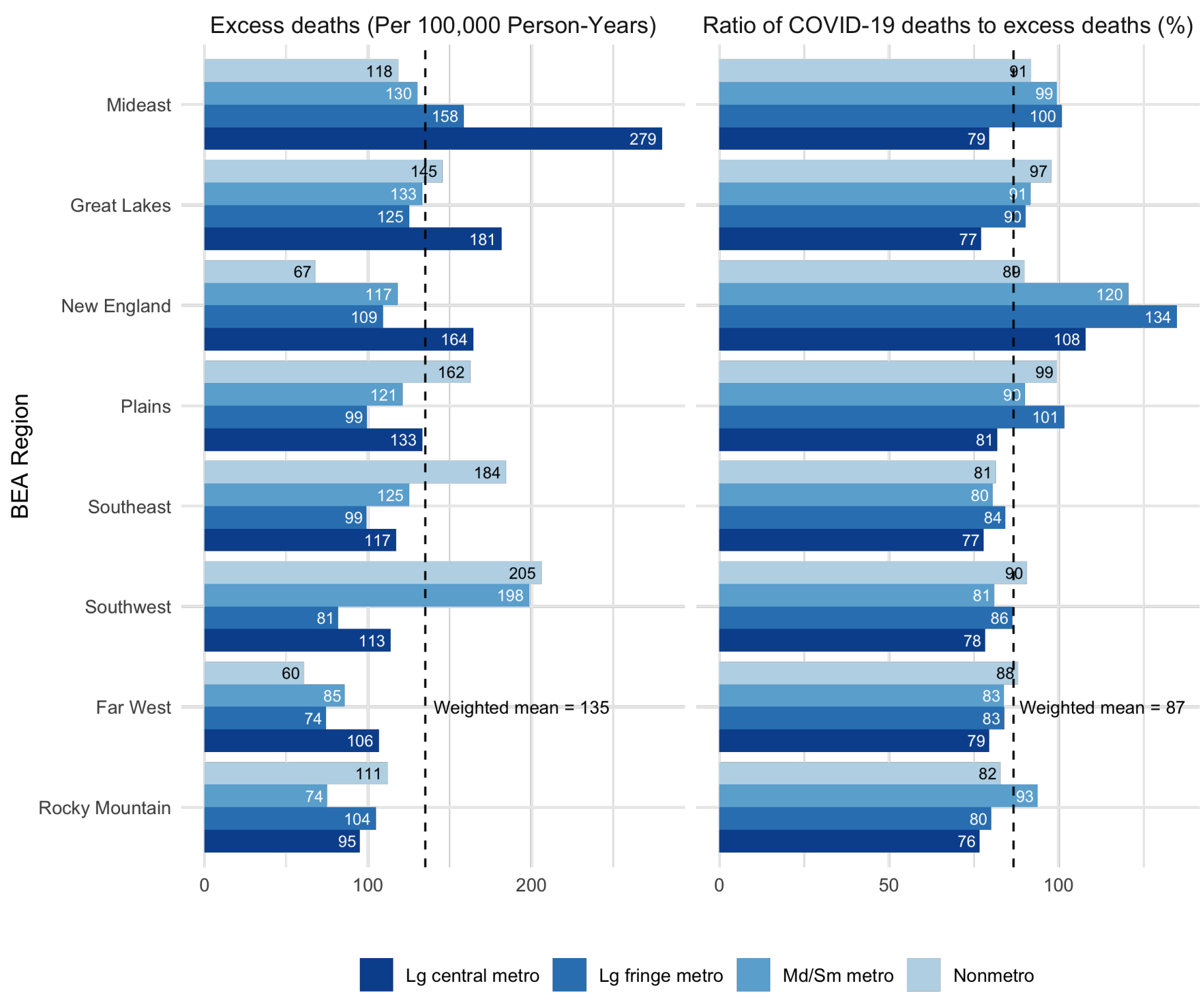

Notes: Aggregated results by various geographic regions. Aggregate rates are computed by summing actual and predicted counts over counties in a particular region and dividing by the summed population. Note that this is equivalent to the population-weighted means of the county-level rates. COVID-19 deaths refer to deaths that appeared as either an underlying or contributing cause on the death certificate. In line with CDC guidance,[18] 48 county-sets with negative excess mortality representing less than $2 \%$ of the U.S. population (5.5 million residents) were excluded. 
Figure A2: Comparison of Observed and Expected Deaths in Large Counties, 2011-2020
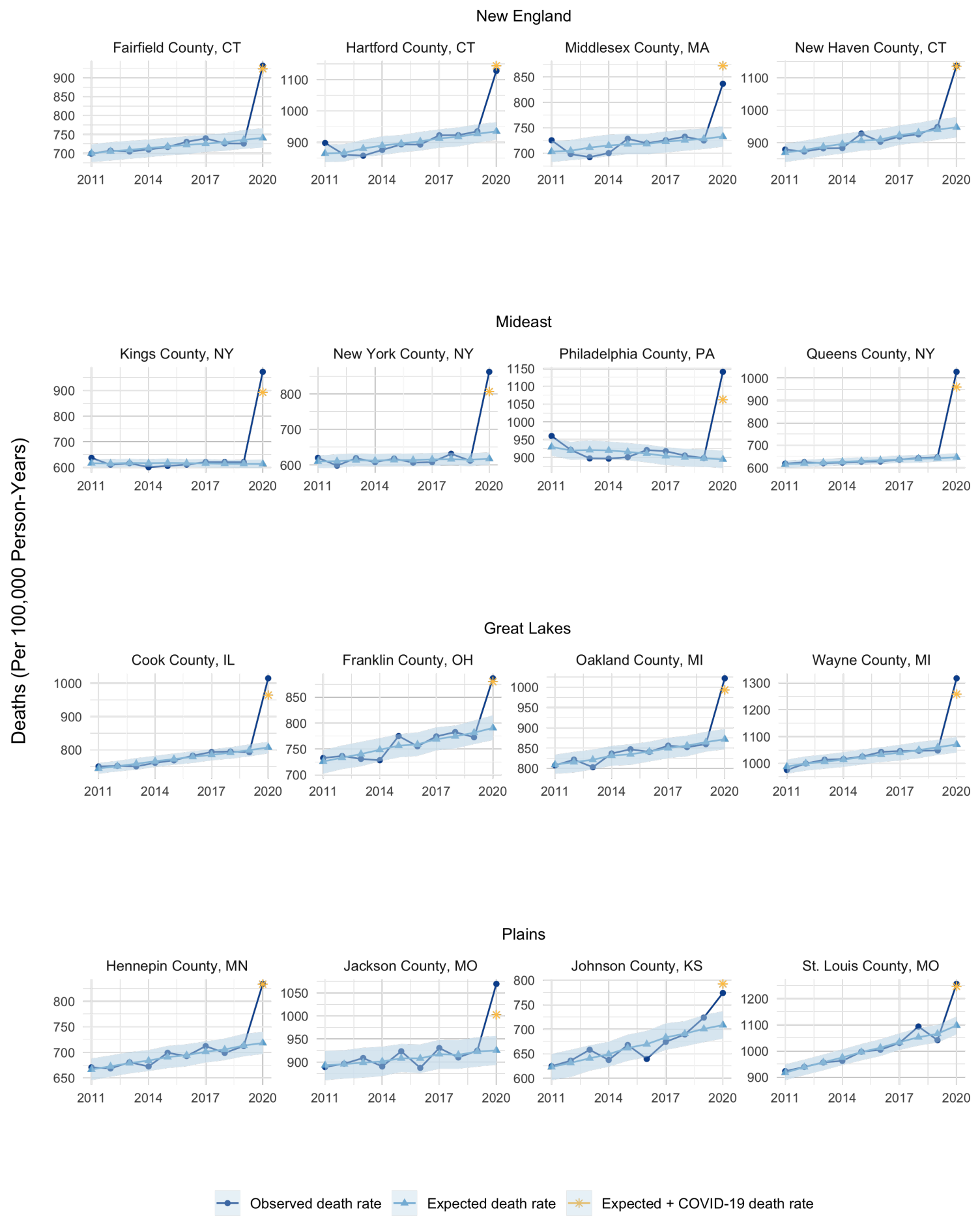

Notes: Observed and expected deaths for largest counties by population in each BEA Region from 2011-2020. Expected death counts are generated using a Poisson GLM with data from 2011-2019. The shaded area represents a 95\% prediction region for each point. These regions are generated using a parametric bootstrap. 
Figure A3: Comparison of Observed and Expected Deaths in Large Counties, 2011-2020
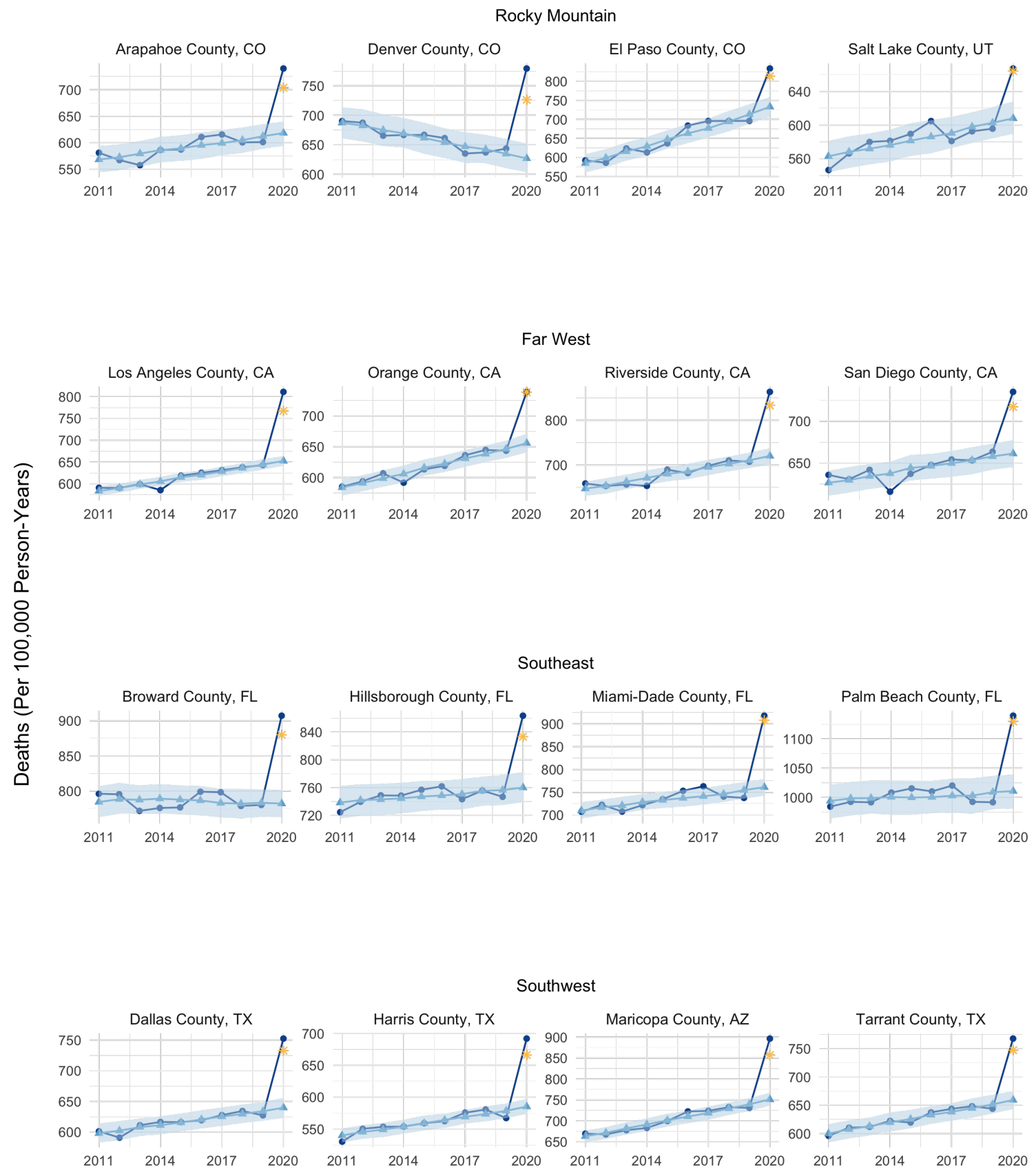

$\rightarrow$ Observed death rate $\rightarrow$ Expected death rate $\rightarrow$ Expected + COVID-19 death rate

Notes: Observed and expected deaths for largest counties by population in each BEA Region from 2011-2020. Expected death counts are generated using a Poisson GLM with data from 2011-2019. The shaded area represents a 95\% prediction region for each point. These regions are generated using a parametric bootstrap. 
Table A1: Excess Mortality and COVID-19 Mortality by State

\begin{tabular}{|c|c|c|c|c|c|c|c|c|}
\hline State & $\begin{array}{r}\text { Expected } \\
\text { Deaths }\end{array}$ & $\begin{array}{r}\text { Observed } \\
\text { Deaths }\end{array}$ & $\begin{array}{c}\text { Observed/ } \\
\text { Expected }\end{array}$ & $\begin{array}{l}\text { Excess } \\
\text { Deaths }\end{array}$ & $\begin{array}{r}\text { Excess } \\
\text { Per } 100,000\end{array}$ & $\begin{array}{r}\text { COVID-19 } \\
\text { Deaths }\end{array}$ & $\begin{array}{c}\text { COVID-19 to } \\
\text { Excess Ratio }(\%)\end{array}$ & $\mathrm{N}$ \\
\hline $\mathrm{AK}$ & 4,181 & 4,630 & 1.11 & 449 & 67.4 & 269 & 59.9 & 6 \\
\hline $\mathrm{AL}$ & 55,639 & 64,776 & 1.16 & 9,137 & 186.0 & 6,991 & 76.5 & 40 \\
\hline $\mathrm{AR}$ & 33,610 & 38,099 & 1.13 & 4,489 & 147.9 & 4,014 & 89.4 & 32 \\
\hline $\mathrm{AZ}$ & 63,304 & 75,741 & 1.20 & 12,437 & 167.8 & 9,182 & 73.8 & 11 \\
\hline $\mathrm{CA}$ & 269,553 & 312,029 & 1.16 & 42,476 & 108.7 & 33,004 & 77.7 & 41 \\
\hline $\mathrm{CO}$ & 40,525 & 46,516 & 1.15 & 5,991 & 103.4 & 4,926 & 82.2 & 23 \\
\hline $\mathrm{CT}$ & 31,986 & 37,831 & 1.18 & 5,845 & 164.0 & 6,214 & 106.3 & 8 \\
\hline DC & 5,035 & 6,198 & 1.23 & 1,163 & 162.5 & 885 & 76.1 & 1 \\
\hline $\mathrm{DE}$ & 9,868 & 11,071 & 1.12 & 1,203 & 121.8 & 1,038 & 86.3 & 3 \\
\hline $\mathrm{FL}$ & 214,336 & 237,542 & 1.11 & 23,206 & 106.7 & 21,324 & 91.9 & 48 \\
\hline GA & 89,034 & 102,400 & 1.15 & 13,366 & 125.4 & 10,257 & 76.7 & 67 \\
\hline $\mathrm{HI}$ & 9,204 & 9,580 & 1.04 & 376 & 33.1 & 321 & 85.3 & 2 \\
\hline IA & 30,695 & 35,413 & 1.15 & 4,718 & 153.1 & 4,725 & 100.2 & 31 \\
\hline ID & 13,512 & 14,740 & 1.09 & 1,228 & 74.1 & 1,393 & 113.5 & 13 \\
\hline IL & 111,763 & 132,652 & 1.19 & 20,889 & 165.1 & 17,268 & 82.7 & 51 \\
\hline IN & 68,213 & 78,017 & 1.14 & 9,804 & 145.2 & 9,337 & 95.2 & 50 \\
\hline KS & 28,040 & 32,045 & 1.14 & 4,005 & 137.5 & 3,588 & 89.6 & 25 \\
\hline $\mathrm{KY}$ & 50,114 & 55,041 & 1.10 & 4,927 & 110.9 & 4,518 & 91.7 & 44 \\
\hline LA & 46,735 & 56,741 & 1.21 & 10,006 & 215.7 & 7,081 & 70.8 & 35 \\
\hline MA & 60,873 & 68,213 & 1.12 & 7,340 & 105.3 & 10,103 & 137.6 & 12 \\
\hline $\mathrm{MD}$ & 52,411 & 60,073 & 1.15 & 7,662 & 126.1 & 6,706 & 87.5 & 19 \\
\hline $\mathrm{ME}$ & 9,162 & 9,527 & 1.04 & 365 & 45.1 & 256 & 70.2 & 6 \\
\hline MI & 100,676 & 115,750 & 1.15 & 15,074 & 152.0 & 12,382 & 82.1 & 49 \\
\hline $\mathrm{MN}$ & 46,670 & 52,370 & 1.12 & 5,700 & 100.0 & 5,932 & 104.1 & 34 \\
\hline $\mathrm{MO}$ & 64,756 & 73,887 & 1.14 & 9,131 & 148.2 & 7,774 & 85.1 & 45 \\
\hline MS & 33,562 & 40,176 & 1.20 & 6,614 & 221.9 & 5,023 & 75.9 & 36 \\
\hline MT & 10,594 & 12,022 & 1.13 & 1,428 & 132.1 & 1,274 & 89.2 & 11 \\
\hline $\mathrm{NC}$ & 97,101 & 106,818 & 1.10 & 9,717 & 93.3 & 8,540 & 87.9 & 61 \\
\hline ND & 6,497 & 8,054 & 1.24 & 1,557 & 202.0 & 1,337 & 85.8 & 8 \\
\hline $\mathrm{NE}$ & 17,395 & 19,540 & 1.12 & 2,145 & 109.8 & 2,306 & 107.5 & 18 \\
\hline NH & 6,489 & 6,928 & 1.07 & 439 & 60.0 & 652 & 148.5 & 2 \\
\hline NJ & 76,860 & 95,965 & 1.25 & 19,105 & 213.6 & 17,927 & 93.8 & 21 \\
\hline $\mathrm{NM}$ & 19,943 & 23,749 & 1.19 & 3,806 & 181.3 & 3,012 & 79.1 & 17 \\
\hline NV & 26,962 & 30,617 & 1.14 & 3,655 & 115.6 & 3,462 & 94.7 & 5 \\
\hline NY & 151,125 & 196,520 & 1.30 & 45,395 & 242.5 & 38,404 & 84.6 & 45 \\
\hline $\mathrm{OH}$ & 127,267 & 142,815 & 1.12 & 15,548 & 133.2 & 14,930 & 96.0 & 61 \\
\hline OK & 41,600 & 47,795 & 1.15 & 6,195 & 156.3 & 5,548 & 89.6 & 33 \\
\hline OR & 36,631 & 38,587 & 1.05 & 1,956 & 48.0 & 1,626 & 83.1 & 18 \\
\hline PA & 137,517 & 155,445 & 1.13 & 17,928 & 139.8 & 18,245 & 101.8 & 50 \\
\hline RI & 10,342 & 11,819 & 1.14 & 1,477 & 139.5 & 1,869 & 126.5 & 4 \\
\hline $\mathrm{SC}$ & 53,223 & 61,179 & 1.15 & 7,956 & 152.6 & 5,782 & 72.7 & 30 \\
\hline SD & 8,236 & 9,643 & 1.17 & 1,407 & 158.8 & 1,595 & 113.4 & 10 \\
\hline $\mathrm{TN}$ & 74,695 & 84,197 & 1.13 & 9,502 & 137.8 & 7,452 & 78.4 & 46 \\
\hline TX & 211,391 & 250,305 & 1.18 & 38,914 & 132.1 & 32,925 & 84.6 & 97 \\
\hline UT & 19,669 & 21,491 & 1.09 & 1,822 & 55.6 & 1,560 & 85.6 & 11 \\
\hline VA & 71,529 & 79,893 & 1.12 & 8,364 & 97.9 & 6,252 & 74.7 & 52 \\
\hline VT & 4,585 & 4,817 & 1.05 & 232 & 47.8 & 141 & 60.8 & 6 \\
\hline WA & 50,831 & 53,855 & 1.06 & 3,024 & 44.4 & 3,503 & 115.8 & 18 \\
\hline WI & 54,662 & 62,003 & 1.13 & 7,341 & 126.7 & 6,205 & 84.5 & 38 \\
\hline WV & 22,954 & 25,247 & 1.10 & 2,293 & 132.7 & 1,601 & 69.8 & 21 \\
\hline WY & 5,091 & 5,983 & 1.18 & 892 & 155.1 & 526 & 58.9 & 7 \\
\hline
\end{tabular}

Notes: Aggregated results by various geographic regions. Aggregate rates are computed by summing actual and predicted counts over counties in a particular region and dividing by the summed population. Note that this is equivalent to the population-weighted means of the county-level rates. COVID-19 deaths refer to deaths that appeared as either an underlying or contributing cause on the death certificate. In line with CDC guidance,[18] we exclude counties with negative excess death rates from the aggregations. 48 county-sets representing less than $2 \%$ of the U.S. population (5.5 million residents) were excluded. 
Table A2: U.S. Population Living in Counties with Different Proportions of Excess Deaths Assigned to COVID-19

\begin{tabular}{crrrr}
\hline $\begin{array}{c}\text { Percent of excess deaths } \\
\text { assigned to COVID-19 }\end{array}$ & $\begin{array}{r}\text { Number of } \\
\text { county sets }\end{array}$ & Population & $\begin{array}{r}\text { Cumulative } \\
\text { population }\end{array}$ & $\begin{array}{r}\text { Cumulative } \\
\text { percent }\end{array}$ \\
\hline$(5,10]$ & 1 & 55,379 & 55,379 & $0.02 \%$ \\
$(10,15]$ & 1 & 58,214 & 113,593 & $0.03 \%$ \\
$(15,20]$ & 1 & 57,834 & 171,427 & $0.05 \%$ \\
$(20,25]$ & 5 & 341,482 & 512,909 & $0.15 \%$ \\
$(25,30]$ & 4 & 247,713 & 760,622 & $0.23 \%$ \\
$(30,35]$ & 5 & 422,780 & $1,183,402$ & $0.36 \%$ \\
$(35,40]$ & 6 & 528,800 & $1,712,202$ & $0.52 \%$ \\
$(40,45]$ & 12 & $1,735,533$ & $3,447,735$ & $1.04 \%$ \\
$(45,50]$ & 22 & $3,224,932$ & $6,672,667$ & $2.02 \%$ \\
$(50,55]$ & 45 & $5,546,759$ & $12,219,426$ & $3.69 \%$ \\
$(55,60]$ & 54 & $8,418,387$ & $20,637,813$ & $6.23 \%$ \\
$(60,65]$ & 77 & $14,181,616$ & $34,819,429$ & $10.52 \%$ \\
$(65,70]$ & 84 & $19,064,559$ & $53,883,988$ & $16.27 \%$ \\
$(70,75]$ & 93 & $37,334,323$ & $91,218,311$ & $27.55 \%$ \\
$(75,80]$ & 114 & $43,347,704$ & $134,566,015$ & $40.64 \%$ \\
$(80,85]$ & 98 & $31,868,535$ & $166,434,550$ & $50.26 \%$ \\
$(85,90]$ & 88 & $21,619,839$ & $188,054,389$ & $56.79 \%$ \\
$(90,95]$ & 84 & $23,402,967$ & $211,457,356$ & $63.86 \%$ \\
$(95,100]$ & 628 & $114,164,341$ & $325,621,697$ & $98.34 \%$ \\
NA & 48 & $5,504,979$ & $331,126,676$ & $100 \%$ \\
\hline
\end{tabular}

Notes: This table displays the number of county-sets and the population living in those countysets with different proportions of excess deaths assigned to COVID-19. For example, 93 counties had between 70 and $75 \%$ of excess deaths assigned to COVID-19, representing 37.3 million U.S. residents. Cumulatively, 91.2 million U.S. residents live in areas with less than $75 \%$ assignment of excess deaths to COVID-19. 


\section{Appendix B: Details on Model Selection and Estimation}

To select our primary specification for a distribution and link function, we computed both in-sample and out-of-sample predictive accuracy for a number of candidate specifications. Specifically, we estimated for three canonical GLM specifications for continuous and count data: Gaussian-identity, Poisson, and gamma models using data from 2010-2018. ${ }^{9}$ We then used the estimated model parameters to make out-of-sample predictions of 2019 mortality, and computed the mean-squared prediction error and mean absolute prediction error for each. Some prior studies have used a simple average of mortality in prior years to compute excess mortality in 2020. This has the advantage of being much simpler than fitting a high-dimensional GLM, although it will fail to capture trends, and may overweight more recent years. To gauge the improvement (if any) of using a regression model rather than a prior mean to predict deaths, we compute the predictive accuracy using both a one-year lagged value as well as a five-year prior average. Table B1 reports the mean squared and mean absolute prediction error, both in-sample and out-of-sample, associated with each model. Unsurprisingly, all of the GLMs provide a much better fit in terms of in-sample deviations than lagged value or prior mean. The GLMs also perform better in predicting mortality rates in 2019. Overall, the Gaussian and Poisson GLMs perform similarly on both metrics, with the Poisson providing a slighly better fit both in and out of sample. Given these results, and the results of using alternative windows for estimation and prediction, we chose the Poisson specification as our primary specification. ${ }^{1011}$ To obtain predicted mortality in each county set in 2020, we apply our estimated parametric conditional expectation function to 2020 data.

\subsection{Uncertainty Intervals}

We also construct a $95 \%$ prediction interval around the predicted death rates for 2020 to help identify counties in which 2020 mortality falls outside the normal range of year-to-year fluctuations. Importantly, while the point estimates of predicted mortality do not depend on the particular variance function assumed, the prediction intervals depend on both the variance of estimated parameters and the variance of outcome variable. ${ }^{12}$ To compute the prediction intervals, we first compute cluster-robust standard errors (Wooldridge, 1999)[35] for the individual parameter estimates, and then perform a parametric bootstrap procedure over the parameter and outcome distributions. ${ }^{13}$

\footnotetext{
${ }^{9}$ The negative binomial model was infeasible to estimate with our high-dimensional fixed effects

${ }^{10}$ Using alternative years to predict mortality results in the same general pattern of results, but with the Poisson and Gaussian specifications flip-flopping in terms of out-of-sample accuracy.

${ }^{11}$ It is worth pointing out that, in addition to providing good fit and predictive performance, the Poisson model has attractive robustness properties. Most importantly, achieving consistent parameter estimates does not depend on the assumption of a Poisson distribution. Moreover, our model allows for overdispersion or underdispersion of the outcome variable and there is no restriction on time dependence within clusters. See, for example, Gourieroux, Monfort, and Trognon (1984)[34] and Wooldridge (1999)[35] for details.

${ }^{12}$ In the standard Poisson GLM, the variance of the outcome variable is assumed to be equal to the mean. In practice this is highly restrictive, and so variance functions that permit overdispersion are common. Cameron and Trivedi (2013)[36] detail a number such specifications.

${ }^{13}$ This procedure is based on Gelman and Hill (2006)[37]. Woolf et al. (2020) use a similar procedure in an analogous setting at the state level to generate predictive intervals. [38]
} 
Table B1: Prediction Accuracy of Different Models

\begin{tabular}{lrrrrrr}
\hline & \multicolumn{2}{c}{ In Sample } & & \multicolumn{2}{c}{ Out-of-Sample } \\
\cline { 2 - 3 } \cline { 5 - 6 } & Mean Sq. Err. & Mean Abs. Err. & & Mean Sq. Err. & Mean Abs. Err. \\
\hline Prior Year & 0.282 & 0.400 & & 0.274 & 0.391 \\
Prior 5-Year Mean & 0.329 & 0.444 & & 0.340 & 0.455 \\
Gaussian-Identity GLM & 0.108 & 0.246 & & 0.216 & 0.348 \\
Poisson GLM & 0.104 & 0.241 & & 0.215 & 0.347 \\
Gamma GLM & 0.106 & 0.244 & & 0.229 & 0.359 \\
\hline
\end{tabular}

Notes: Mean squared error and mean absolute error of different statistical models of mortality. Models are estimated on annual mortality data from the CDC Wonder database once from 2009 to 2017 and once from 2010 to 2018. Predictions and out-of-sample errors are then generated for 2018 and 2019 and averaged.

Table B2: Prediction Accuracy of Different Models

\begin{tabular}{rr}
\hline Window & Mean Sq. Err \\
\hline 9 & 0.215 \\
8 & 0.221 \\
7 & 0.229 \\
6 & 0.244 \\
5 & 0.262 \\
4 & 0.278 \\
\hline
\end{tabular}

Notes: Mean squared error of poisson GLM applied to different lookback windows. 
medRxiv preprint doi: https://doi.org/10.1101/2021.04.23.21255564; this version posted August 29, 2021. The copyright holder for this preprint

(which was not certified by peer review) is the author/funder, who has granted medRxiv a license to display the preprint in perpetuity.

\begin{abstract}
It is made available under a CC-BY-NC-ND 4.0 International license .
\end{abstract}

\title{
8 Appendix C: All Estimates
}

Table C1 below includes all of our primary estimates for each county-set. Mortality rates are in units per 100,000 person-years. All-cause and COVID-19 deaths are based on provisional data from the National Center for Health Statistics. Lower CI and upper CI denote the approximate lower and upper bounds on the $95 \%$ prediction interval around expected and excess deaths in 2020 . Note that the ratio of COVID-19 to excess deaths is negative in some cases, indicating that the point estimate on excess deaths is negative for the county-set. In these cases the ratio does not have a clear interpretation. 
Table C1: Primary Estimates for Each County-Set

\begin{tabular}{|c|c|c|c|c|c|c|c|c|c|}
\hline County-Set & $\begin{array}{l}\text { Expected } \\
\text { Deaths }\end{array}$ & $\begin{array}{l}\text { Expected } \\
\text { Deaths } 95 \% \text { CI }\end{array}$ & $\begin{array}{l}\text { Observed } \\
\text { Deaths }\end{array}$ & $\begin{array}{l}\text { Observed/ } \\
\text { Expected }\end{array}$ & $\begin{array}{l}\text { Excess } \\
\text { Deaths }\end{array}$ & $\begin{array}{l}\text { Excess Deaths } \\
95 \% \text { CI }\end{array}$ & $\begin{array}{l}\text { Excess Deaths } \\
\text { Per } 100,000\end{array}$ & $\begin{array}{l}\text { COVID-19 } \\
\text { Deaths }\end{array}$ & $\begin{array}{c}\text { COVID-19 to } \\
\text { Excess Ratio (\%) }\end{array}$ \\
\hline Autauga, AL & 558 & $(497,612)$ & 651 & 1.17 & 93 & $(39,154)$ & 167 & 59 & 63.4 \\
\hline Baldwin, AL & 2417 & $(2299,2540)$ & 2691 & 1.11 & 274 & $(151,392)$ & 120 & 179 & 65.3 \\
\hline $\begin{array}{l}\text { Barbour-Bullock-Henry, } \\
\text { AL }\end{array}$ & 667 & $(604,736)$ & 794 & 1.19 & 127 & $(58,190)$ & 245 & 88 & 69.3 \\
\hline $\begin{array}{l}\text { Bibb-Chilton-Coosa-Perry, } \\
\text { AL }\end{array}$ & 1044 & $(966,1122)$ & 1271 & 1.22 & 227 & $(149,305)$ & 265 & 174 & 76.7 \\
\hline Blount, AL & 751 & $(686,817)$ & 849 & 1.13 & 98 & $(32,163)$ & 170 & 97 & 99.0 \\
\hline Butler-Crenshaw-Pike, AL & 777 & $(709,845)$ & 987 & 1.27 & 210 & $(142,278)$ & 317 & 131 & 62.4 \\
\hline Calhoun, AL & 1572 & $(1481,1676)$ & 1783 & 1.13 & 211 & $(107,302)$ & 186 & 190 & 90.0 \\
\hline $\begin{array}{l}\text { Chambers-Cleburne- } \\
\text { Randolph, AL }\end{array}$ & 956 & $(885,1034)$ & 1190 & 1.24 & 234 & $(156,305)$ & 328 & 165 & 70.5 \\
\hline Cherokee-Etowah, AL & 1819 & $(1700,1925)$ & 2147 & 1.18 & 328 & $(222,447)$ & 256 & 267 & 81.4 \\
\hline $\begin{array}{l}\text { Choctaw-Clarke- } \\
\text { Washington, AL }\end{array}$ & 715 & $(657,780)$ & 833 & 1.17 & 118 & $(53,176)$ & 225 & 103 & 87.3 \\
\hline Clay-Talladega, AL & 1214 & $(1129,1293)$ & 1445 & 1.19 & 231 & $(152,316)$ & 250 & 179 & 77.5 \\
\hline Coffee-Geneva, AL & 959 & $(885,1031)$ & 1174 & 1.22 & 215 & $(143,289)$ & 275 & 134 & 62.3 \\
\hline Colbert, AL & 774 & $(706,841)$ & 817 & 1.06 & 43 & $(-24,111)$ & 78 & 88 & 204.7 \\
\hline $\begin{array}{l}\text { Conecuh-Marengo- } \\
\text { Monroe-Wilcox, AL }\end{array}$ & 904 & $(834,982)$ & 1050 & 1.16 & 146 & $(68,216)$ & 237 & 116 & 79.5 \\
\hline Convington-Escambia, AL & 1080 & $(999,1163)$ & 1281 & 1.19 & 201 & $(118,282)$ & 276 & 159 & 79.1 \\
\hline Cullman, AL & 1118 & $(1035,1200)$ & 1259 & 1.13 & 141 & $(59,224)$ & 167 & 129 & 91.5 \\
\hline Dale, AL & 572 & $(515,631)$ & 635 & 1.11 & 63 & $(4,120)$ & 130 & 92 & 146.0 \\
\hline Dallas-Lowndes, AL & 674 & $(612,744)$ & 810 & 1.20 & 136 & $(66,198)$ & 293 & 145 & 106.6 \\
\hline DeKalb, AL & 865 & $(795,939)$ & 1024 & 1.18 & 159 & $(85,229)$ & 223 & 130 & 81.8 \\
\hline Elmore, AL & 876 & $(804,949)$ & 1014 & 1.16 & 138 & $(65,210)$ & 167 & 102 & 73.9 \\
\hline Fayette-Lamar-Marion, AL & 842 & $(769,915)$ & 1051 & 1.25 & 209 & $(136,282)$ & 350 & 162 & 77.5 \\
\hline Franklin-Lawrence, AL & 774 & $(708,844)$ & 987 & 1.28 & 213 & $(143,279)$ & 335 & 120 & 56.3 \\
\hline $\begin{array}{l}\text { Greene-Hale-Pickens- } \\
\text { Sumter, AL }\end{array}$ & 690 & $(627,753)$ & 933 & 1.35 & 243 & $(180,306)$ & 444 & 131 & 53.9 \\
\hline Houston, AL & 1207 & $(1124,1296)$ & 1427 & 1.18 & 220 & $(131,303)$ & 208 & 179 & 81.4 \\
\hline Jackson, AL & 742 & $(681,806)$ & 897 & 1.21 & 155 & $(91,216)$ & 302 & 71 & 45.8 \\
\hline Jefferson, AL & 7480 & $(7224,7723)$ & 8509 & 1.14 & 1029 & $(786,1285)$ & 156 & 798 & 77.6 \\
\hline Lauderdale, AL & 1149 & $(1069,1230)$ & 1335 & 1.16 & 186 & $(105,266)$ & 202 & 136 & 73.1 \\
\hline Lee, AL & 1220 & $(1133,1306)$ & 1307 & 1.07 & 87 & $(1,174)$ & 52 & 153 & 175.9 \\
\hline Limestone, AL & 909 & $(839,981)$ & 1085 & 1.19 & 176 & $(104,246)$ & 175 & 105 & 59.7 \\
\hline Macon-Tallapoosa, AL & 833 & $(760,906)$ & 947 & 1.14 & 114 & $(41,187)$ & 196 & 125 & 109.6 \\
\hline Madison, $\mathrm{AL}$ & 3589 & $(3435,3726)$ & 3763 & 1.05 & 174 & $(37,328)$ & 46 & 232 & 133.3 \\
\hline Marshall, AL & 1207 & $(1117,1293)$ & 1414 & 1.17 & 207 & $(121,297)$ & 213 & 157 & 75.8 \\
\hline Mobile, AL & 4516 & $(4322,4694)$ & 5214 & 1.15 & 698 & $(520,892)$ & 170 & 503 & 72.1 \\
\hline Montgomery, AL & 2176 & $(2066,2293)$ & 2771 & 1.27 & 595 & $(478,705)$ & 267 & 356 & 59.8 \\
\hline Morgan, AL & 1461 & $(1358,1556)$ & 1735 & 1.19 & 274 & $(179,377)$ & 229 & 180 & 65.7 \\
\hline Russell, AL & 675 & $(613,735)$ & 750 & 1.11 & 75 & $(15,137)$ & 127 & 77 & 102.7 \\
\hline St. Clair, AL & 1027 & $(951,1102)$ & 1221 & 1.19 & 194 & $(119,270)$ & 215 & 142 & 73.2 \\
\hline Shelby, AL & 1623 & $(1526,1722)$ & 1841 & 1.13 & 218 & $(119,315)$ & 99 & 159 & 72.9 \\
\hline Tuscaloosa, AL & 1822 & $(1717,1929)$ & 2205 & 1.21 & 383 & $(276,488)$ & 181 & 265 & 69.2 \\
\hline Walker-Winston, AL & 1387 & $(1288,1483)$ & 1679 & 1.21 & 292 & $(196,391)$ & 337 & 213 & 72.9 \\
\hline Anchorage, AK & 1835 & $(1727,1929)$ & 2076 & 1.13 & 241 & $(147,349)$ & 84 & 139 & 57.7 \\
\hline Fairbanks North Star, AK & 495 & $(443,545)$ & 544 & 1.10 & 49 & $(-1,101)$ & 50 & 21 & 42.9 \\
\hline Kenai Peninsula, AK & 504 & $(453,560)$ & 470 & 0.93 & -34 & $(-90,17)$ & -59 & 21 & NA \\
\hline Matanuska-Susitna, AK & 656 & $(600,723)$ & 761 & 1.16 & 105 & $(38,161)$ & 96 & 34 & 32.4 \\
\hline Northern Alaska, AK & 376 & $(331,422)$ & 380 & 1.01 & 4 & $(-42,49)$ & 9 & 25 & 625.0 \\
\hline Southeast Alaska, AK & 544 & $(488,599)$ & 552 & 1.01 & 8 & $(-47,64)$ & 11 & 25 & 312.5 \\
\hline Southwest Alaska, AK & 276 & $(238,314)$ & 317 & 1.15 & 41 & $(3,79)$ & 84 & 25 & 61.0 \\
\hline Apache, AZ & 784 & $(716,849)$ & 1172 & 1.49 & 388 & $(323,456)$ & 537 & 315 & 81.2 \\
\hline Cochise-Graham-Greenee, & 1875 & $(1773,1976)$ & 2092 & 1.12 & 217 & $(116,319)$ & 122 & 240 & 110.6 \\
\hline
\end{tabular}


Table C1: Primary Estimates for Each County-Set

\begin{tabular}{|c|c|c|c|c|c|c|c|c|c|}
\hline County-Set & $\begin{array}{l}\text { Expected } \\
\text { Deaths }\end{array}$ & $\begin{array}{l}\text { Expected } \\
\text { Deaths } 95 \% \text { CI }\end{array}$ & $\begin{array}{l}\text { Observed } \\
\text { Deaths }\end{array}$ & $\begin{array}{l}\text { Observed/ } \\
\text { Expected }\end{array}$ & $\begin{array}{l}\text { Excess } \\
\text { Deaths }\end{array}$ & $\begin{array}{l}\text { Excess Deaths } \\
95 \% \mathrm{CI}\end{array}$ & $\begin{array}{l}\text { Excess Deaths } \\
\text { Per } 100,000\end{array}$ & $\begin{array}{l}\text { COVID-19 } \\
\text { Deaths }\end{array}$ & $\begin{array}{l}\text { COVID-19 to } \\
\text { Excess Ratio (\%) }\end{array}$ \\
\hline Coconino, $\mathrm{AZ}$ & 926 & $(856,996)$ & 1178 & 1.27 & 252 & $(182,322)$ & 174 & 219 & 86.9 \\
\hline Gila, AZ & 841 & $(777,914)$ & 975 & 1.16 & 134 & $(61,198)$ & 246 & 131 & 97.8 \\
\hline La Paz-Yavapai, AZ & 3704 & $(3542,3889)$ & 4084 & 1.10 & 380 & $(195,542)$ & 145 & 316 & 83.2 \\
\hline Maricopa, AZ & 34322 & $(33668,35028)$ & 40958 & 1.19 & 6636 & $(5930,7290)$ & 145 & 4829 & 72.8 \\
\hline Mohave, AZ & 3652 & $(3461,3829)$ & 4041 & 1.11 & 389 & $(212,580)$ & 182 & 363 & 93.3 \\
\hline Navajo, AZ & 1217 & $(1134,1302)$ & 1737 & 1.43 & 520 & $(435,603)$ & 460 & 390 & 75.0 \\
\hline Pima-Santa Cruz, AZ & 10686 & $(10377,11016)$ & 12760 & 1.19 & 2074 & $(1744,2383)$ & 187 & 1380 & 66.5 \\
\hline Pinal, AZ & 3620 & $(3483,3770)$ & 4291 & 1.19 & 671 & $(521,808)$ & 140 & 431 & 64.2 \\
\hline Yuma, AZ & 1678 & $(1583,1773)$ & 2453 & 1.46 & 775 & $(680,870)$ & 357 & 568 & 73.3 \\
\hline Arkansas & 733 & $(672,799)$ & 910 & 1.24 & 177 & $(111,238)$ & 363 & 90 & 50.8 \\
\hline \multicolumn{10}{|c|}{$\begin{array}{l}\text { Monroe-Phillips-Prairie, } \\
\text { AR }\end{array}$} \\
\hline Baxter-Marion, AR & 1001 & $(919,1088)$ & 1166 & 1.16 & 165 & $(78,247)$ & 277 & 105 & 63.6 \\
\hline Benton, AR & 2004 & $(1893,2106)$ & 2301 & 1.15 & 297 & $(195,408)$ & 104 & 294 & 99.0 \\
\hline $\begin{array}{l}\text { Boone-Carroll-Madison, } \\
\text { AR }\end{array}$ & 1044 & $(973,1127)$ & 1124 & 1.08 & 80 & $(-3,151)$ & 96 & 118 & 147.5 \\
\hline $\begin{array}{l}\text { Bradley-Calhoun- } \\
\text { Cleveland-Union, AR }\end{array}$ & 899 & $(827,974)$ & 1023 & 1.14 & 124 & $(49,196)$ & 198 & 134 & 108.1 \\
\hline $\begin{array}{l}\text { Clark-Dallas-Ouachita, } \\
\text { AR }\end{array}$ & 685 & $(625,754)$ & 825 & 1.20 & 140 & $(71,200)$ & 269 & 76 & 54.3 \\
\hline Clay-Greene, AR & 758 & $(695,828)$ & 833 & 1.10 & 75 & $(5,138)$ & 123 & 92 & 122.7 \\
\hline $\begin{array}{l}\text { Columbia-Lafayette- } \\
\text { Miller, AR }\end{array}$ & 899 & $(826,975)$ & 1092 & 1.21 & 193 & $(117,266)$ & 266 & 121 & 62.7 \\
\hline Conway-Pope, AR & 942 & $(867,1012)$ & 1056 & 1.12 & 114 & $(44,189)$ & 133 & 78 & 68.4 \\
\hline Craighead, AR & 993 & $(922,1072)$ & 1183 & 1.19 & 190 & $(111,261)$ & 170 & 135 & 71.1 \\
\hline Crawford, AR & 676 & $(610,739)$ & 785 & 1.16 & 109 & $(46,175)$ & 169 & 65 & 59.6 \\
\hline Crittenden, AR & 526 & $(473,580)$ & 638 & 1.21 & 112 & $(58,165)$ & 236 & 90 & 80.4 \\
\hline Cross-Lee-St. Francis, AR & 616 & $(559,675)$ & 751 & 1.22 & 135 & $(76,192)$ & 272 & 88 & 65.2 \\
\hline Faulkner, AR & 1104 & $(1026,1182)$ & 1122 & 1.02 & 18 & $(-60,96)$ & 14 & 92 & 511.1 \\
\hline $\begin{array}{l}\text { Franklin-Johnson-Logan, } \\
\text { AR }\end{array}$ & 829 & $(761,899)$ & 930 & 1.12 & 101 & $(31,169)$ & 151 & 65 & 64.4 \\
\hline Garland, AR & 1471 & $(1371,1562)$ & 1604 & 1.09 & 133 & $(42,233)$ & 133 & 152 & 114.3 \\
\hline Grant-Hot Spring, AR & 671 & $(608,731)$ & 746 & 1.11 & 75 & $(15,138)$ & 144 & 77 & 102.7 \\
\hline $\begin{array}{l}\text { Hempstead-Howard- } \\
\text { Nevada-Pike, AR }\end{array}$ & 695 & $(633,762)$ & 746 & 1.07 & 51 & $(-16,113)$ & 94 & 84 & 164.7 \\
\hline $\begin{array}{l}\text { Independence-Jackson- } \\
\text { Woodruff, AR }\end{array}$ & 765 & $(698,828)$ & 939 & 1.23 & 174 & $(111,241)$ & 287 & 117 & 67.2 \\
\hline Jefferson, AR & 840 & $(768,909)$ & 1032 & 1.23 & 192 & $(123,264)$ & 292 & 139 & 72.4 \\
\hline $\begin{array}{l}\text { Little River-Polk-Sevier, } \\
\text { AR }\end{array}$ & 623 & $(566,685)$ & 745 & 1.20 & 122 & $(60,179)$ & 248 & 129 & 105.7 \\
\hline Lonoke, AR & 737 & $(670,807)$ & 804 & 1.09 & 67 & $(-3,134)$ & 89 & 88 & 131.3 \\
\hline Mississippi-Poinsett, AR & 878 & $(804,954)$ & 1089 & 1.24 & 211 & $(135,285)$ & 334 & 149 & 70.6 \\
\hline $\begin{array}{l}\text { Montgomery-Perry-Scott- } \\
\text { Yell, AR }\end{array}$ & 704 & $(640,767)$ & 761 & 1.08 & 57 & $(-6,121)$ & 111 & 94 & 164.9 \\
\hline Pulaski, AR & 3901 & $(3728,4069)$ & 4479 & 1.15 & 578 & $(410,751)$ & 148 & 394 & 68.2 \\
\hline Saline, AR & 1299 & $(1213,1388)$ & 1316 & 1.01 & 17 & $(-72,103)$ & 14 & 119 & 700.0 \\
\hline Sebastian, AR & 1356 & $(1257,1445)$ & 1569 & 1.16 & 213 & $(124,312)$ & 167 & 136 & 63.8 \\
\hline $\begin{array}{l}\text { Sharp and Nearby Coun- } \\
\text { ties, AR }\end{array}$ & 1246 & $(1163,1334)$ & 1313 & 1.05 & 67 & $(-21,150)$ & 85 & 161 & 240.3 \\
\hline Southeast Arkansas, AR & 987 & $(912,1064)$ & 1122 & 1.14 & 135 & $(58,210)$ & 187 & 120 & 88.9 \\
\hline $\begin{array}{l}\text { Van Buren and Nearby } \\
\text { Counties, AR }\end{array}$ & 1054 & $(968,1135)$ & 1157 & 1.10 & 103 & $(22,189)$ & 148 & 117 & 113.6 \\
\hline Washington, AR & 1792 & $(1692,1900)$ & 1943 & 1.08 & 151 & $(43,251)$ & 62 & 233 & 154.3 \\
\hline White, AR & 881 & $(809,951)$ & 995 & 1.13 & 114 & $(44,186)$ & 145 & 62 & 54.4 \\
\hline Alameda, CA & 10130 & $(9827,10395)$ & 11237 & 1.11 & 1107 & $(842,1410)$ & 66 & 689 & 62.2 \\
\hline
\end{tabular}


Table C1: Primary Estimates for Each County-Set

\begin{tabular}{|c|c|c|c|c|c|c|c|c|c|}
\hline County-Set & $\begin{array}{l}\text { Expected } \\
\text { Deaths }\end{array}$ & $\begin{array}{l}\text { Expected } \\
\text { Deaths } 95 \% \text { CI }\end{array}$ & $\begin{array}{l}\text { Observed } \\
\text { Deaths }\end{array}$ & $\begin{array}{l}\text { Observed/ } \\
\text { Expected }\end{array}$ & $\begin{array}{l}\text { Excess } \\
\text { Deaths }\end{array}$ & $\begin{array}{l}\text { Excess Deaths } \\
95 \% \mathrm{CI}\end{array}$ & $\begin{array}{l}\text { Excess Deaths } \\
\text { Per } 100,000\end{array}$ & $\begin{array}{l}\text { COVID-19 } \\
\text { Deaths }\end{array}$ & $\begin{array}{l}\text { COVID-19 to } \\
\text { Excess Ratio (\%) }\end{array}$ \\
\hline $\begin{array}{l}\text { Alpine and Nearby Coun- } \\
\text { ties, CA }\end{array}$ & 1312 & $(1221,1406)$ & 1383 & 1.05 & 71 & $(-23,162)$ & 60 & 92 & 129.6 \\
\hline Butte, CA & 2562 & $(2439,2692)$ & 2343 & 0.91 & -219 & $(-349,-96)$ & -93 & 148 & NA \\
\hline Colusa-Lake, CA & 1074 & $(998,1156)$ & 1035 & 0.96 & -39 & $(-121,37)$ & -45 & 62 & NA \\
\hline Contra Costa, CA & 8079 & $(7835,8324)$ & 8800 & 1.09 & 721 & $(476,965)$ & 62 & 480 & 66.6 \\
\hline Del Norte-Humboldt- & 1882 & $(1775,1989)$ & 1897 & 1.01 & 15 & $(-92,122)$ & 9 & 35 & 233.3 \\
\hline Trinity, CA & & & & & & & & & \\
\hline El Dorado, CA & 1693 & $(1594,1794)$ & 1809 & 1.07 & 116 & $(15,215)$ & 60 & 78 & 67.2 \\
\hline Fresno, CA & 7325 & $(7097,7549)$ & 8763 & 1.20 & 1438 & $(1214,1666)$ & 142 & 1017 & 70.7 \\
\hline Glenn-Tehama, CA & 1072 & $(998,1154)$ & 1069 & 1.00 & -3 & $(-85,71)$ & -3 & 72 & NA \\
\hline Imperial, CA & 1187 & $(1108,1271)$ & 1892 & 1.59 & 705 & $(621,784)$ & 385 & 537 & 76.2 \\
\hline Kern, CA & 6531 & $(6312,6745)$ & 7667 & 1.17 & 1136 & $(922,1355)$ & 125 & 761 & 67.0 \\
\hline Kings, CA & 926 & $(853,1003)$ & 1071 & 1.16 & 145 & $(68,218)$ & 95 & 129 & 89.0 \\
\hline Lassen-Plumas-Sierra, CA & 537 & $(483,592)$ & 596 & 1.11 & 59 & $(4,113)$ & 113 & 22 & 37.3 \\
\hline Los Angeles, CA & 65748 & $(64670,66811)$ & 81703 & 1.24 & 15955 & $(14892,17033)$ & 158 & 11573 & 72.5 \\
\hline Madera, CA & 1168 & $(1088,1249)$ & 1326 & 1.14 & 158 & $(77,238)$ & 98 & 184 & 116.5 \\
\hline Marin, CA & 1965 & $(1856,2076)$ & 2119 & 1.08 & 154 & $(43,263)$ & 59 & 132 & 85.7 \\
\hline Mariposa-Tuolumne, CA & 961 & $(885,1046)$ & 965 & 1.00 & 4 & $(-81,80)$ & 5 & 46 & 1150.0 \\
\hline Mendocino, CA & 878 & $(809,948)$ & 1041 & 1.19 & 163 & $(93,232)$ & 187 & 38 & 23.3 \\
\hline Merced, CA & 1905 & $(1792,2006)$ & 2278 & 1.20 & 373 & $(272,486)$ & 133 & 282 & 75.6 \\
\hline Modoc-Siskiyou, CA & 726 & $(657,794)$ & 753 & 1.04 & 27 & $(-41,96)$ & 52 & 22 & 81.5 \\
\hline Monterey, CA & 2723 & $(2601,2838)$ & 3026 & 1.11 & 303 & $(188,425)$ & 69 & 265 & 87.5 \\
\hline Napa, CA & 1233 & $(1145,1313)$ & 1254 & 1.02 & 21 & $(-59,109)$ & 16 & 47 & 223.8 \\
\hline Nevada, CA & 1113 & $(1033,1196)$ & 1174 & 1.05 & 61 & $(-22,141)$ & 61 & 70 & 114.8 \\
\hline Orange, CA & 20966 & $(20503,21449)$ & 23626 & 1.13 & 2660 & $(2177,3123)$ & 83 & 2647 & 99.5 \\
\hline Placer, CA & 3515 & $(3369,3668)$ & 3583 & 1.02 & 68 & $(-85,214)$ & 17 & 187 & 275.0 \\
\hline Riverside, CA & 18133 & $(17659,18562)$ & 21744 & 1.20 & 3611 & $(3182,4085)$ & 143 & 2849 & 78.9 \\
\hline Sacramento, CA & 12329 & $(11989,12673)$ & 13454 & 1.09 & 1125 & $(781,1465)$ & 72 & 1052 & 93.5 \\
\hline San Benito, CA & 394 & $(346,444)$ & 423 & 1.07 & 29 & $(-21,77)$ & 45 & 35 & 120.7 \\
\hline San Bernardino, CA & 15505 & $(15171,15859)$ & 19383 & 1.25 & 3878 & $(3524,4212)$ & 176 & 2884 & 74.4 \\
\hline San Diego, CA & 22308 & $(21772,22841)$ & 24788 & 1.11 & 2480 & $(1947,3016)$ & 74 & 1883 & 75.9 \\
\hline San Francisco, CA & 5804 & $(5615,6006)$ & 6351 & 1.09 & 547 & $(345,736)$ & 61 & 263 & 48.1 \\
\hline San Joaquin, CA & 5959 & $(5755,6151)$ & 7038 & 1.18 & 1079 & $(887,1283)$ & 140 & 855 & 79.2 \\
\hline San Luis Obispo, CA & 2516 & $(2393,2649)$ & 2512 & 1.00 & -4 & $(-137,119)$ & -2 & 122 & NA \\
\hline San Mateo, CA & 4722 & $(4537,4906)$ & 5028 & 1.06 & 306 & $(122,491)$ & 40 & 268 & 87.6 \\
\hline Santa Barbara, CA & 3308 & $(3169,3458)$ & 3499 & 1.06 & 191 & $(41,330)$ & 43 & 188 & 98.4 \\
\hline Santa Clara, CA & 10448 & $(10160,10715)$ & 11468 & 1.10 & 1020 & $(753,1308)$ & 52 & 886 & 86.9 \\
\hline Santa Cruz, CA & 1746 & $(1645,1848)$ & 1889 & 1.08 & 143 & $(41,244)$ & 52 & 129 & 90.2 \\
\hline Shasta, CA & 2365 & $(2251,2487)$ & 2451 & 1.04 & 86 & $(-36,200)$ & 47 & 112 & 130.2 \\
\hline Solano, CA & 3674 & $(3524,3828)$ & 3764 & 1.02 & 90 & $(-64,240)$ & 20 & 198 & 220.0 \\
\hline Sonoma, CA & 4168 & $(4008,4341)$ & 4440 & 1.07 & 272 & $(99,432)$ & 55 & 221 & 81.2 \\
\hline Stanislaus, CA & 4615 & $(4443,4796)$ & 5353 & 1.16 & 738 & $(557,910)$ & 132 & 685 & 92.8 \\
\hline Sutter, CA & 896 & $(828,969)$ & 944 & 1.05 & 48 & $(-25,116)$ & 49 & 66 & 137.5 \\
\hline Tulare, CA & 3243 & $(3107,3381)$ & 3928 & 1.21 & 685 & $(547,821)$ & 145 & 533 & 77.8 \\
\hline Ventura, CA & 6053 & $(5852,6272)$ & 6615 & 1.09 & 562 & $(343,763)$ & 66 & 437 & 77.8 \\
\hline Yolo, CA & 1381 & $(1296,1468)$ & 1506 & 1.09 & 125 & $(38,210)$ & 56 & 127 & 101.6 \\
\hline Yuba, CA & 730 & $(668,795)$ & 677 & 0.93 & -53 & $(-118,9)$ & -65 & 32 & NA \\
\hline Adams, CO & 3130 & $(2997,3265)$ & 3702 & 1.18 & 572 & $(437,705)$ & 108 & 502 & 87.8 \\
\hline Arapahoe, CO & 4113 & $(3953,4254)$ & 4923 & 1.20 & 810 & $(669,970)$ & 122 & 568 & 70.1 \\
\hline Boulder, CO & 2012 & $(1902,2127)$ & 2166 & 1.08 & 154 & $(39,264)$ & 47 & 167 & 108.4 \\
\hline Broomfield, CO & 410 & $(364,456)$ & 530 & 1.29 & 120 & $(74,166)$ & 168 & 61 & 50.8 \\
\hline Central Colorado, $\mathrm{CO}$ & 435 & $(387,486)$ & 468 & 1.08 & 33 & $(-18,81)$ & 46 & 33 & 100.0 \\
\hline Custer-Fremont, CO & 657 & $(597,719)$ & 679 & 1.03 & 22 & $(-40,82)$ & 42 & 37 & 168.2 \\
\hline Delta-Montrose, CO & 913 & $(839,983)$ & 944 & 1.03 & 31 & $(-39,105)$ & 42 & 63 & 203.2 \\
\hline Denver, CO & 4632 & $(4461,4817)$ & 5758 & 1.24 & 1126 & $(941,1297)$ & 152 & 734 & 65.2 \\
\hline Douglas, CO & 1634 & $(1542,1726)$ & 1922 & 1.18 & 288 & $(196,380)$ & 81 & 193 & 67.0 \\
\hline
\end{tabular}


Table C1: Primary Estimates for Each County-Set

\begin{tabular}{|c|c|c|c|c|c|c|c|c|c|}
\hline County-Set & $\begin{array}{l}\text { Expected } \\
\text { Deaths }\end{array}$ & $\begin{array}{l}\text { Expected } \\
\text { Deaths } 95 \% \text { CI }\end{array}$ & $\begin{array}{l}\text { Observed } \\
\text { Deaths }\end{array}$ & $\begin{array}{l}\text { Observed/ } \\
\text { Expected }\end{array}$ & $\begin{array}{l}\text { Excess } \\
\text { Deaths }\end{array}$ & $\begin{array}{l}\text { Excess Deaths } \\
95 \% \mathrm{CI}\end{array}$ & $\begin{array}{l}\text { Excess Deaths } \\
\text { Per } 100,000\end{array}$ & $\begin{array}{l}\text { COVID-19 } \\
\text { Deaths }\end{array}$ & $\begin{array}{c}\text { COVID-19 to } \\
\text { Excess Ratio (\%) }\end{array}$ \\
\hline Eagle, CO & 159 & $(130,187)$ & 159 & 1.00 & 0 & $(-28,29)$ & 1 & 14 & NA \\
\hline East Central Colorado, CO & 443 & $(395,491)$ & 523 & 1.18 & 80 & $(32,128)$ & 145 & 51 & 63.7 \\
\hline El Paso, CO & 5431 & $(5238,5620)$ & 6176 & 1.14 & 745 & $(556,938)$ & 101 & 595 & 79.9 \\
\hline Garfield, CO & 395 & $(350,443)$ & 394 & 1.00 & -1 & $(-49,44)$ & -2 & 40 & NA \\
\hline Jefferson, CO & 4705 & $(4528,4884)$ & 5300 & 1.13 & 595 & $(416,772)$ & 101 & 579 & 97.3 \\
\hline La Plata-Montezuma, CO & 649 & $(588,709)$ & 728 & 1.12 & 79 & $(19,140)$ & 95 & 46 & 58.2 \\
\hline Larimer, CO & 2547 & $(2429,2667)$ & 2663 & 1.05 & 116 & $(-4,234)$ & 32 & 139 & 119.8 \\
\hline $\begin{array}{l}\text { Logan-Morgan-Phillips- } \\
\text { Sedgwick, CO }\end{array}$ & 590 & $(534,648)$ & 786 & 1.33 & 196 & $(138,252)$ & 344 & 146 & 74.5 \\
\hline Mesa, CO & 1663 & $(1565,1762)$ & 1801 & 1.08 & 138 & $(39,236)$ & 88 & 141 & 102.2 \\
\hline Northwest Colorado, CO & 396 & $(349,442)$ & 450 & 1.14 & 54 & $(8,101)$ & 85 & 57 & 105.6 \\
\hline $\begin{array}{l}\text { Park and Nearby Counties, } \\
\text { CO }\end{array}$ & 494 & $(443,545)$ & 524 & 1.06 & 30 & $(-21,81)$ & 33 & 31 & 103.3 \\
\hline Pueblo, CO & 1935 & $(1825,2049)$ & 2328 & 1.20 & 393 & $(279,503)$ & 231 & 302 & 76.8 \\
\hline Southeast Colorado, CO & 1050 & $(975,1134)$ & 1218 & 1.16 & 168 & $(84,243)$ & 199 & 129 & 76.8 \\
\hline Southwest Colorado, CO & 515 & $(460,573)$ & 535 & 1.04 & 20 & $(-38,75)$ & 33 & 43 & 215.0 \\
\hline Weld, CO & 2012 & $(1909,2121)$ & 2233 & 1.11 & 221 & $(112,324)$ & 66 & 295 & 133.5 \\
\hline Fairfield, CT & 6990 & $(6752,7230)$ & 8790 & 1.26 & 1800 & $(1560,2038)$ & 191 & 1726 & 95.9 \\
\hline Hartford, CT & 8324 & $(8087,8584)$ & 10038 & 1.21 & 1714 & $(1454,1951)$ & 193 & 1859 & 108.5 \\
\hline Litchfield, CT & 1933 & $(1820,2040)$ & 2209 & 1.14 & 276 & $(169,389)$ & 154 & 261 & 94.6 \\
\hline Middlesex, CT & 1668 & $(1565,1763)$ & 1806 & 1.08 & 138 & $(43,241)$ & 85 & 241 & 174.6 \\
\hline New Haven, CT & 8107 & $(7840,8368)$ & 9727 & 1.20 & 1620 & $(1359,1887)$ & 189 & 1606 & 99.1 \\
\hline New London, CT & 2621 & $(2486,2752)$ & 2787 & 1.06 & 166 & $(35,301)$ & 63 & 300 & 180.7 \\
\hline Tolland, CT & 1165 & $(1087,1241)$ & 1204 & 1.03 & 39 & $(-37,117)$ & 26 & 128 & 328.2 \\
\hline Windham, CT & 1179 & $(1092,1266)$ & 1270 & 1.08 & 91 & $(4,178)$ & 77 & 93 & 102.2 \\
\hline Kent, DE & 1802 & $(1702,1905)$ & 2026 & 1.12 & 224 & $(121,324)$ & 123 & 197 & 87.9 \\
\hline New Castle, DE & 5104 & $(4915,5307)$ & 5761 & 1.13 & 657 & $(454,846)$ & 116 & 511 & 77.8 \\
\hline Sussex, DE & 2962 & $(2819,3106)$ & 3284 & 1.11 & 322 & $(178,465)$ & 134 & 330 & 102.5 \\
\hline District of Columbia, DC & 5035 & $(4839,5223)$ & 6198 & 1.23 & 1163 & $(975,1359)$ & 162 & 885 & 76.1 \\
\hline Alachua, FL & 2115 & $(2003,2234)$ & 2309 & 1.09 & 194 & $(75,306)$ & 70 & 155 & 79.9 \\
\hline Baker-Bradford-Union, FL & 908 & $(834,982)$ & 962 & 1.06 & 54 & $(-20,128)$ & 75 & 124 & 229.6 \\
\hline Bay, FL & 2136 & $(2022,2253)$ & 2130 & 1.00 & -6 & $(-123,108)$ & -3 & 204 & NA \\
\hline Brevard, FL & 8204 & $(7924,8504)$ & 8383 & 1.02 & 179 & $(-121,459)$ & 29 & 518 & 289.4 \\
\hline Broward, FL & 15507 & $(15131,15883)$ & 17985 & 1.16 & 2478 & $(2102,2854)$ & 125 & 1940 & 78.3 \\
\hline Charlotte, FL & 2888 & $(2737,3039)$ & 3183 & 1.10 & 295 & $(144,446)$ & 154 & 268 & 90.8 \\
\hline Citrus, FL & 2873 & $(2711,3031)$ & 3219 & 1.12 & 346 & $(188,508)$ & 226 & 241 & 69.7 \\
\hline Clay, FL & 2013 & $(1904,2123)$ & 2207 & 1.10 & 194 & $(84,303)$ & 87 & 157 & 80.9 \\
\hline Collier, FL & 3675 & $(3515,3830)$ & 4044 & 1.10 & 369 & $(214,529)$ & 94 & 363 & 98.4 \\
\hline Columbia, FL & 860 & $(795,936)$ & 968 & 1.13 & 108 & $(32,173)$ & 150 & 103 & 95.4 \\
\hline DeSoto-Hardee, FL & 647 & $(588,705)$ & 700 & 1.08 & 53 & $(-5,112)$ & 81 & 80 & 150.9 \\
\hline Dixie-Gilchrist-Levy, FL & 1058 & $(978,1138)$ & 1119 & 1.06 & 61 & $(-19,141)$ & 78 & 64 & 104.9 \\
\hline Duval, FL & 9037 & $(8752,9310)$ & 10131 & 1.12 & 1094 & $(821,1379)$ & 112 & 762 & 69.7 \\
\hline Escambia, FL & 3696 & $(3527,3856)$ & 4225 & 1.14 & 529 & $(369,698)$ & 165 & 341 & 64.5 \\
\hline Flagler, FL & 1509 & $(1412,1605)$ & 1613 & 1.07 & 104 & $(8,201)$ & 90 & 58 & 55.8 \\
\hline Gadsden-Leon, FL & 2543 & $(2422,2664)$ & 3004 & 1.18 & 461 & $(340,582)$ & 135 & 229 & 49.7 \\
\hline $\begin{array}{l}\text { Glades-Hendry- } \\
\text { Okeechobee, FL }\end{array}$ & 1008 & $(934,1083)$ & 1082 & 1.07 & 74 & $(-1,148)$ & 75 & 129 & 174.3 \\
\hline $\begin{array}{l}\text { Hamilton-Lafayette- } \\
\text { Suwannee, FL }\end{array}$ & 873 & $(798,945)$ & 993 & 1.14 & 120 & $(48,195)$ & 176 & 115 & 95.8 \\
\hline Hernando, FL & 3004 & $(2850,3167)$ & 3175 & 1.06 & 171 & $(8,325)$ & 86 & 236 & 138.0 \\
\hline Highlands, FL & 1735 & $(1621,1845)$ & 1869 & 1.08 & 134 & $(24,248)$ & 124 & 195 & 145.5 \\
\hline Hillsborough, FL & 11309 & $(10999,11633)$ & 12845 & 1.14 & 1536 & $(1212,1846)$ & 103 & 1088 & 70.8 \\
\hline Holmes-Walton, FL & 1081 & $(1003,1155)$ & 1133 & 1.05 & 52 & $(-22,130)$ & 54 & 82 & 157.7 \\
\hline Indian River, FL & 2256 & $(2130,2387)$ & 2387 & 1.06 & 131 & $(0,257)$ & 80 & 152 & 116.0 \\
\hline Jackson-Washington, FL & 1083 & $(1006,1168)$ & 1133 & 1.05 & 50 & $(-35,127)$ & 67 & 143 & 286.0 \\
\hline
\end{tabular}


Table C1: Primary Estimates for Each County-Set

\begin{tabular}{|c|c|c|c|c|c|c|c|c|c|}
\hline County-Set & $\begin{array}{l}\text { Expected } \\
\text { Deaths }\end{array}$ & $\begin{array}{l}\text { Expected } \\
\text { Deaths } 95 \% \text { CI }\end{array}$ & $\begin{array}{l}\text { Observed } \\
\text { Deaths }\end{array}$ & $\begin{array}{l}\text { Observed/ } \\
\text { Expected }\end{array}$ & $\begin{array}{l}\text { Excess } \\
\text { Deaths }\end{array}$ & $\begin{array}{l}\text { Excess Deaths } \\
95 \% \text { CI }\end{array}$ & $\begin{array}{l}\text { Excess Deaths } \\
\text { Per } 100,000\end{array}$ & $\begin{array}{l}\text { COVID-19 } \\
\text { Deaths }\end{array}$ & $\begin{array}{l}\text { COVID-19 to } \\
\text { Excess Ratio (\%) }\end{array}$ \\
\hline $\begin{array}{l}\text { Jefferson-Madison-Taylor, } \\
\text { FL }\end{array}$ & 708 & $(641,776)$ & 874 & 1.23 & 166 & $(98,233)$ & 304 & 88 & 53.0 \\
\hline Lake, FL & 4904 & $(4699,5105)$ & 5147 & 1.05 & 243 & $(42,448)$ & 64 & 321 & 132.1 \\
\hline Lee, FL & 7806 & $(7536,8057)$ & 8620 & 1.10 & 814 & $(563,1084)$ & 104 & 680 & 83.5 \\
\hline $\begin{array}{l}\text { Liberty and Nearby Coun- } \\
\text { ties, FL }\end{array}$ & 961 & $(888,1037)$ & 1096 & 1.14 & 135 & $(59,208)$ & 157 & 96 & 71.1 \\
\hline Manatee, FL & 4446 & $(4269,4627)$ & 4834 & 1.09 & 388 & $(207,565)$ & 94 & 450 & 116.0 \\
\hline Marion, FL & 5632 & $(5394,5871)$ & 6145 & 1.09 & 513 & $(274,751)$ & 138 & 489 & 95.3 \\
\hline Martin, FL & 2051 & $(1930,2165)$ & 2338 & 1.14 & 287 & $(173,408)$ & 176 & 184 & 64.1 \\
\hline Miami-Dade, FL & 21249 & $(20744,21736)$ & 25621 & 1.21 & 4372 & $(3885,4877)$ & 157 & 4092 & 93.6 \\
\hline Monroe, FL & 717 & $(655,781)$ & 770 & 1.07 & 53 & $(-11,115)$ & 74 & 42 & 79.2 \\
\hline Nassau, FL & 1068 & $(987,1145)$ & 1079 & 1.01 & 11 & $(-66,92)$ & 12 & 71 & 645.5 \\
\hline Okaloosa, FL & 2022 & $(1909,2130)$ & 2231 & 1.10 & 209 & $(101,322)$ & 98 & 221 & 105.7 \\
\hline Orange, FL & 8857 & $(8612,9108)$ & 9855 & 1.11 & 998 & $(747,1243)$ & 70 & 818 & 82.0 \\
\hline Osceola, FL & 2707 & $(2572,2838)$ & 3004 & 1.11 & 297 & $(166,432)$ & 75 & 298 & 100.3 \\
\hline Palm Beach, FL & 15322 & $(14878,15748)$ & 17273 & 1.13 & 1951 & $(1525,2395)$ & 129 & 1806 & 92.6 \\
\hline Pasco, FL & 6791 & $(6536,7037)$ & 7259 & 1.07 & 468 & $(222,723)$ & 82 & 420 & 89.7 \\
\hline Pinellas, FL & 12411 & $(11984,12821)$ & 13290 & 1.07 & 879 & $(469,1306)$ & 89 & 935 & 106.4 \\
\hline Polk, FL & 7818 & $(7546,8073)$ & 8595 & 1.10 & 777 & $(522,1049)$ & 103 & 760 & 97.8 \\
\hline Putnam, FL & 1100 & $(1018,1187)$ & 1168 & 1.06 & 68 & $(-19,150)$ & 90 & 78 & 114.7 \\
\hline St. Johns, FL & 2312 & $(2186,2429)$ & 2362 & 1.02 & 50 & $(-67,176)$ & 18 & 111 & 222.0 \\
\hline St. Lucie, FL & 3635 & $(3476,3785)$ & 3982 & 1.10 & 347 & $(197,506)$ & 103 & 381 & 109.8 \\
\hline Santa Rosa, FL & 1785 & $(1690,1890)$ & 1866 & 1.05 & 81 & $(-24,176)$ & 42 & 134 & 165.4 \\
\hline Sarasota, FL & 6154 & $(5911,6390)$ & 6800 & 1.10 & 646 & $(410,889)$ & 147 & 447 & 69.2 \\
\hline Seminole, FL & 3815 & $(3658,3968)$ & 4012 & 1.05 & 197 & $(44,354)$ & 41 & 284 & 144.2 \\
\hline Sumter, FL & 2281 & $(2143,2422)$ & 2407 & 1.06 & 126 & $(-15,264)$ & 92 & 129 & 102.4 \\
\hline Volusia, FL & 7902 & $(7615,8199)$ & 8245 & 1.04 & 343 & $(46,630)$ & 61 & 446 & 130.0 \\
\hline $\begin{array}{l}\text { Appling-Jeff Davis-Long- } \\
\text { Wayne, GA }\end{array}$ & 890 & $(811,962)$ & 994 & 1.12 & 104 & $(32,183)$ & 126 & 130 & 125.0 \\
\hline $\begin{array}{l}\text { Bacon-Ben Hill-Coffee- } \\
\text { Irwin, GA }\end{array}$ & 963 & $(891,1042)$ & 1167 & 1.21 & 204 & $(125,276)$ & 255 & 196 & 96.1 \\
\hline Banks-Habersham, GA & 716 & $(655,782)$ & 798 & 1.11 & 82 & $(16,143)$ & 124 & 100 & 122.0 \\
\hline Barrow, GA & 708 & $(649,781)$ & 739 & 1.04 & 31 & $(-42,90)$ & 36 & 68 & 219.4 \\
\hline Bartow, GA & 1010 & $(933,1088)$ & 1202 & 1.19 & 192 & $(114,269)$ & 176 & 121 & 63.0 \\
\hline Bibb, GA & 1765 & $(1656,1876)$ & 2148 & 1.22 & 383 & $(272,492)$ & 250 & 208 & 54.3 \\
\hline $\begin{array}{l}\text { Bleckley-Dodge-Pulaski- } \\
\text { Twiggs, GA }\end{array}$ & 606 & $(548,662)$ & 799 & 1.32 & 193 & $(137,251)$ & 371 & 148 & 76.7 \\
\hline Brantley-Pierce-Ware, GA & 947 & $(879,1023)$ & 1144 & 1.21 & 197 & $(121,265)$ & 264 & 150 & 76.1 \\
\hline Brooks-Thomas, GA & 776 & $(705,846)$ & 862 & 1.11 & 86 & $(16,157)$ & 144 & 110 & 127.9 \\
\hline Bryan-Liberty, GA & 737 & $(678,795)$ & 818 & 1.11 & 81 & $(23,140)$ & 80 & 58 & 71.6 \\
\hline Bulloch, GA & 577 & $(524,635)$ & 647 & 1.12 & 70 & $(12,123)$ & 88 & 57 & 81.4 \\
\hline $\begin{array}{l}\text { Burke-Emanuel-Jenkins- } \\
\text { Screven, GA }\end{array}$ & 831 & $(762,899)$ & 977 & 1.18 & 146 & $(78,215)$ & 218 & 123 & 84.2 \\
\hline Butts-Lamar-Monroe, GA & 853 & $(785,928)$ & 980 & 1.15 & 127 & $(52,195)$ & 176 & 125 & 98.4 \\
\hline $\begin{array}{l}\text { Calhoun and Nearby Coun- } \\
\text { ties, GA }\end{array}$ & 899 & $(830,978)$ & 1100 & 1.22 & 201 & $(122,270)$ & 268 & 157 & 78.1 \\
\hline Camden-Charlton, GA & 536 & $(482,591)$ & 591 & 1.10 & 55 & $(0,109)$ & 80 & 42 & 76.4 \\
\hline $\begin{array}{l}\text { Candler-Evans-Tattnall- } \\
\text { Toombs, GA }\end{array}$ & 855 & $(785,929)$ & 1048 & 1.23 & 193 & $(119,263)$ & 262 & 138 & 71.5 \\
\hline Carroll-Heard, GA & 1329 & $(1243,1419)$ & 1500 & 1.13 & 171 & $(81,257)$ & 129 & 133 & 77.8 \\
\hline Catoosa, GA & 747 & $(682,810)$ & 773 & 1.03 & 26 & $(-37,91)$ & 38 & 63 & 242.3 \\
\hline Chatham, GA & 2536 & $(2411,2667)$ & 2884 & 1.14 & 348 & $(217,473)$ & 121 & 212 & 60.9 \\
\hline $\begin{array}{l}\text { Chattooga-Dade-Walker, } \\
\text { GA }\end{array}$ & 1461 & $(1366,1561)$ & 1602 & 1.10 & 141 & $(41,236)$ & 127 & 108 & 76.6 \\
\hline Cherokee-Pickens, GA & 2168 & $(2056,2275)$ & 2336 & 1.08 & 168 & $(61,280)$ & 56 & 157 & 93.5 \\
\hline
\end{tabular}


Table C1: Primary Estimates for Each County-Set

\begin{tabular}{|c|c|c|c|c|c|c|c|c|c|}
\hline County-Set & $\begin{array}{l}\text { Expected } \\
\text { Deaths }\end{array}$ & $\begin{array}{l}\text { Expected } \\
\text { Deaths } 95 \% \text { CI }\end{array}$ & $\begin{array}{l}\text { Observed } \\
\text { Deaths }\end{array}$ & $\begin{array}{l}\text { Observed/ } \\
\text { Expected }\end{array}$ & $\begin{array}{l}\text { Excess } \\
\text { Deaths }\end{array}$ & $\begin{array}{l}\text { Excess Deaths } \\
95 \% \mathrm{CI}\end{array}$ & $\begin{array}{l}\text { Excess Deaths } \\
\text { Per } 100,000\end{array}$ & $\begin{array}{l}\text { COVID-19 } \\
\text { Deaths }\end{array}$ & $\begin{array}{c}\text { COVID-19 to } \\
\text { Excess Ratio (\%) }\end{array}$ \\
\hline $\begin{array}{l}\text { Clarke-Madison-Oconee, } \\
\text { GA }\end{array}$ & 1421 & $(1331,1515)$ & 1526 & 1.07 & 105 & $(11,195)$ & 52 & 111 & 105.7 \\
\hline Clayton, GA & 1947 & $(1840,2051)$ & 2207 & 1.13 & 260 & $(156,367)$ & 87 & 213 & 81.9 \\
\hline Cobb, GA & 4704 & $(4536,4871)$ & 5362 & 1.14 & 658 & $(491,826)$ & 86 & 503 & 76.4 \\
\hline Colquitt-Mitchell, GA & 803 & $(731,879)$ & 975 & 1.21 & 172 & $(96,244)$ & 255 & 111 & 64.5 \\
\hline Columbia, GA & 1038 & $(958,1118)$ & 1211 & 1.17 & 173 & $(93,253)$ & 108 & 110 & 63.6 \\
\hline Coweta, GA & 1173 & $(1091,1257)$ & 1318 & 1.12 & 145 & $(61,227)$ & 96 & 112 & 77.2 \\
\hline $\begin{array}{l}\text { Crawford-Peach-Pike- } \\
\text { Upson, GA }\end{array}$ & 1128 & $(1039,1217)$ & 1245 & 1.10 & 117 & $(28,206)$ & 136 & 137 & 117.1 \\
\hline $\begin{array}{l}\text { Crisp-Dooly-Turner- } \\
\text { Wilcox, GA }\end{array}$ & 628 & $(565,686)$ & 823 & 1.31 & 195 & $(137,258)$ & 371 & 118 & 60.5 \\
\hline $\begin{array}{l}\text { Dawson-Lumpkin-White, } \\
\text { GA }\end{array}$ & 944 & $(868,1017)$ & 992 & 1.05 & 48 & $(-25,124)$ & 53 & 81 & 168.8 \\
\hline $\begin{array}{l}\text { Decatur-Grady-Seminole, } \\
\text { GA }\end{array}$ & 766 & $(701,833)$ & 894 & 1.17 & 128 & $(61,193)$ & 216 & 101 & 78.9 \\
\hline DeKalb, GA & 4787 & $(4618,4967)$ & 5601 & 1.17 & 814 & $(634,983)$ & 106 & 522 & 64.1 \\
\hline Dougherty, GA & 1010 & $(933,1086)$ & 1253 & 1.24 & 243 & $(167,320)$ & 273 & 199 & 81.9 \\
\hline Douglas, GA & 1063 & $(980,1138)$ & 1238 & 1.16 & 175 & $(100,258)$ & 118 & 113 & 64.6 \\
\hline Effingham, GA & 544 & $(487,597)$ & 541 & 0.99 & -3 & $(-56,54)$ & -5 & 32 & NA \\
\hline $\begin{array}{l}\text { Elbert-Franklin-Hart- } \\
\text { Stephens, GA }\end{array}$ & 1317 & $(1225,1407)$ & 1514 & 1.15 & 197 & $(107,289)$ & 207 & 147 & 74.6 \\
\hline $\begin{array}{l}\text { Fannin-Rabun-Towns- } \\
\text { Union, GA }\end{array}$ & 1159 & $(1078,1243)$ & 1357 & 1.17 & 198 & $(114,279)$ & 241 & 118 & 59.6 \\
\hline Fayette, GA & 961 & $(887,1031)$ & 1049 & 1.09 & 88 & $(18,162)$ & 77 & 74 & 84.1 \\
\hline Floyd, GA & 1200 & $(1117,1282)$ & 1347 & 1.12 & 147 & $(65,230)$ & 148 & 120 & 81.6 \\
\hline Forsyth, GA & 1337 & $(1253,1429)$ & 1415 & 1.06 & 78 & $(-14,162)$ & 31 & 81 & 103.8 \\
\hline Fulton, GA & 6999 & $(6784,7219)$ & 8201 & 1.17 & 1202 & $(982,1417)$ & 112 & 753 & 62.6 \\
\hline Gilmer-Murray, GA & 822 & $(752,893)$ & 894 & 1.09 & 72 & $(1,142)$ & 100 & 78 & 108.3 \\
\hline Glynn-McIntosh, GA & 1101 & $(1021,1187)$ & 1291 & 1.17 & 190 & $(104,270)$ & 190 & 132 & 69.5 \\
\hline Gordon, GA & 615 & $(560,676)$ & 690 & 1.12 & 75 & $(14,130)$ & 127 & 65 & 86.7 \\
\hline Gwinnett, GA & 4721 & $(4561,4887)$ & 5557 & 1.18 & 836 & $(670,996)$ & 88 & 586 & 70.1 \\
\hline Hall, GA & 1698 & $(1605,1800)$ & 2004 & 1.18 & 306 & $(204,399)$ & 147 & 220 & 71.9 \\
\hline $\begin{array}{l}\text { Hancock-Jasper-Morgan- } \\
\text { Putnam, GA }\end{array}$ & 743 & $(675,806)$ & 910 & 1.22 & 167 & $(104,235)$ & 260 & 102 & 61.1 \\
\hline Haralson-Polk, GA & 903 & $(832,976)$ & 1046 & 1.16 & 143 & $(70,214)$ & 194 & 103 & 72.0 \\
\hline $\begin{array}{l}\text { Harris-Meriwether-Talbot, } \\
\text { GA }\end{array}$ & 723 & $(664,788)$ & 765 & 1.06 & 42 & $(-23,101)$ & 66 & 57 & 135.7 \\
\hline Henry, GA & 1748 & $(1649,1841)$ & 2036 & 1.16 & 288 & $(195,387)$ & 120 & 158 & 54.9 \\
\hline Houston, GA & 1344 & $(1259,1432)$ & 1532 & 1.14 & 188 & $(100,273)$ & 118 & 118 & 62.8 \\
\hline Jackson, GA & 680 & $(621,740)$ & 787 & 1.16 & 107 & $(47,166)$ & 140 & 82 & 76.6 \\
\hline $\begin{array}{l}\text { Lanier and Nearby Coun- } \\
\text { ties, GA }\end{array}$ & 766 & $(699,832)$ & 874 & 1.14 & 108 & $(42,175)$ & 164 & 109 & 100.9 \\
\hline Lowndes, GA & 1005 & $(934,1084)$ & 1159 & 1.15 & 154 & $(75,225)$ & 129 & 111 & 72.1 \\
\hline $\begin{array}{l}\text { Marion and Nearby Coun- } \\
\text { ties, GA }\end{array}$ & 963 & $(893,1047)$ & 1012 & 1.05 & 49 & $(-35,119)$ & 58 & 137 & 279.6 \\
\hline Muscogee, GA & 2123 & $(2010,2249)$ & 2356 & 1.11 & 233 & $(107,346)$ & 120 & 218 & 93.6 \\
\hline Newton, GA & 958 & $(887,1036)$ & 1172 & 1.22 & 214 & $(136,285)$ & 190 & 117 & 54.7 \\
\hline Paulding, GA & 1099 & $(1023,1179)$ & 1215 & 1.11 & 116 & $(36,192)$ & 67 & 112 & 96.6 \\
\hline Richmond, GA & 2132 & $(2018,2249)$ & 2525 & 1.18 & 393 & $(276,507)$ & 195 & 259 & 65.9 \\
\hline Rockdale, GA & 825 & $(757,892)$ & 898 & 1.09 & 73 & $(6,141)$ & 79 & 76 & 104.1 \\
\hline Spalding, GA & 834 & $(765,903)$ & 970 & 1.16 & 136 & $(67,205)$ & 202 & 95 & 69.9 \\
\hline $\begin{array}{l}\text { Taliaferro and Nearby } \\
\text { Counties, GA }\end{array}$ & 1028 & $(952,1107)$ & 1130 & 1.10 & 102 & $(23,178)$ & 127 & 91 & 89.2 \\
\hline Tift-Worth, GA & 741 & $(676,813)$ & 800 & 1.08 & 59 & $(-13,124)$ & 97 & 122 & 206.8 \\
\hline Troup, GA & 739 & $(674,805)$ & 919 & 1.24 & 180 & $(114,245)$ & 258 & 112 & 62.2 \\
\hline Walton, GA & 964 & $(888,1041)$ & 1071 & 1.11 & 107 & $(30,183)$ & 110 & 103 & 96.3 \\
\hline
\end{tabular}


Table C1: Primary Estimates for Each County-Set

\begin{tabular}{|c|c|c|c|c|c|c|c|c|c|}
\hline County-Set & $\begin{array}{l}\text { Expected } \\
\text { Deaths }\end{array}$ & $\begin{array}{l}\text { Expected } \\
\text { Deaths } 95 \% \text { CI }\end{array}$ & $\begin{array}{l}\text { Observed } \\
\text { Deaths }\end{array}$ & $\begin{array}{l}\text { Observed/ } \\
\text { Expected }\end{array}$ & $\begin{array}{l}\text { Excess } \\
\text { Deaths }\end{array}$ & $\begin{array}{l}\text { Excess Deaths } \\
95 \% \mathrm{CI}\end{array}$ & $\begin{array}{c}\text { Excess Deaths } \\
\text { Per } 100,000\end{array}$ & $\begin{array}{l}\text { COVID-19 } \\
\text { Deaths }\end{array}$ & $\begin{array}{l}\text { COVID-19 to } \\
\text { Excess Ratio (\%) }\end{array}$ \\
\hline $\begin{array}{l}\text { Washington and Nearby } \\
\text { Counties, GA }\end{array}$ & 1565 & $(1465,1665)$ & 1835 & 1.17 & 270 & $(170,370)$ & 207 & 227 & 84.1 \\
\hline $\begin{array}{l}\text { Wheeler and Nearby Coun- } \\
\text { ties, GA }\end{array}$ & 996 & $(920,1072)$ & 1240 & 1.24 & 244 & $(168,320)$ & 279 & 189 & 77.5 \\
\hline Whitfield, GA & 902 & $(830,971)$ & 1075 & 1.19 & 173 & $(104,245)$ & 166 & 150 & 86.7 \\
\hline Hawaii (county), HI & 1924 & $(1820,2032)$ & 1864 & 0.97 & -60 & $(-168,44)$ & -29 & 54 & NA \\
\hline Honolulu, HI & 7978 & $(7740,8208)$ & 8335 & 1.04 & 357 & $(127,595)$ & 37 & 300 & 84.0 \\
\hline Kalawao-Maui, HI & 1226 & $(1138,1308)$ & 1245 & 1.02 & 19 & $(-63,107)$ & 11 & 21 & 110.5 \\
\hline Kauai, HI & 609 & $(553,668)$ & 588 & 0.97 & -21 & $(-80,35)$ & -29 & 5 & NA \\
\hline Ada, ID & 3494 & $(3349,3640)$ & 3819 & 1.09 & 325 & $(179,470)$ & 65 & 364 & 112.0 \\
\hline $\begin{array}{l}\text { Bannock-Bear Lake- } \\
\text { Caribou-Franklin, ID }\end{array}$ & 1062 & $(982,1140)$ & 1122 & 1.06 & 60 & $(-18,140)$ & 51 & 86 & 143.3 \\
\hline $\begin{array}{l}\text { Benewah-Latah-Nez Perce, } \\
\text { ID }\end{array}$ & 875 & $(806,944)$ & 916 & 1.05 & 41 & $(-28,110)$ & 45 & 56 & 136.6 \\
\hline $\begin{array}{l}\text { Bingham-Oneida-Power, } \\
\text { ID }\end{array}$ & 554 & $(497,609)$ & 590 & 1.06 & 36 & $(-19,93)$ & 61 & 54 & 150.0 \\
\hline $\begin{array}{l}\text { Boise-Gem-Payette- } \\
\text { Washington, ID }\end{array}$ & 690 & $(627,758)$ & 767 & 1.11 & 77 & $(9,140)$ & 126 & 67 & 87.0 \\
\hline $\begin{array}{l}\text { Bonner-Boundary- } \\
\text { Shoshone, ID }\end{array}$ & 801 & $(735,868)$ & 807 & 1.01 & 6 & $(-61,72)$ & 9 & 59 & 983.3 \\
\hline Bonneville, ID & 1004 & $(936,1078)$ & 1102 & 1.10 & 98 & $(24,166)$ & 81 & 121 & 123.5 \\
\hline Canyon, ID & 1752 & $(1644,1854)$ & 1921 & 1.10 & 169 & $(67,277)$ & 71 & 228 & 134.9 \\
\hline Central Idaho, ID & 689 & $(626,751)$ & 706 & 1.02 & 17 & $(-45,80)$ & 29 & 53 & 311.8 \\
\hline $\begin{array}{l}\text { Elmore-Gooding-Owyhee, } \\
\text { ID }\end{array}$ & 473 & $(424,526)$ & 572 & 1.21 & 99 & $(46,148)$ & 178 & 55 & 55.6 \\
\hline $\begin{array}{l}\text { Jefferson and Nearby } \\
\text { Counties, ID }\end{array}$ & 550 & $(494,605)$ & 606 & 1.10 & 56 & $(1,112)$ & 56 & 56 & 100.0 \\
\hline Kootenai, ID & 1667 & $(1569,1771)$ & 1662 & 1.00 & -5 & $(-109,93)$ & -3 & 128 & NA \\
\hline $\begin{array}{l}\text { Lincoln and Nearby Coun- } \\
\text { ties, ID }\end{array}$ & 773 & $(705,843)$ & 829 & 1.07 & 56 & $(-14,124)$ & 56 & 83 & 148.2 \\
\hline Twin Falls, ID & 797 & $(731,861)$ & 983 & 1.23 & 186 & $(122,252)$ & 212 & 111 & 59.7 \\
\hline Adams, IL & 827 & $(761,900)$ & 877 & 1.06 & 50 & $(-23,116)$ & 77 & 85 & 170.0 \\
\hline $\begin{array}{l}\text { Bond-Effingham-Fayette, } \\
\text { IL }\end{array}$ & 797 & $(725,863)$ & 915 & 1.15 & 118 & $(52,190)$ & 164 & 113 & 95.8 \\
\hline Boone, IL & 461 & $(414,510)$ & 488 & 1.06 & 27 & $(-22,74)$ & 50 & 65 & 240.7 \\
\hline $\begin{array}{l}\text { Brown and Nearby Coun- } \\
\text { ties, IL }\end{array}$ & 623 & $(568,683)$ & 722 & 1.16 & 99 & $(39,154)$ & 198 & 103 & 104.0 \\
\hline $\begin{array}{l}\text { Bureau-Marshall-Putnam- } \\
\text { Stark, IL }\end{array}$ & 739 & $(672,805)$ & 796 & 1.08 & 57 & $(-9,124)$ & 104 & 89 & 156.1 \\
\hline $\begin{array}{l}\text { Carroll-Jo } \\
\text { Stephenson, IL }\end{array}$ & 1019 & $(948,1099)$ & 1242 & 1.22 & 223 & $(143,294)$ & 282 & 125 & 56.1 \\
\hline Champaign, IL & 1407 & $(1323,1498)$ & 1516 & 1.08 & 109 & $(18,193)$ & 52 & 78 & 71.6 \\
\hline Christian-Shelby, IL & 686 & $(629,747)$ & 758 & 1.10 & 72 & $(11,129)$ & 134 & 100 & 138.9 \\
\hline $\begin{array}{l}\text { Clark-Crawford- } \\
\text { Cumberland-Jasper, } \\
\text { IL }\end{array}$ & 633 & $(571,691)$ & 699 & 1.10 & 66 & $(8,128)$ & 122 & 89 & 134.8 \\
\hline $\begin{array}{l}\text { Clay-Hamilton-Wayne- } \\
\text { White, IL }\end{array}$ & 704 & $(645,771)$ & 825 & 1.17 & 121 & $(54,180)$ & 238 & 119 & 98.3 \\
\hline Clinton-Marion, IL & 915 & $(844,991)$ & 1070 & 1.17 & 155 & $(79,226)$ & 208 & 141 & 91.0 \\
\hline Coles, IL & 522 & $(471,578)$ & 624 & 1.20 & 102 & $(46,153)$ & 205 & 59 & 57.8 \\
\hline Cook, IL & 41441 & $(40539,42291)$ & 52078 & 1.26 & 10637 & $(9787,11539)$ & 207 & 8046 & 75.6 \\
\hline DeKalb, IL & 764 & $(701,834)$ & 894 & 1.17 & 130 & $(60,193)$ & 124 & 68 & 52.3 \\
\hline $\begin{array}{l}\text { De Witt-Logan-Mason- } \\
\text { Menard, IL }\end{array}$ & 922 & $(852,995)$ & 1022 & 1.11 & 100 & $(27,170)$ & 143 & 121 & 121.0 \\
\hline $\begin{array}{l}\text { Douglas-Edgar-Moultrie- } \\
\text { Piatt, IL }\end{array}$ & 802 & $(726,875)$ & 918 & 1.14 & 116 & $(43,192)$ & 172 & 104 & 89.7 \\
\hline
\end{tabular}


Table C1: Primary Estimates for Each County-Set

\begin{tabular}{|c|c|c|c|c|c|c|c|c|c|}
\hline County-Set & $\begin{array}{l}\text { Expected } \\
\text { Deaths }\end{array}$ & $\begin{array}{l}\text { Expected } \\
\text { Deaths } 95 \% \text { CI }\end{array}$ & $\begin{array}{l}\text { Observed } \\
\text { Deaths }\end{array}$ & $\begin{array}{l}\text { Observed/ } \\
\text { Expected }\end{array}$ & $\begin{array}{l}\text { Excess } \\
\text { Deaths }\end{array}$ & $\begin{array}{l}\text { Excess Deaths } \\
95 \% \text { CI }\end{array}$ & $\begin{array}{l}\text { Excess Deaths } \\
\text { Per } 100,000\end{array}$ & $\begin{array}{l}\text { COVID-19 } \\
\text { Deaths }\end{array}$ & $\begin{array}{c}\text { COVID-19 to } \\
\text { Excess Ratio (\%) }\end{array}$ \\
\hline DuPage, IL & 6516 & $(6309,6730)$ & 7618 & 1.17 & 1102 & $(888,1309)$ & 119 & 881 & 79.9 \\
\hline $\begin{array}{l}\text { Edwards-Lawrence- } \\
\text { Richland-Wabash, IL }\end{array}$ & 610 & $(554,669)$ & 732 & 1.20 & 122 & $(63,178)$ & 251 & 93 & 76.2 \\
\hline $\begin{array}{l}\text { Ford-Iroquois-Livingston, } \\
\text { IL }\end{array}$ & 1072 & $(987,1151)$ & 1187 & 1.11 & 115 & $(36,200)$ & 153 & 141 & 122.6 \\
\hline $\begin{array}{l}\text { Franklin-Gallatin-Saline, } \\
\text { IL }\end{array}$ & 972 & $(895,1049)$ & 1101 & 1.13 & 129 & $(52,206)$ & 193 & 119 & 92.2 \\
\hline Fulton-McDonough, IL & 831 & $(765,906)$ & 916 & 1.10 & 85 & $(10,151)$ & 133 & 87 & 102.4 \\
\hline Greene-Jersey-Morgan, IL & 886 & $(813,956)$ & 1008 & 1.14 & 122 & $(52,195)$ & 179 & 154 & 126.2 \\
\hline Grundy, IL & 440 & $(391,491)$ & 545 & 1.24 & 105 & $(54,154)$ & 203 & 49 & 46.7 \\
\hline $\begin{array}{l}\text { Hancock-Henderson- } \\
\text { Mercer-Warren, IL }\end{array}$ & 707 & $(638,771)$ & 858 & 1.21 & 151 & $(87,220)$ & 269 & 104 & 68.9 \\
\hline Henry, IL & 548 & $(493,605)$ & 689 & 1.26 & 141 & $(84,196)$ & 289 & 70 & 49.6 \\
\hline Jackson, IL & 491 & $(441,543)$ & 614 & 1.25 & 123 & $(71,173)$ & 218 & 74 & 60.2 \\
\hline Jefferson-Washington, IL & 615 & $(560,671)$ & 742 & 1.21 & 127 & $(71,182)$ & 248 & 100 & 78.7 \\
\hline Kane, IL & 3497 & $(3361,3646)$ & 4186 & 1.20 & 689 & $(540,825)$ & 128 & 558 & 81.0 \\
\hline Kankakee, IL & 1229 & $(1143,1318)$ & 1353 & 1.10 & 124 & $(35,210)$ & 114 & 142 & 114.5 \\
\hline Kendall, IL & 644 & $(585,700)$ & 769 & 1.19 & 125 & $(69,184)$ & 95 & 78 & 62.4 \\
\hline Knox, IL & 681 & $(617,745)$ & 816 & 1.20 & 135 & $(71,199)$ & 276 & 115 & 85.2 \\
\hline Lake, IL & 4934 & $(4762,5109)$ & 5735 & 1.16 & 801 & $(626,973)$ & 115 & 749 & 93.5 \\
\hline La Salle, IL & 1361 & $(1268,1455)$ & 1524 & 1.12 & 163 & $(69,256)$ & 150 & 207 & 127.0 \\
\hline Lee-Whiteside, IL & 1092 & $(1012,1172)$ & 1278 & 1.17 & 186 & $(106,266)$ & 210 & 234 & 125.8 \\
\hline McHenry, IL & 2282 & $(2164,2403)$ & 2575 & 1.13 & 293 & $(172,411)$ & 95 & 251 & 85.7 \\
\hline McLean, IL & 1287 & $(1204,1371)$ & 1428 & 1.11 & 141 & $(57,224)$ & 82 & 131 & 92.9 \\
\hline Macon, IL & 1295 & $(1200,1378)$ & 1448 & 1.12 & 153 & $(70,248)$ & 148 & 159 & 103.9 \\
\hline Macoupin-Montgomery, IL & 949 & $(876,1031)$ & 1086 & 1.14 & 137 & $(55,210)$ & 187 & 102 & 74.5 \\
\hline Madison, IL & 3051 & $(2907,3192)$ & 3385 & 1.11 & 334 & $(193,478)$ & 127 & 330 & 98.8 \\
\hline Monroe-St. Clair, IL & 3020 & $(2881,3159)$ & 3541 & 1.17 & 521 & $(382,660)$ & 178 & 398 & 76.4 \\
\hline Ogle, IL & 559 & $(506,612)$ & 611 & 1.09 & 52 & $(-1,105)$ & 103 & 63 & 121.2 \\
\hline Peoria-Woodford, IL & 2252 & $(2133,2384)$ & 2555 & 1.13 & 303 & $(171,422)$ & 141 & 280 & 92.4 \\
\hline Perry-Randolph, IL & 622 & $(565,686)$ & 698 & 1.12 & 76 & $(12,133)$ & 144 & 91 & 119.7 \\
\hline Rock Island, IL & 1645 & $(1540,1746)$ & 1949 & 1.18 & 304 & $(203,409)$ & 214 & 230 & 75.7 \\
\hline Sangamon, IL & 2063 & $(1948,2173)$ & 2226 & 1.08 & 163 & $(53,278)$ & 85 & 204 & 125.2 \\
\hline Southern Illinois, IL & 886 & $(811,961)$ & 1007 & 1.14 & 121 & $(46,196)$ & 197 & 110 & 90.9 \\
\hline Tazewell, IL & 1429 & $(1338,1525)$ & 1674 & 1.17 & 245 & $(149,336)$ & 188 & 202 & 82.4 \\
\hline Vermilion, IL & 991 & $(912,1074)$ & 1104 & 1.11 & 113 & $(30,192)$ & 151 & 94 & 83.2 \\
\hline Will, IL & 5104 & $(4935,5298)$ & 5892 & 1.15 & 788 & $(594,957)$ & 113 & 701 & 89.0 \\
\hline Williamson, IL & 761 & $(694,830)$ & 872 & 1.15 & 111 & $(42,178)$ & 166 & 91 & 82.0 \\
\hline Winnebago, IL & 3179 & $(3029,3328)$ & 3486 & 1.10 & 307 & $(158,457)$ & 108 & 371 & 120.8 \\
\hline Adams-Wells, IN & 654 & $(592,717)$ & 776 & 1.19 & 122 & $(59,184)$ & 188 & 96 & 78.7 \\
\hline Allen, IN & 3650 & $(3495,3812)$ & 4051 & 1.11 & 401 & $(239,556)$ & 105 & 482 & 120.2 \\
\hline Bartholomew-Brown, IN & 944 & $(869,1023)$ & 1075 & 1.14 & 131 & $(52,206)$ & 133 & 137 & 104.6 \\
\hline Benton-Jasper-Newton, IN & 606 & $(545,671)$ & 734 & 1.21 & 128 & $(63,189)$ & 230 & 78 & 60.9 \\
\hline $\begin{array}{l}\text { Blackford-Jay-Randolph, } \\
\text { IN }\end{array}$ & 743 & $(678,808)$ & 845 & 1.14 & 102 & $(37,167)$ & 180 & 120 & 117.6 \\
\hline Boone, IN & 570 & $(520,623)$ & 614 & 1.08 & 44 & $(-9,94)$ & 63 & 86 & 195.5 \\
\hline Carroll-Clinton, IN & 576 & $(520,634)$ & 652 & 1.13 & 76 & $(18,132)$ & 145 & 58 & 76.3 \\
\hline Cass-White, IN & 779 & $(707,852)$ & 850 & 1.09 & 71 & $(-2,143)$ & 114 & 115 & 162.0 \\
\hline Clark-Scott, IN & 1711 & $(1599,1809)$ & 1869 & 1.09 & 158 & $(60,270)$ & 111 & 184 & 116.5 \\
\hline $\begin{array}{l}\text { Clay-Sullivan-Vermillion- } \\
\text { Vigo, IN }\end{array}$ & 1963 & $(1853,2071)$ & 2228 & 1.13 & 265 & $(157,375)$ & 157 & 250 & 94.3 \\
\hline $\begin{array}{l}\text { Crawford-Harrison-Perry, } \\
\text { IN }\end{array}$ & 810 & $(742,878)$ & 864 & 1.07 & 54 & $(-14,122)$ & 76 & 84 & 155.6 \\
\hline Daviess-Knox-Martin, IN & 914 & $(840,987)$ & 1076 & 1.18 & 162 & $(89,236)$ & 203 & 169 & 104.3 \\
\hline $\begin{array}{l}\text { Dearborn-Ohio-Ripley- } \\
\text { Switzerland, IN }\end{array}$ & 1044 & $(962,1118)$ & 1122 & 1.07 & 78 & $(4,160)$ & 82 & 128 & 164.1 \\
\hline
\end{tabular}


Table C1: Primary Estimates for Each County-Set

\begin{tabular}{|c|c|c|c|c|c|c|c|c|c|}
\hline County-Set & $\begin{array}{l}\text { Expected } \\
\text { Deaths }\end{array}$ & $\begin{array}{l}\text { Expected } \\
\text { Deaths } 95 \% \text { CI }\end{array}$ & $\begin{array}{l}\text { Observed } \\
\text { Deaths }\end{array}$ & $\begin{array}{l}\text { Observed/ } \\
\text { Expected }\end{array}$ & $\begin{array}{l}\text { Excess } \\
\text { Deaths }\end{array}$ & $\begin{array}{l}\text { Excess Deaths } \\
95 \% \mathrm{CI}\end{array}$ & $\begin{array}{l}\text { Excess Deaths } \\
\text { Per } 100,000\end{array}$ & $\begin{array}{l}\text { COVID-19 } \\
\text { Deaths }\end{array}$ & $\begin{array}{c}\text { COVID-19 to } \\
\text { Excess Ratio (\%) }\end{array}$ \\
\hline Decatur-Shelby, IN & 810 & $(740,876)$ & 958 & 1.18 & 148 & $(82,218)$ & 205 & 132 & 89.2 \\
\hline DeKalb-Steuben, IN & 812 & $(743,876)$ & 913 & 1.12 & 101 & $(37,170)$ & 128 & 103 & 102.0 \\
\hline Delaware, IN & 1400 & $(1307,1492)$ & 1595 & 1.14 & 195 & $(103,288)$ & 172 & 141 & 72.3 \\
\hline Dubois-Pike-Spencer, IN & 807 & $(740,876)$ & 919 & 1.14 & 112 & $(43,179)$ & 149 & 100 & 89.3 \\
\hline Elkhart, IN & 1809 & $(1703,1908)$ & 2093 & 1.16 & 284 & $(185,390)$ & 137 & 349 & 122.9 \\
\hline $\begin{array}{l}\text { Fayette-Franklin-Union, } \\
\text { IN }\end{array}$ & 635 & $(574,691)$ & 787 & 1.24 & 152 & $(96,213)$ & 291 & 84 & 55.3 \\
\hline Floyd, IN & 883 & $(815,950)$ & 967 & 1.10 & 84 & $(17,152)$ & 106 & 107 & 127.4 \\
\hline $\begin{array}{l}\text { Fountain-Montgomery- } \\
\text { Warren, IN }\end{array}$ & 750 & $(686,821)$ & 861 & 1.15 & 111 & $(40,175)$ & 176 & 98 & 88.3 \\
\hline Fulton-Miami-Wabash, IN & 1120 & $(1044,1207)$ & 1268 & 1.13 & 148 & $(61,224)$ & 171 & 138 & 93.2 \\
\hline Gibson-Posey, IN & 656 & $(598,714)$ & 714 & 1.09 & 58 & $(0,116)$ & 99 & 95 & 163.8 \\
\hline Grant, IN & 900 & $(828,979)$ & 1052 & 1.17 & 152 & $(73,224)$ & 233 & 129 & 84.9 \\
\hline $\begin{array}{l}\text { Greene-Owen-Parke- } \\
\text { Putnam, IN }\end{array}$ & 1219 & $(1136,1308)$ & 1371 & 1.12 & 152 & $(63,235)$ & 141 & 154 & 101.3 \\
\hline Hamilton, IN & 2040 & $(1938,2138)$ & 2353 & 1.15 & 313 & $(215,415)$ & 91 & 305 & 97.4 \\
\hline Hancock, IN & 715 & $(647,784)$ & 764 & 1.07 & 49 & $(-20,117)$ & 62 & 85 & 173.5 \\
\hline Hendricks, IN & 1360 & $(1269,1453)$ & 1535 & 1.13 & 175 & $(82,266)$ & 101 & 205 & 117.1 \\
\hline Henry-Rush, IN & 833 & $(768,904)$ & 931 & 1.12 & 98 & $(27,163)$ & 151 & 87 & 88.8 \\
\hline Howard-Tipton, IN & 1358 & $(1255,1451)$ & 1490 & 1.10 & 132 & $(39,235)$ & 135 & 190 & 143.9 \\
\hline Huntington-Whitley, IN & 758 & $(692,827)$ & 884 & 1.17 & 126 & $(57,192)$ & 178 & 75 & 59.5 \\
\hline Jackson-Washington, IN & 836 & $(770,908)$ & 902 & 1.08 & 66 & $(-6,132)$ & 90 & 78 & 118.2 \\
\hline Jennings-Jefferson, IN & 738 & $(674,807)$ & 805 & 1.09 & 67 & $(-2,131)$ & 112 & 87 & 129.9 \\
\hline Johnson, IN & 1451 & $(1360,1549)$ & 1696 & 1.17 & 245 & $(147,336)$ & 152 & 240 & 98.0 \\
\hline Kosciusko, IN & 758 & $(692,822)$ & 840 & 1.11 & 82 & $(18,148)$ & 102 & 76 & 92.7 \\
\hline LaGrange-Noble, IN & 767 & $(700,833)$ & 872 & 1.14 & 105 & $(39,172)$ & 121 & 131 & 124.8 \\
\hline Lake, IN & 5310 & $(5125,5515)$ & 6148 & 1.16 & 838 & $(633,1023)$ & 173 & 695 & 82.9 \\
\hline LaPorte, IN & 1281 & $(1187,1370)$ & 1473 & 1.15 & 192 & $(103,286)$ & 175 & 140 & 72.9 \\
\hline Lawrence-Orange, IN & 818 & $(747,893)$ & 955 & 1.17 & 137 & $(62,208)$ & 210 & 125 & 91.2 \\
\hline Madison, IN & 1569 & $(1473,1670)$ & 1825 & 1.16 & 256 & $(155,352)$ & 198 & 242 & 94.5 \\
\hline Marion, IN & 8599 & $(8333,8864)$ & 10262 & 1.19 & 1663 & $(1398,1929)$ & 172 & 1356 & 81.5 \\
\hline $\begin{array}{l}\text { Marshall-Pulaski-Starke, } \\
\text { IN }\end{array}$ & 922 & $(850,997)$ & 1138 & 1.23 & 216 & $(141,288)$ & 266 & 168 & 77.8 \\
\hline Monroe, IN & 1019 & $(947,1096)$ & 1209 & 1.19 & 190 & $(113,262)$ & 129 & 121 & 63.7 \\
\hline Morgan, IN & 776 & $(708,841)$ & 854 & 1.10 & 78 & $(13,146)$ & 110 & 98 & 125.6 \\
\hline Porter, IN & 1660 & $(1557,1757)$ & 1861 & 1.12 & 201 & $(104,304)$ & 117 & 200 & 99.5 \\
\hline St. Joseph, IN & 2875 & $(2733,3009)$ & 3294 & 1.15 & 419 & $(285,561)$ & 154 & 417 & 99.5 \\
\hline Tippecanoe, IN & 1281 & $(1195,1366)$ & 1377 & 1.07 & 96 & $(11,182)$ & 49 & 100 & 104.2 \\
\hline Vanderburgh, IN & 2081 & $(1966,2205)$ & 2405 & 1.16 & 324 & $(200,439)$ & 179 & 261 & 80.6 \\
\hline Warrick, IN & 662 & $(602,728)$ & 797 & 1.20 & 135 & $(69,195)$ & 215 & 95 & 70.4 \\
\hline Wayne, IN & 980 & $(900,1059)$ & 1093 & 1.12 & 113 & $(34,193)$ & 172 & 143 & 126.5 \\
\hline $\begin{array}{l}\text { Allamakee-Clayton- } \\
\text { Winneshiek, IA }\end{array}$ & 595 & $(540,658)$ & 672 & 1.13 & 77 & $(14,132)$ & 151 & 109 & 141.6 \\
\hline $\begin{array}{l}\text { Audubon and Nearby } \\
\text { Counties, IA }\end{array}$ & 688 & $(624,757)$ & 775 & 1.13 & 87 & $(18,151)$ & 183 & 123 & 141.4 \\
\hline Benton-Iowa (county)- & 852 & $(782,928)$ & 997 & 1.17 & 145 & $(69,215)$ & 196 & 134 & 92.4 \\
\hline Keokuk-Washington, IA & & & & & & & & & \\
\hline Black Hawk, IA & 1303 & $(1216,1396)$ & 1505 & 1.16 & 202 & $(109,289)$ & 153 & 255 & 126.2 \\
\hline Boone-Carroll-Greene, IA & 658 & $(600,718)$ & 725 & 1.10 & 67 & $(7,125)$ & 121 & 64 & 95.5 \\
\hline Bremer-Butler-Grundy, IA & 613 & $(555,679)$ & 676 & 1.10 & 63 & $(-3,121)$ & 121 & 105 & 166.7 \\
\hline $\begin{array}{l}\text { Buchanan-Delaware- } \\
\text { Fayette, IA }\end{array}$ & 652 & $(593,716)$ & 752 & 1.15 & 100 & $(36,159)$ & 174 & 88 & 88.0 \\
\hline $\begin{array}{l}\text { Cedar-Clinton-Jackson- } \\
\text { Jones, IA }\end{array}$ & 1202 & $(1115,1291)$ & 1329 & 1.11 & 127 & $(38,214)$ & 121 & 183 & 144.1 \\
\hline $\begin{array}{l}\text { Cerro Gordo-Hancock- } \\
\text { Winnebago, IA }\end{array}$ & 792 & $(720,861)$ & 926 & 1.17 & 134 & $(65,206)$ & 213 & 133 & 99.3 \\
\hline
\end{tabular}


Table C1: Primary Estimates for Each County-Set

\begin{tabular}{|c|c|c|c|c|c|c|c|c|c|}
\hline County-Set & $\begin{array}{l}\text { Expected } \\
\text { Deaths }\end{array}$ & $\begin{array}{l}\text { Expected } \\
\text { Deaths } 95 \% \text { CI }\end{array}$ & $\begin{array}{l}\text { Observed } \\
\text { Deaths }\end{array}$ & $\begin{array}{l}\text { Observed/ } \\
\text { Expected }\end{array}$ & $\begin{array}{l}\text { Excess } \\
\text { Deaths }\end{array}$ & $\begin{array}{l}\text { Excess Deaths } \\
95 \% \text { CI }\end{array}$ & $\begin{array}{l}\text { Excess Deaths } \\
\text { Per } 100,000\end{array}$ & $\begin{array}{l}\text { COVID-19 } \\
\text { Deaths }\end{array}$ & $\begin{array}{c}\text { COVID-19 to } \\
\text { Excess Ratio (\%) }\end{array}$ \\
\hline Dallas, IA & 570 & $(516,628)$ & 637 & 1.12 & 67 & $(9,121)$ & 69 & 74 & 110.4 \\
\hline $\begin{array}{l}\text { Davis-Jefferson-Van } \\
\text { Buren-Wapello, IA }\end{array}$ & 817 & $(742,887)$ & 927 & 1.13 & 110 & $(40,185)$ & 157 & 162 & 147.3 \\
\hline Des Moines-Henry-Lee, IA & 1127 & $(1049,1208)$ & 1309 & 1.16 & 182 & $(101,260)$ & 197 & 120 & 65.9 \\
\hline Dubuque, IA & 935 & $(861,1007)$ & 1034 & 1.11 & 99 & $(27,173)$ & 103 & 161 & 162.6 \\
\hline $\begin{array}{l}\text { Franklin-Hamilton- } \\
\text { Hardin-Wright, IA }\end{array}$ & 713 & $(647,778)$ & 816 & 1.14 & 103 & $(38,169)$ & 190 & 112 & 108.7 \\
\hline $\begin{array}{l}\text { Fremont-Harrison-Mills- } \\
\text { Pottawattamie, IA }\end{array}$ & 1410 & $(1318,1506)$ & 1694 & 1.20 & 284 & $(188,376)$ & 219 & 212 & 74.6 \\
\hline Jasper-Poweshiek, IA & 631 & $(575,693)$ & 708 & 1.12 & 77 & $(15,133)$ & 137 & 89 & 115.6 \\
\hline Johnson, IA & 802 & $(738,868)$ & 856 & 1.07 & 54 & $(-12,118)$ & 35 & 58 & 107.4 \\
\hline Linn, IA & 1893 & $(1791,2000)$ & 2269 & 1.20 & 376 & $(269,478)$ & 164 & 292 & 77.7 \\
\hline Louisa-Muscatine, IA & 547 & $(495,605)$ & 636 & 1.16 & 89 & $(31,141)$ & 164 & 121 & 136.0 \\
\hline $\begin{array}{l}\text { Lucas and Nearby Coun- } \\
\text { ties, IA }\end{array}$ & 682 & $(622,745)$ & 770 & 1.13 & 88 & $(25,148)$ & 166 & 105 & 119.3 \\
\hline $\begin{array}{l}\text { Lyon-O'Brien-Osceola- } \\
\text { Sioux, IA }\end{array}$ & 638 & $(578,700)$ & 792 & 1.24 & 154 & $(92,214)$ & 231 & 173 & 112.3 \\
\hline Madison-Warren, IA & 639 & $(585,702)$ & 659 & 1.03 & 20 & $(-43,74)$ & 29 & 61 & 305.0 \\
\hline Mahaska-Marion, IA & 606 & $(551,666)$ & 678 & 1.12 & 72 & $(12,127)$ & 130 & 100 & 138.9 \\
\hline Marshall-Tama, IA & 708 & $(647,779)$ & 824 & 1.16 & 116 & $(45,177)$ & 206 & 123 & 106.0 \\
\hline $\begin{array}{l}\text { Mitchell and Nearby Coun- } \\
\text { ties, IA }\end{array}$ & 662 & $(603,723)$ & 771 & 1.16 & 109 & $(48,168)$ & 200 & 113 & 103.7 \\
\hline $\begin{array}{l}\text { Palo Alto and Nearby } \\
\text { Counties, IA }\end{array}$ & 818 & $(749,885)$ & 966 & 1.18 & 148 & $(81,217)$ & 226 & 146 & 98.6 \\
\hline $\begin{array}{l}\text { Pocahontas and Nearby } \\
\text { Counties, IA }\end{array}$ & 1073 & $(994,1148)$ & 1291 & 1.20 & 218 & $(143,297)$ & 242 & 172 & 78.9 \\
\hline Polk, IA & 3872 & $(3714,4038)$ & 4459 & 1.15 & 587 & $(421,745)$ & 118 & 484 & 82.5 \\
\hline Scott, IA & 1607 & $(1515,1708)$ & 1910 & 1.19 & 303 & $(202,395)$ & 174 & 189 & 62.4 \\
\hline Story, IA & 611 & $(554,669)$ & 563 & 0.92 & -48 & $(-106,9)$ & -48 & 40 & NA \\
\hline $\begin{array}{l}\text { Taylor and Nearby Coun- } \\
\text { ties, IA }\end{array}$ & 708 & $(646,776)$ & 828 & 1.17 & 120 & $(52,182)$ & 231 & 98 & 81.7 \\
\hline West Central Iowa, IA & 1883 & $(1768,1992)$ & 2222 & 1.18 & 339 & $(230,454)$ & 197 & 366 & 108.0 \\
\hline $\begin{array}{l}\text { Allen-Anderson-Franklin- } \\
\text { Osage, KS }\end{array}$ & 755 & $(690,820)$ & 848 & 1.12 & 93 & $(28,158)$ & 150 & 58 & 62.4 \\
\hline Bourbon-Linn-Miami, KS & 646 & $(588,707)$ & 699 & 1.08 & 53 & $(-8,111)$ & 91 & 46 & 86.8 \\
\hline $\begin{array}{l}\text { Brown and Nearby Coun- } \\
\text { ties, KS }\end{array}$ & 1035 & $(956,1112)$ & 1129 & 1.09 & 94 & $(17,173)$ & 110 & 151 & 160.6 \\
\hline Butler-Harvey, KS & 1101 & $(1024,1179)$ & 1222 & 1.11 & 121 & $(43,198)$ & 120 & 87 & 71.9 \\
\hline Central Kansas, KS & 1206 & $(1121,1293)$ & 1488 & 1.23 & 282 & $(195,367)$ & 270 & 199 & 70.6 \\
\hline $\begin{array}{l}\text { Chase-Coffey-Lyon- } \\
\text { Marion, KS }\end{array}$ & 599 & $(541,658)$ & 658 & 1.10 & 59 & $(0,117)$ & 106 & 97 & 164.4 \\
\hline Cherokee-Crawford, KS & 732 & $(672,797)$ & 895 & 1.22 & 163 & $(98,223)$ & 277 & 87 & 53.4 \\
\hline $\begin{array}{l}\text { Cloud and Nearby Coun- } \\
\text { ties, KS }\end{array}$ & 749 & $(680,812)$ & 801 & 1.07 & 52 & $(-11,121)$ & 102 & 97 & 186.5 \\
\hline $\begin{array}{l}\text { Dickinson-Geary-Morris, } \\
\text { KS }\end{array}$ & 512 & $(462,566)$ & 582 & 1.14 & 70 & $(16,120)$ & 127 & 68 & 97.1 \\
\hline Douglas, KS & 801 & $(741,866)$ & 872 & 1.09 & 71 & $(6,131)$ & 58 & 63 & 88.7 \\
\hline $\begin{array}{l}\text { Elk and Nearby Counties, } \\
\text { KS }\end{array}$ & 809 & $(741,880)$ & 860 & 1.06 & 51 & $(-20,119)$ & 87 & 100 & 196.1 \\
\hline Johnson, KS & 4323 & $(4155,4493)$ & 4722 & 1.09 & 399 & $(229,567)$ & 65 & 512 & 128.3 \\
\hline $\begin{array}{l}\text { Labette-Montgomery- } \\
\text { Neosho, KS }\end{array}$ & 925 & $(852,1004)$ & 1001 & 1.08 & 76 & $(-3,149)$ & 114 & 96 & 126.3 \\
\hline Leavenworth, KS & 672 & $(608,734)$ & 795 & 1.18 & 123 & $(61,187)$ & 150 & 65 & 52.8 \\
\hline Northwest Kansas, KS & 684 & $(622,750)$ & 768 & 1.12 & 84 & $(18,146)$ & 164 & 143 & 170.2 \\
\hline Pawnee and Nearby Coun- & 640 & $(581,699)$ & 740 & 1.16 & 100 & $(41,159)$ & 178 & 115 & 115.0 \\
\hline
\end{tabular}


Table C1: Primary Estimates for Each County-Set

\begin{tabular}{|c|c|c|c|c|c|c|c|c|c|}
\hline County-Set & $\begin{array}{l}\text { Expected } \\
\text { Deaths }\end{array}$ & $\begin{array}{l}\text { Expected } \\
\text { Deaths } 95 \% \text { CI }\end{array}$ & $\begin{array}{l}\text { Observed } \\
\text { Deaths }\end{array}$ & $\begin{array}{l}\text { Observed/ } \\
\text { Expected }\end{array}$ & $\begin{array}{l}\text { Excess } \\
\text { Deaths }\end{array}$ & $\begin{array}{l}\text { Excess Deaths } \\
95 \% \mathrm{CI}\end{array}$ & $\begin{array}{l}\text { Excess Deaths } \\
\text { Per } 100,000\end{array}$ & $\begin{array}{l}\text { COVID-19 } \\
\text { Deaths }\end{array}$ & $\begin{array}{l}\text { COVID-19 to } \\
\text { Excess Ratio (\%) }\end{array}$ \\
\hline $\begin{array}{l}\text { Pottawatomie-Riley- } \\
\text { Wabaunsee, KS }\end{array}$ & 653 & $(598,712)$ & 684 & 1.05 & 31 & $(-28,86)$ & 30 & 44 & 141.9 \\
\hline Reno, KS & 730 & $(666,797)$ & 867 & 1.19 & 137 & $(70,201)$ & 223 & 107 & 78.1 \\
\hline Saline, KS & 588 & $(532,647)$ & 655 & 1.11 & 67 & $(8,123)$ & 124 & 84 & 125.4 \\
\hline $\begin{array}{l}\text { Scott and Nearby Coun- } \\
\text { ties, KS }\end{array}$ & 415 & $(369,464)$ & 541 & 1.30 & 126 & $(77,172)$ & 242 & 107 & 84.9 \\
\hline Sedgwick, KS & 4744 & $(4572,4927)$ & 5735 & 1.21 & 991 & $(808,1163)$ & 193 & 550 & 55.5 \\
\hline Shawnee, KS & 2059 & $(1940,2175)$ & 2289 & 1.11 & 230 & $(114,349)$ & 131 & 262 & 113.9 \\
\hline South Central Kansas, KS & 808 & $(741,878)$ & 960 & 1.19 & 152 & $(82,219)$ & 287 & 130 & 85.5 \\
\hline Southwest Kansas, KS & 442 & $(393,490)$ & 511 & 1.16 & 69 & $(21,118)$ & 132 & 93 & 134.8 \\
\hline Wyandotte, KS & 1413 & $(1324,1499)$ & 1723 & 1.22 & 310 & $(224,399)$ & 187 & 227 & 73.2 \\
\hline $\begin{array}{l}\text { Adair and Nearby Coun- } \\
\text { ties, KY }\end{array}$ & 1180 & $(1096,1266)$ & 1400 & 1.19 & 220 & $(134,304)$ & 245 & 162 & 73.6 \\
\hline Anderson-Franklin, KY & 888 & $(820,957)$ & 921 & 1.04 & 33 & $(-36,101)$ & 44 & 57 & 172.7 \\
\hline $\begin{array}{l}\text { Barren and Nearby Coun- } \\
\text { ties, KY }\end{array}$ & 1380 & $(1283,1471)$ & 1496 & 1.08 & 116 & $(25,213)$ & 109 & 180 & 155.2 \\
\hline $\begin{array}{l}\text { Bath-Clark-Montgomery- } \\
\text { Rowan, KY }\end{array}$ & 1224 & $(1137,1303)$ & 1256 & 1.03 & 32 & $(-47,119)$ & 31 & 60 & 187.5 \\
\hline Bell-Harlan, KY & 868 & $(792,946)$ & 1000 & 1.15 & 132 & $(54,208)$ & 255 & 79 & 59.8 \\
\hline Boone, KY & 1034 & $(958,1111)$ & 1088 & 1.05 & 54 & $(-23,130)$ & 41 & 93 & 172.2 \\
\hline $\begin{array}{l}\text { Bourbon-Fleming-Lewis- } \\
\text { Nicholas, KY }\end{array}$ & 745 & $(679,811)$ & 767 & 1.03 & 22 & $(-44,88)$ & 41 & 60 & 272.7 \\
\hline Boyd-Carter-Greenup, KY & 1574 & $(1471,1682)$ & 1701 & 1.08 & 127 & $(19,230)$ & 117 & 125 & 98.4 \\
\hline Boyle-Lincoln-Mercer, KY & 1015 & $(936,1099)$ & 1097 & 1.08 & 82 & $(-2,161)$ & 106 & 118 & 143.9 \\
\hline $\begin{array}{l}\text { Bracken and Nearby Coun- } \\
\text { ties, KY }\end{array}$ & 795 & $(725,863)$ & 844 & 1.06 & 49 & $(-19,119)$ & 80 & 50 & 102.0 \\
\hline $\begin{array}{l}\text { Breckinridge-Grayson- } \\
\text { Hancock-Meade, KY }\end{array}$ & 997 & $(920,1077)$ & 1086 & 1.09 & 89 & $(9,166)$ & 104 & 80 & 89.9 \\
\hline Bullitt-Spencer, KY & 914 & $(844,989)$ & 1000 & 1.09 & 86 & $(11,156)$ & 84 & 86 & 100.0 \\
\hline $\begin{array}{l}\text { Butler-McLean- } \\
\text { Muhlenberg-Ohio, KY }\end{array}$ & 1009 & $(932,1087)$ & 1097 & 1.09 & 88 & $(10,165)$ & 115 & 112 & 127.3 \\
\hline Calloway-Marshall, KY & 881 & $(806,959)$ & 930 & 1.06 & 49 & $(-29,124)$ & 69 & 79 & 161.2 \\
\hline Campbell, KY & 915 & $(848,993)$ & 968 & 1.06 & 53 & $(-25,120)$ & 56 & 65 & 122.6 \\
\hline Christian, KY & 659 & $(600,717)$ & 788 & 1.20 & 129 & $(71,188)$ & 177 & 73 & 56.6 \\
\hline $\begin{array}{l}\text { Clay-Jackson-Leslie- } \\
\text { Owsley, KY }\end{array}$ & 702 & $(637,768)$ & 753 & 1.07 & 51 & $(-15,116)$ & 108 & 62 & 121.6 \\
\hline $\begin{array}{l}\text { Clinton-Cumberland- } \\
\text { McCreary-Wayne, KY }\end{array}$ & 742 & $(677,812)$ & 823 & 1.11 & 81 & $(11,146)$ & 149 & 65 & 80.2 \\
\hline Daviess, KY & 1164 & $(1082,1249)$ & 1214 & 1.04 & 50 & $(-35,132)$ & 49 & 104 & 208.0 \\
\hline Edmonson-Warren, KY & 1273 & $(1185,1362)$ & 1454 & 1.14 & 181 & $(92,269)$ & 122 & 128 & 70.7 \\
\hline $\begin{array}{l}\text { Elliott-Johnson-Lawrence- } \\
\text { Magoffin, KY }\end{array}$ & 835 & $(767,906)$ & 821 & 0.98 & -14 & $(-85,54)$ & -25 & 55 & NA \\
\hline $\begin{array}{l}\text { Estill-Madison-Rockcastle, } \\
\text { KY }\end{array}$ & 1310 & $(1222,1407)$ & 1432 & 1.09 & 122 & $(25,210)$ & 97 & 97 & 79.5 \\
\hline Fayette, KY & 2619 & $(2489,2747)$ & 2906 & 1.11 & 287 & $(159,417)$ & 88 & 168 & 58.5 \\
\hline Floyd-Martin, KY & 705 & $(637,771)$ & 823 & 1.17 & 118 & $(52,186)$ & 256 & 59 & 50.0 \\
\hline Garrard-Jessamine, KY & 750 & $(693,812)$ & 783 & 1.04 & 33 & $(-29,90)$ & 45 & 78 & 236.4 \\
\hline Hardin-Larue, KY & 1242 & $(1162,1324)$ & 1393 & 1.12 & 151 & $(69,231)$ & 117 & 108 & 71.5 \\
\hline Henderson-Union, KY & 731 & $(664,800)$ & 801 & 1.10 & 70 & $(1,137)$ & 119 & 78 & 111.4 \\
\hline Henry-Shelby, KY & 634 & $(575,692)$ & 682 & 1.08 & 48 & $(-10,107)$ & 72 & 60 & 125.0 \\
\hline Hopkins-Webster, KY & 793 & $(723,861)$ & 922 & 1.16 & 129 & $(61,199)$ & 224 & 128 & 99.2 \\
\hline Jefferson, KY & 8416 & $(8130,8700)$ & 9561 & 1.14 & 1145 & $(861,1431)$ & 149 & 838 & 73.2 \\
\hline Kenton, KY & 1622 & $(1527,1723)$ & 1642 & 1.01 & 20 & $(-81,115)$ & 12 & 119 & 595.0 \\
\hline Knott-Letcher-Perry, KY & 996 & $(922,1074)$ & 1127 & 1.13 & 131 & $(53,205)$ & 215 & 74 & 56.5 \\
\hline Knox-Whitley, KY & 972 & $(891,1048)$ & 1037 & 1.07 & 65 & $(-11,146)$ & 96 & 71 & 109.2 \\
\hline Laurel, KY & 720 & $(653,788)$ & 838 & 1.16 & 118 & $(50,185)$ & 192 & 31 & 26.3 \\
\hline
\end{tabular}


Table C1: Primary Estimates for Each County-Set

\begin{tabular}{|c|c|c|c|c|c|c|c|c|c|}
\hline County-Set & $\begin{array}{l}\text { Expected } \\
\text { Deaths }\end{array}$ & $\begin{array}{l}\text { Expected } \\
\text { Deaths } 95 \% \text { CI }\end{array}$ & $\begin{array}{l}\text { Observed } \\
\text { Deaths }\end{array}$ & $\begin{array}{l}\text { Observed/ } \\
\text { Expected }\end{array}$ & $\begin{array}{l}\text { Excess } \\
\text { Deaths }\end{array}$ & $\begin{array}{l}\text { Excess Deaths } \\
95 \% \mathrm{CI}\end{array}$ & $\begin{array}{c}\text { Excess Deaths } \\
\text { Per } 100,000\end{array}$ & $\begin{array}{l}\text { COVID-19 } \\
\text { Deaths }\end{array}$ & $\begin{array}{l}\text { COVID-19 to } \\
\text { Excess Ratio (\%) }\end{array}$ \\
\hline Logan-Simpson-Todd, KY & 740 & $(676,802)$ & 771 & 1.04 & 31 & $(-31,95)$ & 53 & 83 & 267.7 \\
\hline $\begin{array}{l}\text { Lyon and Nearby Counties, } \\
\text { KY }\end{array}$ & 740 & $(673,810)$ & 859 & 1.16 & 119 & $(49,186)$ & 221 & 75 & 63.0 \\
\hline McCracken, KY & 813 & $(741,885)$ & 917 & 1.13 & 104 & $(32,176)$ & 160 & 82 & 78.8 \\
\hline $\begin{array}{l}\text { Marion-Nelson- } \\
\text { Washington, KY }\end{array}$ & 878 & $(810,943)$ & 944 & 1.08 & 66 & $(1,134)$ & 84 & 95 & 143.9 \\
\hline Oldham, KY & 478 & $(429,528)$ & 498 & 1.04 & 20 & $(-30,69)$ & 29 & 60 & 300.0 \\
\hline $\begin{array}{l}\text { Owen and Nearby Coun- } \\
\text { ties, KY }\end{array}$ & 784 & $(720,851)$ & 822 & 1.05 & 38 & $(-29,102)$ & 60 & 65 & 171.1 \\
\hline Pike, KY & 837 & $(767,903)$ & 969 & 1.16 & 132 & $(66,202)$ & 230 & 49 & 37.1 \\
\hline Pulaski, KY & 886 & $(818,964)$ & 969 & 1.09 & 83 & $(5,151)$ & 127 & 75 & 90.4 \\
\hline Scott-Woodford, KY & 764 & $(699,831)$ & 768 & 1.01 & 4 & $(-63,69)$ & 4 & 30 & 750.0 \\
\hline Southwest Kentucky, KY & 834 & $(765,905)$ & 959 & 1.15 & 125 & $(54,194)$ & 208 & 115 & 92.0 \\
\hline $\begin{array}{l}\text { Wolfe and Nearby Coun- } \\
\text { ties, KY }\end{array}$ & 891 & $(812,965)$ & 935 & 1.05 & 44 & $(-30,123)$ & 75 & 42 & 95.5 \\
\hline $\begin{array}{l}\text { Acadia -Jefferson Davis, } \\
\text { LA }\end{array}$ & 1067 & $(986,1147)$ & 1349 & 1.26 & 282 & $(202,363)$ & 302 & 195 & 69.1 \\
\hline Allen-Evangeline, LA & 678 & $(616,740)$ & 871 & 1.28 & 193 & $(131,255)$ & 329 & 114 & 59.1 \\
\hline Ascension, LA & 797 & $(730,863)$ & 997 & 1.25 & 200 & $(134,267)$ & 156 & 125 & 62.5 \\
\hline $\begin{array}{l}\text { Assumption-St.James- } \\
\text { St.John the Baptist, LA }\end{array}$ & 887 & $(818,954)$ & 1021 & 1.15 & 134 & $(67,203)$ & 158 & 171 & 127.6 \\
\hline $\begin{array}{l}\text { Avoyelles-East Feliciana- } \\
\text { Pointe Coupee-West } \\
\text { Feliciana, LA }\end{array}$ & 1177 & $(1093,1259)$ & 1418 & 1.20 & 241 & $(159,325)$ & 252 & 225 & 93.4 \\
\hline Beauregard -Vernon, LA & 872 & $(807,939)$ & 1026 & 1.18 & 154 & $(87,219)$ & 184 & 127 & 82.5 \\
\hline $\begin{array}{l}\text { Bienville-Natchitoches- } \\
\text { Red River, LA }\end{array}$ & 709 & $(651,765)$ & 924 & 1.30 & 215 & $(159,273)$ & 364 & 139 & 64.7 \\
\hline Bossier, LA & 1151 & $(1074,1233)$ & 1325 & 1.15 & 174 & $(92,251)$ & 137 & 191 & 109.8 \\
\hline Caddo, LA & 2788 & $(2648,2912)$ & 3560 & 1.28 & 772 & $(648,912)$ & 326 & 501 & 64.9 \\
\hline Calcasieu, LA & 2163 & $(2053,2285)$ & 2615 & 1.21 & 452 & $(330,562)$ & 221 & 273 & 60.4 \\
\hline Cameron -Vermilion, LA & 655 & $(593,717)$ & 794 & 1.21 & 139 & $(77,201)$ & 209 & 79 & 56.8 \\
\hline $\begin{array}{l}\text { Catahoula and Nearby } \\
\text { Parishes, LA }\end{array}$ & 732 & $(670,795)$ & 911 & 1.24 & 179 & $(116,241)$ & 312 & 125 & 69.8 \\
\hline Claiborne-Webster, LA & 747 & $(678,813)$ & 911 & 1.22 & 164 & $(98,233)$ & 306 & 127 & 77.4 \\
\hline De Soto-Sabine, LA & 608 & $(548,670)$ & 737 & 1.21 & 129 & $(67,189)$ & 249 & 103 & 79.8 \\
\hline East Baton Rouge, LA & 4010 & $(3865,4176)$ & 4867 & 1.21 & 857 & $(691,1002)$ & 197 & 537 & 62.7 \\
\hline Grant-Jackson-Winn, LA & 625 & $(566,686)$ & 750 & 1.20 & 125 & $(64,184)$ & 237 & 95 & 76.0 \\
\hline Iberia, LA & 764 & $(701,831)$ & 932 & 1.22 & 168 & $(101,231)$ & 244 & 90 & 53.6 \\
\hline $\begin{array}{l}\text { Iberville-West } \\
\text { Rouge, LA }\end{array}$ & 576 & $(519,633)$ & 754 & 1.31 & 178 & $(121,235)$ & 301 & 117 & 65.7 \\
\hline Jefferson, LA & 4348 & $(4174,4531)$ & 5236 & 1.20 & 888 & $(705,1062)$ & 207 & 647 & 72.9 \\
\hline Lafayette, LA & 1855 & $(1746,1955)$ & 2274 & 1.23 & 419 & $(319,528)$ & 171 & 176 & 42.0 \\
\hline Lafourche, LA & 941 & $(871,1013)$ & 1102 & 1.17 & 161 & $(89,231)$ & 164 & 152 & 94.4 \\
\hline Lincoln-Union, LA & 707 & $(648,766)$ & 920 & 1.30 & 213 & $(154,272)$ & 309 & 144 & 67.6 \\
\hline Livingston, LA & 1166 & $(1085,1249)$ & 1334 & 1.14 & 168 & $(85,249)$ & 117 & 121 & 72.0 \\
\hline Northeast Louisiana, LA & 1254 & $(1170,1344)$ & 1534 & 1.22 & 280 & $(190,364)$ & 300 & 229 & 81.8 \\
\hline Orleans, LA & 3421 & $(3276,3568)$ & 4367 & 1.28 & 946 & $(799,1091)$ & 243 & 578 & 61.1 \\
\hline Ouachita, LA & 1650 & $(1555,1757)$ & 2014 & 1.22 & 364 & $(257,459)$ & 239 & 303 & 83.2 \\
\hline $\begin{array}{l}\text { Plaquemines-St. Bernard, } \\
\text { LA }\end{array}$ & 586 & $(529,643)$ & 683 & 1.17 & 97 & $(40,154)$ & 135 & 65 & 67.0 \\
\hline Rapides, LA & 1558 & $(1461,1663)$ & 1887 & 1.21 & 329 & $(224,426)$ & 255 & 223 & 67.8 \\
\hline St. Charles, LA & 455 & $(408,504)$ & 537 & 1.18 & 82 & $(33,129)$ & 155 & 64 & 78.0 \\
\hline $\begin{array}{l}\text { St. Helena -Tangipahoa - } \\
\text { Washington, LA }\end{array}$ & 2128 & $(2009,2244)$ & 2423 & 1.14 & 295 & $(179,414)$ & 153 & 262 & 88.8 \\
\hline St. Landry, LA & 1062 & $(980,1145)$ & 1257 & 1.18 & 195 & $(112,277)$ & 239 & 192 & 98.5 \\
\hline St. Martin, LA & 534 & $(478,584)$ & 677 & 1.27 & 143 & $(93,199)$ & 271 & 93 & 65.0 \\
\hline
\end{tabular}


Table C1: Primary Estimates for Each County-Set

\begin{tabular}{|c|c|c|c|c|c|c|c|c|c|}
\hline County-Set & $\begin{array}{l}\text { Expected } \\
\text { Deaths }\end{array}$ & $\begin{array}{l}\text { Expected } \\
\text { Deaths } 95 \% \text { CI }\end{array}$ & $\begin{array}{l}\text { Observed } \\
\text { Deaths }\end{array}$ & $\begin{array}{l}\text { Observed/ } \\
\text { Expected }\end{array}$ & $\begin{array}{l}\text { Excess } \\
\text { Deaths }\end{array}$ & $\begin{array}{l}\text { Excess Deaths } \\
95 \% \text { CI }\end{array}$ & $\begin{array}{l}\text { Excess Deaths } \\
\text { Per } 100,000\end{array}$ & $\begin{array}{l}\text { COVID-19 } \\
\text { Deaths }\end{array}$ & $\begin{array}{c}\text { COVID-19 to } \\
\text { Excess Ratio (\%) }\end{array}$ \\
\hline St. Mary, LA & 565 & $(510,620)$ & 689 & 1.22 & 124 & $(69,179)$ & 258 & 95 & 76.6 \\
\hline St. Tammany, LA & 2423 & $(2294,2544)$ & 2787 & 1.15 & 364 & $(243,493)$ & 139 & 275 & 75.5 \\
\hline Terrebonne, LA & 1075 & $(994,1157)$ & 1258 & 1.17 & 183 & $(101,264)$ & 167 & 128 & 69.9 \\
\hline Androscoggin, $\mathrm{ME}$ & 1273 & $(1184,1363)$ & 1264 & 0.99 & -9 & $(-99,80)$ & -9 & 45 & NA \\
\hline Aroostook, ME & 890 & $(818,972)$ & 963 & 1.08 & 73 & $(-9,145)$ & 110 & 24 & 32.9 \\
\hline Cumberland, ME & 2871 & $(2730,3019)$ & 2960 & 1.03 & 89 & $(-59,230)$ & 30 & 112 & 125.8 \\
\hline Franklin-Oxford, ME & 1108 & $(1024,1184)$ & 1062 & 0.96 & -46 & $(-122,38)$ & -52 & 44 & NA \\
\hline Hancock-Washington, ME & 1123 & $(1045,1208)$ & 1212 & 1.08 & 89 & $(4,167)$ & 103 & 32 & 36.0 \\
\hline Kennebec, ME & 1406 & $(1316,1496)$ & 1459 & 1.04 & 53 & $(-37,143)$ & 43 & 36 & 67.9 \\
\hline Knox-Waldo, ME & 992 & $(915,1062)$ & 943 & 0.95 & -49 & $(-119,28)$ & -62 & 27 & NA \\
\hline Lincoln-Sagadahoc, ME & 824 & $(757,896)$ & 843 & 1.02 & 19 & $(-53,86)$ & 27 & 10 & 52.6 \\
\hline $\begin{array}{l}\text { Penobscot-Piscataquis, } \\
\text { ME }\end{array}$ & 2048 & $(1931,2165)$ & 2090 & 1.02 & 42 & $(-75,159)$ & 25 & 42 & 100.0 \\
\hline Somerset, ME & 673 & $(604,733)$ & 663 & 0.99 & -10 & $(-70,59)$ & -19 & 20 & NA \\
\hline York, ME & 2308 & $(2188,2422)$ & 2282 & 0.99 & -26 & $(-140,94)$ & -13 & 93 & NA \\
\hline Allegany-Garrett, MD & 1286 & $(1200,1380)$ & 1606 & 1.25 & 320 & $(226,406)$ & 323 & 231 & 72.2 \\
\hline Anne Arundel, MD & 4708 & $(4526,4903)$ & 5026 & 1.07 & 318 & $(123,500)$ & 54 & 398 & 125.2 \\
\hline Baltimore (city), MD & 6646 & $(6411,6890)$ & 7797 & 1.17 & 1151 & $(907,1386)$ & 196 & 783 & 68.0 \\
\hline Baltimore (county), MD & 8811 & $(8535,9086)$ & 9901 & 1.12 & 1090 & $(815,1366)$ & 132 & 1024 & 93.9 \\
\hline Calvert, MD & 756 & $(693,826)$ & 825 & 1.09 & 69 & $(-1,132)$ & 74 & 55 & 79.7 \\
\hline $\begin{array}{l}\text { Caroline-Dorchester- } \\
\text { Talbot, MD }\end{array}$ & 1272 & $(1185,1362)$ & 1406 & 1.11 & 134 & $(44,221)$ & 131 & 60 & 44.8 \\
\hline Carroll, MD & 1761 & $(1659,1865)$ & 1846 & 1.05 & 85 & $(-19,187)$ & 50 & 196 & 230.6 \\
\hline Cecil, MD & 1095 & $(1022,1172)$ & 1219 & 1.11 & 124 & $(47,197)$ & 120 & 100 & 80.6 \\
\hline Charles, MD & 1225 & $(1145,1308)$ & 1435 & 1.17 & 210 & $(127,290)$ & 127 & 165 & 78.6 \\
\hline Frederick, MD & 2071 & $(1961,2182)$ & 2214 & 1.07 & 143 & $(32,253)$ & 54 & 207 & 144.8 \\
\hline Harford, MD & 2375 & $(2257,2487)$ & 2581 & 1.09 & 206 & $(94,324)$ & 80 & 178 & 86.4 \\
\hline Howard, MD & 1878 & $(1776,1987)$ & 2079 & 1.11 & 201 & $(92,303)$ & 61 & 210 & 104.5 \\
\hline Kent-Queen Anne's, MD & 758 & $(692,827)$ & 784 & 1.03 & 26 & $(-43,92)$ & 37 & 66 & 253.8 \\
\hline Montgomery, MD & 6640 & $(6428,6847)$ & 7709 & 1.16 & 1069 & $(862,1281)$ & 101 & 1213 & 113.5 \\
\hline Prince George's, MD & 6446 & $(6242,6654)$ & 8374 & 1.30 & 1928 & $(1720,2132)$ & 212 & 1355 & 70.3 \\
\hline St. Mary's, MD & 902 & $(833,971)$ & 1008 & 1.12 & 106 & $(37,175)$ & 93 & 97 & 91.5 \\
\hline Somerset-Worcester, MD & 958 & $(885,1029)$ & 1093 & 1.14 & 135 & $(64,208)$ & 175 & 78 & 57.8 \\
\hline Washington, MD & 1730 & $(1620,1836)$ & 1969 & 1.14 & 239 & $(133,349)$ & 157 & 200 & 83.7 \\
\hline Wicomico, MD & 1092 & $(1014,1174)$ & 1201 & 1.10 & 109 & $(27,187)$ & 104 & 90 & 82.6 \\
\hline $\begin{array}{l}\text { Barnstable-Dukes- } \\
\text { Nantucket, MA }\end{array}$ & 3343 & $(3195,3504)$ & 3383 & 1.01 & 40 & $(-121,188)$ & 17 & 223 & 557.5 \\
\hline Berkshire, MA & 1648 & $(1550,1754)$ & 1676 & 1.02 & 28 & $(-78,126)$ & 22 & 127 & 453.6 \\
\hline Bristol, MA & 5755 & $(5541,5972)$ & 6433 & 1.12 & 678 & $(461,892)$ & 119 & 868 & 128.0 \\
\hline Essex, MA & 7421 & $(7170,7659)$ & 8356 & 1.13 & 935 & $(697,1186)$ & 117 & 1354 & 144.8 \\
\hline Franklin, MA & 759 & $(694,828)$ & 839 & 1.11 & 80 & $(11,145)$ & 112 & 78 & 97.5 \\
\hline Hampden, MA & 4871 & $(4686,5063)$ & 5684 & 1.17 & 813 & $(621,998)$ & 172 & 832 & 102.3 \\
\hline Hampshire, MA & 1362 & $(1275,1453)$ & 1493 & 1.10 & 131 & $(40,218)$ & 81 & 167 & 127.5 \\
\hline Middlesex, MA & 11967 & $(11642,12294)$ & 13664 & 1.14 & 1697 & $(1370,2022)$ & 104 & 2274 & 134.0 \\
\hline Norfolk, MA & 6102 & $(5873,6300)$ & 6816 & 1.12 & 714 & $(516,943)$ & 100 & 1000 & 140.1 \\
\hline Plymouth, MA & 5072 & $(4886,5269)$ & 5685 & 1.12 & 613 & $(416,799)$ & 117 & 787 & 128.4 \\
\hline Suffolk, MA & 4832 & $(4654,5011)$ & 5924 & 1.23 & 1092 & $(913,1270)$ & 134 & 1172 & 107.3 \\
\hline Worcester, MA & 7741 & $(7483,7999)$ & 8260 & 1.07 & 519 & $(261,777)$ & 62 & 1221 & 235.3 \\
\hline $\begin{array}{l}\text { Alcona-Alpena-Presque } \\
\text { Isle, MI }\end{array}$ & 796 & $(726,874)$ & 819 & 1.03 & 23 & $(-55,93)$ & 46 & 59 & 256.5 \\
\hline $\begin{array}{l}\text { Alger-Delta-Schoolcraft, } \\
\text { MI }\end{array}$ & 724 & $(662,788)$ & 825 & 1.14 & 101 & $(37,163)$ & 191 & 78 & 77.2 \\
\hline Allegan, MI & 1094 & $(1011,1170)$ & 1259 & 1.15 & 165 & $(89,248)$ & 139 & 81 & 49.1 \\
\hline $\begin{array}{l}\text { Antrim-Charlevoix- } \\
\text { Kalkaska-Otsego, MI }\end{array}$ & 1108 & $(1030,1191)$ & 1165 & 1.05 & 57 & $(-26,135)$ & 61 & 58 & 101.8 \\
\hline Arenac-Iosco-Ogemaw, MI & 1006 & $(927,1086)$ & 1062 & 1.06 & 56 & $(-24,135)$ & 91 & 92 & 164.3 \\
\hline
\end{tabular}


Table C1: Primary Estimates for Each County-Set

\begin{tabular}{|c|c|c|c|c|c|c|c|c|c|}
\hline County-Set & $\begin{array}{l}\text { Expected } \\
\text { Deaths }\end{array}$ & $\begin{array}{l}\text { Expected } \\
\text { Deaths } 95 \% \text { CI }\end{array}$ & $\begin{array}{l}\text { Observed } \\
\text { Deaths }\end{array}$ & $\begin{array}{l}\text { Observed/ } \\
\text { Expected }\end{array}$ & $\begin{array}{l}\text { Excess } \\
\text { Deaths }\end{array}$ & $\begin{array}{l}\text { Excess Deaths } \\
95 \% \mathrm{CI}\end{array}$ & $\begin{array}{l}\text { Excess Deaths } \\
\text { Per } 100,000\end{array}$ & $\begin{array}{l}\text { COVID-19 } \\
\text { Deaths }\end{array}$ & $\begin{array}{l}\text { COVID-19 to } \\
\text { Excess Ratio (\%) }\end{array}$ \\
\hline Barry, MI & 605 & $(547,667)$ & 634 & 1.05 & 29 & $(-33,87)$ & 47 & 42 & 144.8 \\
\hline Bay, MI & 1357 & $(1260,1450)$ & 1536 & 1.13 & 179 & $(86,276)$ & 173 & 173 & 96.6 \\
\hline $\begin{array}{l}\text { Benzie-Manistee-Mason, } \\
\text { MI }\end{array}$ & 895 & $(829,965)$ & 1076 & 1.20 & 181 & $(111,247)$ & 251 & 71 & 39.2 \\
\hline Berrien, MI & 1842 & $(1737,1947)$ & 1918 & 1.04 & 76 & $(-29,181)$ & 50 & 195 & 256.6 \\
\hline Branch-Hillsdale, MI & 1035 & $(956,1117)$ & 1183 & 1.14 & 148 & $(66,227)$ & 165 & 146 & 98.6 \\
\hline Calhoun, MI & 1641 & $(1538,1743)$ & 1919 & 1.17 & 278 & $(176,381)$ & 207 & 178 & 64.0 \\
\hline Cass, MI & 565 & $(511,620)$ & 669 & 1.18 & 104 & $(49,158)$ & 200 & 68 & 65.4 \\
\hline Cheboygan-Emmet, MI & 755 & $(685,824)$ & 833 & 1.10 & 78 & $(9,148)$ & 133 & 75 & 96.2 \\
\hline $\begin{array}{l}\text { Chippewa-Luce-Mackinac, } \\
\text { MI }\end{array}$ & 637 & $(577,699)$ & 612 & 0.96 & -25 & $(-87,35)$ & -47 & 27 & NA \\
\hline Clare-Gladwin, MI & 896 & $(821,966)$ & 960 & 1.07 & 64 & $(-6,139)$ & 114 & 83 & 129.7 \\
\hline Clinton, MI & 659 & $(591,723)$ & 739 & 1.12 & 80 & $(16,148)$ & 99 & 52 & 65.0 \\
\hline $\begin{array}{l}\text { Crawford-Montmorency- } \\
\text { Oscoda-Roscommon, MI }\end{array}$ & 938 & $(863,1014)$ & 1014 & 1.08 & 76 & $(0,151)$ & 137 & 69 & 90.8 \\
\hline $\begin{array}{l}\text { Dickinson-Iron- } \\
\text { Menominee, MI }\end{array}$ & 811 & $(742,881)$ & 960 & 1.18 & 149 & $(79,218)$ & 251 & 127 & 85.2 \\
\hline Eaton, MI & 1108 & $(1029,1181)$ & 1241 & 1.12 & 133 & $(60,212)$ & 120 & 83 & 62.4 \\
\hline Genesee, MI & 4968 & $(4759,5162)$ & 5525 & 1.11 & 557 & $(363,766)$ & 138 & 528 & 94.8 \\
\hline $\begin{array}{l}\text { Grand Traverse-Leelanau, } \\
\text { MI }\end{array}$ & 1228 & $(1144,1301)$ & 1337 & 1.09 & 109 & $(36,193)$ & 94 & 56 & 51.4 \\
\hline Gratiot-Isabella, MI & 1023 & $(944,1101)$ & 1189 & 1.16 & 166 & $(88,245)$ & 151 & 91 & 54.8 \\
\hline Huron-Sanilac, MI & 1030 & $(948,1103)$ & 1069 & 1.04 & 39 & $(-34,121)$ & 54 & 121 & 310.3 \\
\hline Ingham, MI & 2333 & $(2216,2447)$ & 2669 & 1.14 & 336 & $(222,453)$ & 114 & 175 & 52.1 \\
\hline Ionia, MI & 578 & $(522,638)$ & 610 & 1.06 & 32 & $(-28,88)$ & 49 & 40 & 125.0 \\
\hline Jackson, MI & 1860 & $(1748,1970)$ & 2005 & 1.08 & 145 & $(35,257)$ & 91 & 198 & 136.6 \\
\hline Kalamazoo, MI & 2302 & $(2185,2419)$ & 2558 & 1.11 & 256 & $(139,373)$ & 96 & 212 & 82.8 \\
\hline Kent, MI & 5103 & $(4912,5290)$ & 5706 & 1.12 & 603 & $(416,794)$ & 91 & 511 & 84.7 \\
\hline $\begin{array}{l}\text { Lake-Missaukee-Wexford, } \\
\text { MI }\end{array}$ & 731 & $(669,800)$ & 792 & 1.08 & 61 & $(-8,123)$ & 99 & 28 & 45.9 \\
\hline Lapeer, MI & 984 & $(909,1064)$ & 1037 & 1.05 & 53 & $(-27,128)$ & 60 & 101 & 190.6 \\
\hline Lenawee, MI & 1126 & $(1043,1208)$ & 1241 & 1.10 & 115 & $(33,198)$ & 117 & 83 & 72.2 \\
\hline Livingston, MI & 1560 & $(1465,1656)$ & 1774 & 1.14 & 214 & $(118,309)$ & 111 & 90 & 42.1 \\
\hline Macomb, MI & 9194 & $(8903,9483)$ & 10877 & 1.18 & 1683 & $(1394,1974)$ & 191 & 1491 & 88.6 \\
\hline Marquette, MI & 734 & $(671,802)$ & 723 & 0.99 & -11 & $(-79,52)$ & -17 & 47 & NA \\
\hline Mecosta-Osceola, MI & 691 & $(627,754)$ & 775 & 1.12 & 84 & $(21,148)$ & 125 & 37 & 44.0 \\
\hline Midland, MI & 789 & $(718,861)$ & 911 & 1.15 & 122 & $(50,193)$ & 147 & 48 & 39.3 \\
\hline Monroe, MI & 1625 & $(1531,1717)$ & 1793 & 1.10 & 168 & $(76,262)$ & 110 & 141 & 83.9 \\
\hline Montcalm, MI & 684 & $(625,747)$ & 734 & 1.07 & 50 & $(-13,109)$ & 78 & 79 & 158.0 \\
\hline Muskegon, MI & 1836 & $(1733,1935)$ & 2196 & 1.20 & 360 & $(261,463)$ & 206 & 279 & 77.5 \\
\hline Newaygo-Oceana, MI & 836 & $(766,909)$ & 910 & 1.09 & 74 & $(1,144)$ & 96 & 78 & 105.4 \\
\hline $\begin{array}{l}\text { Northwest Upper Penin- } \\
\text { sula, MI }\end{array}$ & 800 & $(731,867)$ & 943 & 1.18 & 143 & $(76,212)$ & 215 & 130 & 90.9 \\
\hline Oakland, MI & 11028 & $(10713,11346)$ & 12930 & 1.17 & 1902 & $(1584,2217)$ & 150 & 1539 & 80.9 \\
\hline Ottawa, MI & 2186 & $(2072,2306)$ & 2509 & 1.15 & 323 & $(203,437)$ & 108 & 248 & 76.8 \\
\hline Saginaw, MI & 2260 & $(2140,2381)$ & 2707 & 1.20 & 447 & $(326,567)$ & 237 & 372 & 83.2 \\
\hline St. Clair, MI & 1899 & $(1790,2017)$ & 2104 & 1.11 & 205 & $(87,314)$ & 129 & 172 & 83.9 \\
\hline St. Joseph, MI & 714 & $(651,779)$ & 759 & 1.06 & 45 & $(-20,108)$ & 74 & 64 & 142.2 \\
\hline Shiawassee, MI & 791 & $(727,863)$ & 847 & 1.07 & 56 & $(-16,120)$ & 82 & 58 & 103.6 \\
\hline Tuscola, MI & 676 & $(612,738)$ & 775 & 1.15 & 99 & $(37,163)$ & 191 & 100 & 101.0 \\
\hline Van Buren, MI & 826 & $(755,891)$ & 914 & 1.11 & 88 & $(23,159)$ & 116 & 93 & 105.7 \\
\hline Washtenaw, MI & 2462 & $(2342,2585)$ & 2709 & 1.10 & 247 & $(124,367)$ & 66 & 208 & 84.2 \\
\hline Wayne, MI & 18721 & $(18198,19216)$ & 23033 & 1.23 & 4312 & $(3817,4835)$ & 247 & 3281 & 76.1 \\
\hline Aitkin-Itasca, MN & 767 & $(703,830)$ & 829 & 1.08 & 62 & $(-1,126)$ & 101 & 76 & 122.6 \\
\hline Anoka, MN & 2433 & $(2311,2551)$ & 2754 & 1.13 & 321 & $(203,443)$ & 89 & 380 & 118.4 \\
\hline
\end{tabular}


Table C1: Primary Estimates for Each County-Set

\begin{tabular}{|c|c|c|c|c|c|c|c|c|c|}
\hline County-Set & $\begin{array}{l}\text { Expected } \\
\text { Deaths }\end{array}$ & $\begin{array}{l}\text { Expected } \\
\text { Deaths } 95 \% \mathrm{CI}\end{array}$ & $\begin{array}{l}\text { Observed } \\
\text { Deaths }\end{array}$ & $\begin{array}{l}\text { Observed/ } \\
\text { Expected }\end{array}$ & $\begin{array}{l}\text { Excess } \\
\text { Deaths }\end{array}$ & $\begin{array}{l}\text { Excess Deaths } \\
95 \% \mathrm{CI}\end{array}$ & $\begin{array}{l}\text { Excess Deaths } \\
\text { Per } 100,000\end{array}$ & $\begin{array}{l}\text { COVID-19 } \\
\text { Deaths }\end{array}$ & $\begin{array}{l}\text { COVID-19 to } \\
\text { Excess Ratio (\%) }\end{array}$ \\
\hline $\begin{array}{l}\text { Becker-Hubbard- } \\
\text { Mahnomen-Norman, } \\
\text { MN }\end{array}$ & 807 & $(737,885)$ & 863 & 1.07 & 56 & $(-22,126)$ & 82 & 100 & 178.6 \\
\hline Beltrami-Clearwater, MN & 516 & $(464,572)$ & 662 & 1.28 & 146 & $(90,198)$ & 260 & 60 & 41.1 \\
\hline Benton-Stearns, MN & 1543 & $(1448,1638)$ & 1864 & 1.21 & 321 & $(226,416)$ & 158 & 279 & 86.9 \\
\hline $\begin{array}{l}\text { Blue Earth-Faribault- } \\
\text { Martin-Watonwan, MN }\end{array}$ & 1086 & $(1009,1161)$ & 1145 & 1.05 & 59 & $(-16,136)$ & 52 & 86 & 145.8 \\
\hline $\begin{array}{l}\text { Brown-Le Sueur-Nicollet, } \\
\text { MN }\end{array}$ & 837 & $(772,906)$ & 847 & 1.01 & 10 & $(-59,75)$ & 11 & 85 & 850.0 \\
\hline Carver, MN & 583 & $(531,641)$ & 616 & 1.06 & 33 & $(-25,85)$ & 31 & 39 & 118.2 \\
\hline Cass-Crow Wing, MN & 1106 & $(1024,1187)$ & 1137 & 1.03 & 31 & $(-50,113)$ & 33 & 103 & 332.3 \\
\hline Chisago-Isanti, MN & 853 & $(783,923)$ & 872 & 1.02 & 19 & $(-51,89)$ & 19 & 70 & 368.4 \\
\hline Clay, MN & 500 & $(449,555)$ & 568 & 1.14 & 68 & $(13,119)$ & 105 & 91 & 133.8 \\
\hline Dakota, MN & 2855 & $(2727,2979)$ & 3210 & 1.12 & 355 & $(231,483)$ & 82 & 318 & 89.6 \\
\hline Dodge-Steele-Waseca, MN & 700 & $(639,765)$ & 717 & 1.02 & 17 & $(-48,78)$ & 22 & 34 & 200.0 \\
\hline $\begin{array}{l}\text { Douglas-Morrison-Todd, } \\
\text { MN }\end{array}$ & 1037 & $(957,1114)$ & 1150 & 1.11 & 113 & $(36,193)$ & 117 & 142 & 125.7 \\
\hline $\begin{array}{l}\text { Fillmore-Houston-Winona, } \\
\text { MN }\end{array}$ & 903 & $(825,970)$ & 947 & 1.05 & 44 & $(-23,122)$ & 48 & 73 & 165.9 \\
\hline Freeborn-Mower, MN & 818 & $(751,892)$ & 821 & 1.00 & 3 & $(-71,70)$ & 5 & 53 & 1766.7 \\
\hline Goodhue-Wabasha, MN & 708 & $(648,772)$ & 806 & 1.14 & 98 & $(34,158)$ & 144 & 55 & 56.1 \\
\hline Hennepin, MN & 9202 & $(8928,9479)$ & 10686 & 1.16 & 1484 & $(1207,1758)$ & 116 & 1477 & 99.5 \\
\hline $\begin{array}{l}\text { Kanabec-Mille Lacs-Pine, } \\
\text { MN }\end{array}$ & 810 & $(741,881)$ & 932 & 1.15 & 122 & $(51,191)$ & 167 & 93 & 76.2 \\
\hline Kandiyohi-Renville, MN & 574 & $(521,629)$ & 667 & 1.16 & 93 & $(38,146)$ & 162 & 108 & 116.1 \\
\hline $\begin{array}{l}\text { McLeod-Meeker-Sibley, } \\
\text { MN }\end{array}$ & 749 & $(686,811)$ & 839 & 1.12 & 90 & $(28,153)$ & 122 & 82 & 91.1 \\
\hline Northwest Minnesota, MN & 834 & $(762,902)$ & 1036 & 1.24 & 202 & $(134,274)$ & 247 & 143 & 70.8 \\
\hline Olmsted, MN & 1186 & $(1105,1268)$ & 1273 & 1.07 & 87 & $(5,168)$ & 55 & 71 & 81.6 \\
\hline $\begin{array}{l}\text { Otter Tail-Wadena-Wilkin, } \\
\text { MN }\end{array}$ & 1002 & $(931,1083)$ & 1087 & 1.08 & 85 & $(4,156)$ & 106 & 83 & 97.6 \\
\hline Ramsey, MN & 4508 & $(4338,4686)$ & 5141 & 1.14 & 633 & $(455,803)$ & 113 & 713 & 112.6 \\
\hline Rice, MN & 548 & $(497,602)$ & 593 & 1.08 & 45 & $(-9,96)$ & 67 & 57 & 126.7 \\
\hline $\begin{array}{l}\text { St. Louis and Nearby } \\
\text { Counties, MN }\end{array}$ & 2856 & $(2726,3002)$ & 3296 & 1.15 & 440 & $(294,570)$ & 167 & 316 & 71.8 \\
\hline Scott, MN & 768 & $(698,831)$ & 878 & 1.14 & 110 & $(47,180)$ & 73 & 77 & 70.0 \\
\hline Sherburne, MN & 606 & $(549,663)$ & 705 & 1.16 & 99 & $(42,156)$ & 100 & 63 & 63.6 \\
\hline Southwest Minnesota, MN & 777 & $(711,843)$ & 872 & 1.12 & 95 & $(29,161)$ & 136 & 118 & 124.2 \\
\hline $\begin{array}{l}\text { Stevens and Nearby Coun- } \\
\text { ties, MN }\end{array}$ & 714 & $(653,781)$ & 761 & 1.07 & 47 & $(-20,108)$ & 82 & 75 & 159.6 \\
\hline Washington, MN & 1875 & $(1778,1976)$ & 2030 & 1.08 & 155 & $(54,252)$ & 58 & 206 & 132.9 \\
\hline Wright, MN & 932 & $(858,1008)$ & 992 & 1.06 & 60 & $(-16,134)$ & 43 & 104 & 173.3 \\
\hline $\begin{array}{l}\text { Yellow Medicine and } \\
\text { Nearby Counties, MN }\end{array}$ & 677 & $(618,740)$ & 774 & 1.14 & 97 & $(34,156)$ & 155 & 102 & 105.2 \\
\hline $\begin{array}{l}\text { Adams-Amite-Wilkinson, } \\
\text { MS }\end{array}$ & 739 & $(676,810)$ & 832 & 1.13 & 93 & $(22,156)$ & 180 & 106 & 114.0 \\
\hline $\begin{array}{l}\text { Alcorn-Prentiss- } \\
\text { Tishomingo, MS }\end{array}$ & 1124 & $(1041,1204)$ & 1306 & 1.16 & 182 & $(102,265)$ & 224 & 144 & 79.1 \\
\hline $\begin{array}{l}\text { Attala-Choctaw-Leake- } \\
\text { Webster, MS }\end{array}$ & 789 & $(717,858)$ & 928 & 1.18 & 139 & $(70,211)$ & 237 & 142 & 102.2 \\
\hline $\begin{array}{l}\text { Benton-Marshall-Tippah, } \\
\text { MS }\end{array}$ & 842 & $(770,922)$ & 978 & 1.16 & 136 & $(56,208)$ & 208 & 125 & 91.9 \\
\hline Bolivar-Sunflower, MS & 718 & $(657,790)$ & 989 & 1.38 & 271 & $(199,332)$ & 490 & 156 & 57.6 \\
\hline $\begin{array}{l}\text { Calhoun-Chickasaw- } \\
\text { Grenada, MS }\end{array}$ & 714 & $(650,782)$ & 877 & 1.23 & 163 & $(95,227)$ & 310 & 114 & 69.9 \\
\hline
\end{tabular}


Table C1: Primary Estimates for Each County-Set

\begin{tabular}{|c|c|c|c|c|c|c|c|c|c|}
\hline County-Set & $\begin{array}{l}\text { Expected } \\
\text { Deaths }\end{array}$ & $\begin{array}{l}\text { Expected } \\
\text { Deaths } 95 \% \text { CI }\end{array}$ & $\begin{array}{l}\text { Observed } \\
\text { Deaths }\end{array}$ & $\begin{array}{l}\text { Observed/ } \\
\text { Expected }\end{array}$ & $\begin{array}{l}\text { Excess } \\
\text { Deaths }\end{array}$ & $\begin{array}{l}\text { Excess Deaths } \\
95 \% \mathrm{CI}\end{array}$ & $\begin{array}{l}\text { Excess Deaths } \\
\text { Per } 100,000\end{array}$ & $\begin{array}{l}\text { COVID-19 } \\
\text { Deaths }\end{array}$ & $\begin{array}{l}\text { COVID-19 to } \\
\text { Excess Ratio (\%) }\end{array}$ \\
\hline $\begin{array}{l}\text { Carroll-Leflore- } \\
\text { Montgomery, MS }\end{array}$ & 627 & $(565,683)$ & 919 & 1.47 & 292 & $(236,354)$ & 615 & 169 & 57.9 \\
\hline $\begin{array}{l}\text { Claiborne-Jefferson- } \\
\text { Warren, MS }\end{array}$ & 743 & $(678,809)$ & 902 & 1.21 & 159 & $(93,224)$ & 261 & 126 & 79.2 \\
\hline Clarke-Jasper-Wayne, MS & 650 & $(590,713)$ & 763 & 1.17 & 113 & $(50,173)$ & 220 & 114 & 100.9 \\
\hline Clay-Oktibbeha, MS & 578 & $(520,633)$ & 729 & 1.26 & 151 & $(96,209)$ & 220 & 113 & 74.8 \\
\hline $\begin{array}{l}\text { Coahoma-Quitman- } \\
\text { Tallahatchie-Tunica, } \\
\text { MS }\end{array}$ & 659 & $(595,719)$ & 882 & 1.34 & 223 & $(163,287)$ & 431 & 125 & 56.1 \\
\hline $\begin{array}{l}\text { Copiah-Franklin-Lincoln, } \\
\text { MS }\end{array}$ & 870 & $(797,938)$ & 1084 & 1.25 & 214 & $(146,287)$ & 304 & 156 & 72.9 \\
\hline $\begin{array}{l}\text { Covington-Simpson-Smith, } \\
\text { MS }\end{array}$ & 777 & $(710,844)$ & 956 & 1.23 & 179 & $(112,246)$ & 295 & 137 & 76.5 \\
\hline De Soto, MS & 1572 & $(1479,1670)$ & 1754 & 1.12 & 182 & $(84,275)$ & 97 & 174 & 95.6 \\
\hline Forrest, MS & 827 & $(760,896)$ & 924 & 1.12 & 97 & $(28,164)$ & 131 & 101 & 104.1 \\
\hline $\begin{array}{l}\text { George-Greene-Perry- } \\
\text { Stone, MS }\end{array}$ & 809 & $(743,880)$ & 951 & 1.18 & 142 & $(71,208)$ & 205 & 105 & 73.9 \\
\hline Hancock-Harrison, MS & 2834 & $(2691,2976)$ & 3063 & 1.08 & 229 & $(87,372)$ & 89 & 234 & 102.2 \\
\hline Hinds, MS & 2319 & $(2201,2433)$ & 2870 & 1.24 & 551 & $(437,669)$ & 238 & 284 & 51.5 \\
\hline Itawamba-Monroe, MS & 773 & $(703,841)$ & 953 & 1.23 & 180 & $(112,250)$ & 308 & 141 & 78.3 \\
\hline Jackson, MS & 1511 & $(1410,1603)$ & 1683 & 1.11 & 172 & $(80,273)$ & 119 & 169 & 98.3 \\
\hline $\begin{array}{l}\text { Jefferson Davis-Lawrence- } \\
\text { Marion, MS }\end{array}$ & 642 & $(580,705)$ & 826 & 1.29 & 184 & $(121,246)$ & 390 & 112 & 60.9 \\
\hline Jones, MS & 850 & $(785,923)$ & 900 & 1.06 & 50 & $(-23,115)$ & 72 & 98 & 196.0 \\
\hline $\begin{array}{l}\text { Kemper-Neshoba- } \\
\text { Noxubee-Winston, MS }\end{array}$ & 914 & $(841,987)$ & 1153 & 1.26 & 239 & $(166,312)$ & 359 & 234 & 97.9 \\
\hline Lafayette-Yalobusha, MS & 592 & $(532,654)$ & 737 & 1.24 & 145 & $(83,205)$ & 212 & 101 & 69.7 \\
\hline Lamar, MS & 466 & $(416,518)$ & 562 & 1.21 & 96 & $(44,146)$ & 148 & 54 & 56.2 \\
\hline Lauderdale-Newton, MS & 1139 & $(1063,1218)$ & 1507 & 1.32 & 368 & $(289,444)$ & 388 & 201 & 54.6 \\
\hline Lee, MS & 948 & $(872,1019)$ & 1136 & 1.20 & 188 & $(117,264)$ & 219 & 139 & 73.9 \\
\hline Lowndes, MS & 690 & $(629,761)$ & 802 & 1.16 & 112 & $(41,173)$ & 192 & 104 & 92.9 \\
\hline Madison, MS & 1041 & $(965,1124)$ & 1186 & 1.14 & 145 & $(62,221)$ & 134 & 136 & 93.8 \\
\hline Panola-Tate, MS & 736 & $(673,800)$ & 893 & 1.21 & 157 & $(93,220)$ & 249 & 143 & 91.1 \\
\hline Pearl River, MS & 753 & $(681,821)$ & 849 & 1.13 & 96 & $(28,168)$ & 173 & 88 & 91.7 \\
\hline Pike-Walthall, MS & 760 & $(694,831)$ & 855 & 1.12 & 95 & $(24,161)$ & 176 & 104 & 109.5 \\
\hline Pontotoc-Union, MS & 662 & $(602,726)$ & 780 & 1.18 & 118 & $(54,178)$ & 193 & 93 & 78.8 \\
\hline Rankin-Scott, MS & 1585 & $(1493,1685)$ & 1967 & 1.24 & 382 & $(282,474)$ & 208 & 214 & 56.0 \\
\hline Washington, MS & 583 & $(524,640)$ & 790 & 1.36 & 207 & $(150,266)$ & 483 & 106 & 51.2 \\
\hline $\begin{array}{l}\text { Yazoo and Nearby Coun- } \\
\text { ties, MS }\end{array}$ & 724 & $(663,791)$ & 890 & 1.23 & 166 & $(99,227)$ & 283 & 161 & 97.0 \\
\hline $\begin{array}{l}\text { Andrew-Atchison-Holt- } \\
\text { Nodaway, MO }\end{array}$ & 491 & $(435,543)$ & 586 & 1.19 & 95 & $(43,151)$ & 193 & 70 & 73.7 \\
\hline Audrain-Callaway, MO & 775 & $(710,845)$ & 908 & 1.17 & 133 & $(63,198)$ & 189 & 92 & 69.2 \\
\hline $\begin{array}{l}\text { Barry-Lawrence- } \\
\text { McDonald, MO }\end{array}$ & 1272 & $(1180,1365)$ & 1375 & 1.08 & 103 & $(10,195)$ & 104 & 143 & 138.8 \\
\hline $\begin{array}{l}\text { Benton-Henry-St. Clair, } \\
\text { MO }\end{array}$ & 787 & $(717,854)$ & 895 & 1.14 & 108 & $(41,178)$ & 212 & 77 & 71.3 \\
\hline $\begin{array}{l}\text { Bollinger-Madison- } \\
\text { Stoddard-Wayne, MO }\end{array}$ & 912 & $(843,985)$ & 1074 & 1.18 & 162 & $(89,231)$ & 247 & 111 & 68.5 \\
\hline Boone, MO & 1227 & $(1148,1305)$ & 1402 & 1.14 & 175 & $(97,254)$ & 96 & 95 & 54.3 \\
\hline Buchanan, MO & 939 & $(864,1014)$ & 1132 & 1.21 & 193 & $(118,268)$ & 219 & 125 & 64.8 \\
\hline Butler-Carter-Ripley, MO & 873 & $(800,944)$ & 999 & 1.14 & 126 & $(55,199)$ & 205 & 81 & 64.3 \\
\hline $\begin{array}{l}\text { Camden-Miller-Moniteau- } \\
\text { Morgan, MO }\end{array}$ & 1292 & $(1202,1378)$ & 1530 & 1.18 & 238 & $(152,328)$ & 218 & 184 & 77.3 \\
\hline Cape Girardeau-Perry, MO & 1061 & $(984,1136)$ & 1337 & 1.26 & 276 & $(201,353)$ & 280 & 146 & 52.9 \\
\hline Carroll-Lafayette-Ray, MO & 835 & $(766,909)$ & 938 & 1.12 & 103 & $(29,172)$ & 160 & 102 & 99.0 \\
\hline
\end{tabular}


Table C1: Primary Estimates for Each County-Set

\begin{tabular}{|c|c|c|c|c|c|c|c|c|c|}
\hline County-Set & $\begin{array}{l}\text { Expected } \\
\text { Deaths }\end{array}$ & $\begin{array}{l}\text { Expected } \\
\text { Deaths } 95 \% \text { CI }\end{array}$ & $\begin{array}{l}\text { Observed } \\
\text { Deaths }\end{array}$ & $\begin{array}{l}\text { Observed/ } \\
\text { Expected }\end{array}$ & $\begin{array}{l}\text { Excess } \\
\text { Deaths }\end{array}$ & $\begin{array}{l}\text { Excess Deaths } \\
95 \% \text { CI }\end{array}$ & $\begin{array}{l}\text { Excess Deaths } \\
\text { Per } 100,000\end{array}$ & $\begin{array}{l}\text { COVID-19 } \\
\text { Deaths }\end{array}$ & $\begin{array}{c}\text { COVID-19 to } \\
\text { Excess Ratio (\%) }\end{array}$ \\
\hline Cass, MO & 1071 & $(996,1153)$ & 1119 & 1.04 & 48 & $(-34,123)$ & 44 & 83 & 172.9 \\
\hline Christian, MO & 770 & $(708,837)$ & 857 & 1.11 & 87 & $(20,149)$ & 96 & 87 & 100.0 \\
\hline Clay, MO & 1968 & $(1862,2074)$ & 2215 & 1.13 & 247 & $(141,353)$ & 97 & 241 & 97.6 \\
\hline Cole, MO & 722 & $(661,787)$ & 882 & 1.22 & 160 & $(95,221)$ & 208 & 138 & 86.2 \\
\hline $\begin{array}{l}\text { Crawford-Iron-Reynolds- } \\
\text { Washington, MO }\end{array}$ & 911 & $(837,985)$ & 1012 & 1.11 & 101 & $(27,175)$ & 156 & 95 & 94.1 \\
\hline Dallas-Hickory-Polk, MO & 793 & $(726,861)$ & 886 & 1.12 & 93 & $(25,160)$ & 155 & 101 & 108.6 \\
\hline $\begin{array}{l}\text { DeKalb and Nearby Coun- } \\
\text { ties, MO }\end{array}$ & 839 & $(770,910)$ & 962 & 1.15 & 123 & $(52,192)$ & 183 & 129 & 104.9 \\
\hline Dent-Phelps, MO & 693 & $(633,757)$ & 859 & 1.24 & 166 & $(102,226)$ & 276 & 100 & 60.2 \\
\hline $\begin{array}{l}\text { Douglas-Ozark-Texas- } \\
\text { Wright, MO }\end{array}$ & 957 & $(880,1037)$ & 993 & 1.04 & 36 & $(-44,113)$ & 54 & 92 & 255.6 \\
\hline Dunklin-Pemiscot, MO & 671 & $(607,733)$ & 862 & 1.28 & 191 & $(129,255)$ & 440 & 92 & 48.2 \\
\hline Franklin, MO & 1109 & $(1023,1193)$ & 1281 & 1.16 & 172 & $(88,258)$ & 165 & 149 & 86.6 \\
\hline $\begin{array}{l}\text { Gasconade-Montgomery- } \\
\text { Warren, MO }\end{array}$ & 742 & $(670,812)$ & 837 & 1.13 & 95 & $(25,167)$ & 154 & 76 & 80.0 \\
\hline Greene, MO & 3145 & $(3004,3293)$ & 3415 & 1.09 & 270 & $(122,411)$ & 91 & 378 & 140.0 \\
\hline $\begin{array}{l}\text { Grundy and Nearby Coun- } \\
\text { ties, MO }\end{array}$ & 670 & $(610,731)$ & 773 & 1.15 & 103 & $(42,163)$ & 202 & 114 & 110.7 \\
\hline $\begin{array}{l}\text { Howell-Oregon-Shannon, } \\
\text { MO }\end{array}$ & 785 & $(720,858)$ & 944 & 1.20 & 159 & $(86,224)$ & 271 & 114 & 71.7 \\
\hline Jackson, MO & 6525 & $(6306,6746)$ & 7542 & 1.16 & 1017 & $(796,1236)$ & 144 & 545 & 53.6 \\
\hline Jasper, MO & 1266 & $(1176,1356)$ & 1505 & 1.19 & 239 & $(149,329)$ & 197 & 191 & 79.9 \\
\hline Jefferson, MO & 2270 & $(2144,2398)$ & 2468 & 1.09 & 198 & $(70,324)$ & 88 & 221 & 111.6 \\
\hline Johnson, MO & 425 & $(379,473)$ & 494 & 1.16 & 69 & $(21,115)$ & 128 & 51 & 73.9 \\
\hline Laclede-Webster, MO & 898 & $(831,968)$ & 929 & 1.03 & 31 & $(-39,98)$ & 41 & 112 & 361.3 \\
\hline Lincoln-Pike, MO & 775 & $(711,839)$ & 791 & 1.02 & 16 & $(-48,80)$ & 20 & 79 & 493.8 \\
\hline Maries-Osage-Pulaski, MO & 605 & $(552,661)$ & 706 & 1.17 & 101 & $(45,154)$ & 135 & 69 & 68.3 \\
\hline $\begin{array}{l}\text { Marion-Monroe-Ralls- } \\
\text { Shelby, MO }\end{array}$ & 660 & $(599,726)$ & 748 & 1.13 & 88 & $(22,149)$ & 164 & 81 & 92.0 \\
\hline $\begin{array}{l}\text { Mississippi-New Madrid- } \\
\text { Scott, MO }\end{array}$ & 882 & $(804,952)$ & 1119 & 1.27 & 237 & $(167,315)$ & 350 & 123 & 51.9 \\
\hline Newton, MO & 702 & $(641,764)$ & 748 & 1.07 & 46 & $(-16,107)$ & 80 & 87 & 189.1 \\
\hline Pettis-Saline, MO & 745 & $(680,811)$ & 904 & 1.21 & 159 & $(93,224)$ & 243 & 120 & 75.5 \\
\hline Platte, MO & 800 & $(736,868)$ & 874 & 1.09 & 74 & $(6,138)$ & 70 & 78 & 105.4 \\
\hline $\begin{array}{l}\text { Randolph and Nearby } \\
\text { Counties, MO }\end{array}$ & 920 & $(849,996)$ & 1055 & 1.15 & 135 & $(59,206)$ & 180 & 108 & 80.0 \\
\hline St. Charles, MO & 4361 & $(4191,4532)$ & 4971 & 1.14 & 610 & $(439,780)$ & 124 & 552 & 90.5 \\
\hline St. Louis (city), MO & 3001 & $(2857,3145)$ & 3646 & 1.21 & 645 & $(501,789)$ & 220 & 362 & 56.1 \\
\hline St. Louis (county), MO & 10946 & $(10607,11277)$ & 12525 & 1.14 & 1579 & $(1248,1918)$ & 158 & 1485 & 94.0 \\
\hline $\begin{array}{l}\text { Scotland and Nearby } \\
\text { Counties, MO }\end{array}$ & 595 & $(533,655)$ & 634 & 1.07 & 39 & $(-21,101)$ & 71 & 58 & 148.7 \\
\hline Stone-Taney, MO & 1125 & $(1047,1211)$ & 1172 & 1.04 & 47 & $(-39,125)$ & 52 & 122 & 259.6 \\
\hline $\begin{array}{l}\text { Vernon and Nearby Coun- } \\
\text { ties, MO }\end{array}$ & 945 & $(867,1023)$ & 983 & 1.04 & 38 & $(-40,116)$ & 53 & 115 & 302.6 \\
\hline $\begin{array}{l}\text { Beaverhead-Gallatin- } \\
\text { Madison, MT }\end{array}$ & 785 & $(716,852)$ & 851 & 1.08 & 66 & $(-1,135)$ & 48 & 54 & 81.8 \\
\hline Cascade, MT & 949 & $(879,1026)$ & 1078 & 1.14 & 129 & $(52,199)$ & 158 & 146 & 113.2 \\
\hline $\begin{array}{l}\text { Deer Lodge and Nearby } \\
\text { Counties, MT }\end{array}$ & 1187 & $(1102,1276)$ & 1303 & 1.10 & 116 & $(27,201)$ & 117 & 115 & 99.1 \\
\hline Eastern Montana, MT & 1004 & $(925,1078)$ & 1320 & 1.31 & 316 & $(242,395)$ & 349 & 279 & 88.3 \\
\hline Flathead, MT & 1017 & $(937,1093)$ & 1137 & 1.12 & 120 & $(44,200)$ & 113 & 74 & 61.7 \\
\hline $\begin{array}{l}\text { Jefferson-Lewis and Clark, } \\
\text { MT }\end{array}$ & 787 & $(725,851)$ & 865 & 1.10 & 78 & $(14,140)$ & 94 & 57 & 73.1 \\
\hline $\begin{array}{l}\text { Lake-Lincoln-Mineral- } \\
\text { Sanders, MT }\end{array}$ & 831 & $(764,896)$ & 911 & 1.10 & 80 & $(15,147)$ & 118 & 61 & 76.2 \\
\hline
\end{tabular}


Table C1: Primary Estimates for Each County-Set

\begin{tabular}{|c|c|c|c|c|c|c|c|c|c|}
\hline County-Set & $\begin{array}{l}\text { Expected } \\
\text { Deaths }\end{array}$ & $\begin{array}{l}\text { Expected } \\
\text { Deaths } 95 \% \mathrm{CI}\end{array}$ & $\begin{array}{l}\text { Observed } \\
\text { Deaths }\end{array}$ & $\begin{array}{l}\text { Observed/ } \\
\text { Expected }\end{array}$ & $\begin{array}{l}\text { Excess } \\
\text { Deaths }\end{array}$ & $\begin{array}{l}\text { Excess Deaths } \\
95 \% \mathrm{CI}\end{array}$ & $\begin{array}{l}\text { Excess Deaths } \\
\text { Per } 100,000\end{array}$ & $\begin{array}{l}\text { COVID-19 } \\
\text { Deaths }\end{array}$ & $\begin{array}{c}\text { COVID-19 to } \\
\text { Excess Ratio (\%) }\end{array}$ \\
\hline Missoula, MT & 954 & $(877,1025)$ & 999 & 1.05 & 45 & $(-26,122)$ & 37 & 68 & 151.1 \\
\hline $\begin{array}{l}\text { North Central Montana, } \\
\text { MT }\end{array}$ & 822 & $(754,895)$ & 985 & 1.20 & 163 & $(90,231)$ & 216 & 167 & 102.5 \\
\hline $\begin{array}{l}\text { South Central Montana, } \\
\text { MT }\end{array}$ & 588 & $(529,645)$ & 676 & 1.15 & 88 & $(31,147)$ & 152 & 55 & 62.5 \\
\hline Yellowstone, MT & 1669 & $(1564,1772)$ & 1897 & 1.14 & 228 & $(125,333)$ & 141 & 198 & 86.8 \\
\hline $\begin{array}{l}\text { Banner and Nearby Coun- } \\
\text { ties, NE }\end{array}$ & 644 & $(583,707)$ & 811 & 1.26 & 167 & $(104,228)$ & 315 & 142 & 85.0 \\
\hline $\begin{array}{l}\text { Buffalo-Kearney-Sherman, } \\
\text { NE }\end{array}$ & 494 & $(447,541)$ & 586 & 1.19 & 92 & $(45,139)$ & 154 & 74 & 80.4 \\
\hline $\begin{array}{l}\text { Burt-Dakota-Thurston- } \\
\text { Washington, NE }\end{array}$ & 568 & $(510,625)$ & 663 & 1.17 & 95 & $(38,153)$ & 172 & 115 & 121.1 \\
\hline $\begin{array}{l}\text { Cuming and Nearby Coun- } \\
\text { ties, NE }\end{array}$ & 447 & $(396,499)$ & 530 & 1.19 & 83 & $(31,134)$ & 168 & 86 & 103.6 \\
\hline Dodge-Saunders, NE & 695 & $(630,760)$ & 813 & 1.17 & 118 & $(53,183)$ & 202 & 81 & 68.6 \\
\hline Douglas, NE & 4324 & $(4140,4492)$ & 4936 & 1.14 & 612 & $(444,796)$ & 106 & 550 & 89.9 \\
\hline $\begin{array}{l}\text { Frontier and Nearby Coun- } \\
\text { ties, NE }\end{array}$ & 536 & $(483,592)$ & 614 & 1.15 & 78 & $(22,131)$ & 160 & 83 & 106.4 \\
\hline Hall, NE & 590 & $(536,650)$ & 630 & 1.07 & 40 & $(-20,94)$ & 64 & 114 & 285.0 \\
\hline $\begin{array}{l}\text { Howard-Merrick-Nance- } \\
\text { Platte, NE }\end{array}$ & 533 & $(482,589)$ & 591 & 1.11 & 58 & $(2,109)$ & 114 & 95 & 163.8 \\
\hline Lancaster, NE & 2416 & $(2290,2540)$ & 2560 & 1.06 & 144 & $(20,270)$ & 44 & 173 & 120.1 \\
\hline $\begin{array}{l}\text { Lincoln and Nearby Coun- } \\
\text { ties, NE }\end{array}$ & 561 & $(510,619)$ & 632 & 1.13 & 71 & $(13,122)$ & 145 & 92 & 129.6 \\
\hline Northwest Nebraska, NE & 624 & $(564,682)$ & 729 & 1.17 & 105 & $(47,165)$ & 199 & 97 & 92.4 \\
\hline $\begin{array}{l}\text { Saline and Nearby Coun- } \\
\text { ties, NE }\end{array}$ & 745 & $(682,811)$ & 770 & 1.03 & 25 & $(-41,88)$ & 43 & 68 & 272.0 \\
\hline Sarpy, NE & 1137 & $(1055,1220)$ & 1313 & 1.15 & 176 & $(93,258)$ & 92 & 132 & 75.0 \\
\hline $\begin{array}{l}\text { South Central Nebraska, } \\
\text { NE }\end{array}$ & 714 & $(651,776)$ & 815 & 1.14 & 101 & $(39,164)$ & 166 & 100 & 99.0 \\
\hline Southeast Nebraska, NE & 735 & $(669,804)$ & 840 & 1.14 & 105 & $(36,171)$ & 161 & 72 & 68.6 \\
\hline $\begin{array}{l}\text { Wheeler and Nearby Coun- } \\
\text { ties, NE }\end{array}$ & 1017 & $(945,1093)$ & 1041 & 1.02 & 24 & $(-52,96)$ & 29 & 145 & 604.2 \\
\hline $\begin{array}{l}\text { York and Nearby Counties, } \\
\text { NE }\end{array}$ & 614 & $(557,674)$ & 666 & 1.08 & 52 & $(-8,109)$ & 96 & 87 & 167.3 \\
\hline Carson City & 1443 & $(1341,1540)$ & 1542 & 1.07 & 99 & $(2,201)$ & 93 & 118 & 119.2 \\
\hline Douglas, NV & & & & & & & & & \\
\hline Clark-Nye, NV & 19372 & $(18897,19800)$ & 22168 & 1.14 & 2796 & $(2368,3271)$ & 118 & 2674 & 95.6 \\
\hline $\begin{array}{l}\text { Lyon and Nearby Counties, } \\
\text { NV }\end{array}$ & 1160 & $(1079,1247)$ & 1346 & 1.16 & 186 & $(99,267)$ & 197 & 121 & 65.1 \\
\hline $\begin{array}{l}\text { Northern Nevada, exclud- } \\
\text { ing Washoe, NV }\end{array}$ & 737 & $(673,800)$ & 834 & 1.13 & 97 & $(34,161)$ & 98 & 79 & 81.4 \\
\hline Washoe, NV & 4250 & $(4081,4418)$ & 4727 & 1.11 & 477 & $(309,646)$ & 99 & 470 & 98.5 \\
\hline Belknap-Carroll, NH & 1451 & $(1357,1543)$ & 1408 & 0.97 & -43 & $(-135,51)$ & -39 & 52 & NA \\
\hline Cheshire, NH & 800 & $(735,869)$ & 773 & 0.97 & -27 & $(-96,38)$ & -35 & 11 & NA \\
\hline Coos-Grafton, NH & 1402 & $(1309,1491)$ & 1362 & 0.97 & -40 & $(-129,53)$ & -33 & 35 & NA \\
\hline Hillsborough, NH & 3738 & $(3583,3897)$ & 4004 & 1.07 & 266 & $(107,421)$ & 64 & 456 & 171.4 \\
\hline Merrimack-Sullivan, $\mathrm{NH}$ & 2029 & $(1923,2143)$ & 2008 & 0.99 & -21 & $(-135,85)$ & -11 & 82 & NA \\
\hline Rockingham, NH & 2751 & $(2625,2885)$ & 2924 & 1.06 & 173 & $(39,299)$ & 55 & 196 & 113.3 \\
\hline Strafford, NH & 1298 & $(1210,1386)$ & 1219 & 0.94 & -79 & $(-167,9)$ & -60 & 31 & NA \\
\hline Atlantic, NJ & 2918 & $(2779,3055)$ & 3313 & 1.14 & 395 & $(258,534)$ & 150 & 338 & 85.6 \\
\hline Bergen, NJ & 7446 & $(7209,7677)$ & 9341 & 1.25 & 1895 & $(1664,2132)$ & 201 & 2089 & 110.2 \\
\hline Burlington, NJ & 4367 & $(4199,4544)$ & 4799 & 1.10 & 432 & $(255,600)$ & 97 & 629 & 145.6 \\
\hline Camden, NJ & 5151 & $(4950,5351)$ & 5891 & 1.14 & 740 & $(540,941)$ & 146 & 807 & 109.1 \\
\hline Cape May, NJ & 1338 & $(1246,1435)$ & 1435 & 1.07 & 97 & $(0,189)$ & 106 & 137 & 141.2 \\
\hline Cumberland, NJ & 1692 & $(1586,1793)$ & 1912 & 1.13 & 220 & $(119,326)$ & 147 & 202 & 91.8 \\
\hline
\end{tabular}


Table C1: Primary Estimates for Each County-Set

\begin{tabular}{|c|c|c|c|c|c|c|c|c|c|}
\hline County-Set & $\begin{array}{l}\text { Expected } \\
\text { Deaths }\end{array}$ & $\begin{array}{l}\text { Expected } \\
\text { Deaths } 95 \% \text { CI }\end{array}$ & $\begin{array}{l}\text { Observed } \\
\text { Deaths }\end{array}$ & $\begin{array}{l}\text { Observed/ } \\
\text { Expected }\end{array}$ & $\begin{array}{l}\text { Excess } \\
\text { Deaths }\end{array}$ & $\begin{array}{l}\text { Excess Deaths } \\
95 \% \mathrm{CI}\end{array}$ & $\begin{array}{l}\text { Excess Deaths } \\
\text { Per } 100,000\end{array}$ & $\begin{array}{l}\text { COVID-19 } \\
\text { Deaths }\end{array}$ & $\begin{array}{l}\text { COVID-19 to } \\
\text { Excess Ratio (\%) }\end{array}$ \\
\hline Essex, NJ & 6128 & $(5931,6331)$ & 8771 & 1.43 & 2643 & $(2440,2840)$ & 328 & 2181 & 82.5 \\
\hline Gloucester, NJ & 2898 & $(2760,3058)$ & 3309 & 1.14 & 411 & $(251,549)$ & 141 & 389 & 94.6 \\
\hline Hudson, NJ & 3763 & $(3617,3922)$ & 5807 & 1.54 & 2044 & $(1885,2190)$ & 300 & 1603 & 78.4 \\
\hline Hunterdon, NJ & 956 & $(889,1035)$ & 1109 & 1.16 & 153 & $(74,220)$ & 123 & 163 & 106.5 \\
\hline Mercer, NJ & 3013 & $(2876,3145)$ & 3771 & 1.25 & 758 & $(626,895)$ & 204 & 663 & 87.5 \\
\hline Middlesex, NJ & 6209 & $(6015,6412)$ & 7833 & 1.26 & 1624 & $(1421,1818)$ & 195 & 1543 & 95.0 \\
\hline Monmouth, NJ & 5777 & $(5581,5988)$ & 6942 & 1.20 & 1165 & $(954,1361)$ & 188 & 1016 & 87.2 \\
\hline Morris, NJ & 4039 & $(3881,4188)$ & 4991 & 1.24 & 952 & $(803,1110)$ & 193 & 896 & 94.1 \\
\hline Ocean, NJ & 7549 & $(7290,7822)$ & 8673 & 1.15 & 1124 & $(851,1383)$ & 183 & 1276 & 113.5 \\
\hline Passaic, NJ & 3835 & $(3670,3990)$ & 5453 & 1.42 & 1618 & $(1463,1783)$ & 322 & 1438 & 88.9 \\
\hline Salem, NJ & 775 & $(707,845)$ & 936 & 1.21 & 161 & $(91,229)$ & 259 & 99 & 61.5 \\
\hline Somerset, NJ & 2550 & $(2424,2670)$ & 3066 & 1.20 & 516 & $(396,642)$ & 155 & 582 & 112.8 \\
\hline Sussex, NJ & 1333 & $(1242,1417)$ & 1553 & 1.17 & 220 & $(136,311)$ & 157 & 233 & 105.9 \\
\hline Union, NJ & 4050 & $(3899,4227)$ & 5808 & 1.43 & 1758 & $(1581,1909)$ & 312 & 1479 & 84.1 \\
\hline Warren, NJ & 1074 & $(998,1158)$ & 1252 & 1.17 & 178 & $(94,254)$ & 168 & 164 & 92.1 \\
\hline Bernalillo, NM & 6192 & $(5975,6401)$ & 7192 & 1.16 & 1000 & $(791,1217)$ & 147 & 655 & 65.5 \\
\hline $\begin{array}{l}\text { Catron-Cibola-Sierra- } \\
\text { Socorro, NM }\end{array}$ & 741 & $(682,807)$ & 941 & 1.27 & 200 & $(134,259)$ & 350 & 165 & 82.5 \\
\hline Chaves, NM & 677 & $(616,743)$ & 849 & 1.25 & 172 & $(106,233)$ & 270 & 120 & 69.8 \\
\hline Curry-Roosevelt, NM & 588 & $(537,647)$ & 664 & 1.13 & 76 & $(17,127)$ & 112 & 100 & 131.6 \\
\hline Doña Ana, NM & 1702 & $(1605,1798)$ & 2291 & 1.35 & 589 & $(493,686)$ & 267 & 340 & 57.7 \\
\hline Eddy, NM & 610 & $(550,671)$ & 636 & 1.04 & 26 & $(-35,86)$ & 44 & 91 & 350.0 \\
\hline Grant-Hidalgo-Luna, NM & 729 & $(667,792)$ & 893 & 1.22 & 164 & $(101,226)$ & 301 & 90 & 54.9 \\
\hline $\begin{array}{l}\text { Guadalupe and Nearby } \\
\text { Counties, NM }\end{array}$ & 638 & $(577,701)$ & 677 & 1.06 & 39 & $(-24,100)$ & 79 & 52 & 133.3 \\
\hline Lea, NM & 574 & $(521,630)$ & 714 & 1.24 & 140 & $(84,193)$ & 198 & 139 & 99.3 \\
\hline Los Alamos-Santa Fe, NM & 1441 & $(1350,1533)$ & 1600 & 1.11 & 159 & $(67,250)$ & 93 & 97 & 61.0 \\
\hline McKinley, NM & 745 & $(681,810)$ & 1192 & 1.60 & 447 & $(382,511)$ & 625 & 387 & 86.6 \\
\hline $\begin{array}{l}\text { Northeast New Mexico, } \\
\text { NM }\end{array}$ & 545 & $(492,601)$ & 603 & 1.11 & 58 & $(2,111)$ & 120 & 56 & 96.6 \\
\hline Otero, NM & 678 & $(615,739)$ & 756 & 1.12 & 78 & $(17,141)$ & 115 & 47 & 60.3 \\
\hline Rio Arriba-Taos, NM & 799 & $(736,867)$ & 919 & 1.15 & 120 & $(52,183)$ & 168 & 95 & 79.2 \\
\hline Sandoval, NM & 1331 & $(1244,1417)$ & 1431 & 1.08 & 100 & $(14,187)$ & 66 & 158 & 158.0 \\
\hline San Juan, NM & 1182 & $(1098,1267)$ & 1538 & 1.30 & 356 & $(271,440)$ & 293 & 345 & 96.9 \\
\hline Valencia, NM & 770 & $(702,836)$ & 853 & 1.11 & 83 & $(17,151)$ & 107 & 75 & 90.4 \\
\hline Albany-Greene, NY & 3352 & $(3209,3506)$ & 3688 & 1.10 & 336 & $(182,479)$ & 95 & 273 & 81.2 \\
\hline Allegany-Steuben, NY & 1578 & $(1480,1677)$ & 1726 & 1.09 & 148 & $(49,246)$ & 105 & 204 & 137.8 \\
\hline Bronx, NY & 9692 & $(9407,9973)$ & 15933 & 1.64 & 6241 & $(5960,6526)$ & 441 & 4631 & 74.2 \\
\hline Broome, NY & 2138 & $(2013,2253)$ & 2406 & 1.13 & 268 & $(153,393)$ & 142 & 242 & 90.3 \\
\hline $\begin{array}{l}\text { Cattaraugus-Wyoming, } \\
\text { NY }\end{array}$ & 1290 & $(1199,1377)$ & 1386 & 1.07 & 96 & $(9,187)$ & 83 & 84 & 87.5 \\
\hline Cayuga, NY & 795 & $(732,859)$ & 897 & 1.13 & 102 & $(38,165)$ & 134 & 31 & 30.4 \\
\hline Chautauqua, NY & 1574 & $(1479,1671)$ & 1568 & 1.00 & -6 & $(-103,89)$ & -5 & 44 & NA \\
\hline Chemung, NY & 1076 & $(998,1162)$ & 1115 & 1.04 & 39 & $(-47,117)$ & 48 & 115 & 294.9 \\
\hline Chenango-Cortland, NY & 1083 & $(995,1163)$ & 1155 & 1.07 & 72 & $(-8,160)$ & 76 & 66 & 91.7 \\
\hline Clinton, NY & 762 & $(699,829)$ & 806 & 1.06 & 44 & $(-23,107)$ & 54 & 10 & 22.7 \\
\hline Columbia, NY & 697 & $(636,763)$ & 696 & 1.00 & -1 & $(-67,60)$ & -1 & 50 & NA \\
\hline Delaware-Schoharie, NY & 864 & $(796,933)$ & 907 & 1.05 & 43 & $(-26,111)$ & 58 & 28 & 65.1 \\
\hline Dutchess, NY & 2686 & $(2554,2818)$ & 2947 & 1.10 & 261 & $(129,393)$ & 89 & 291 & 111.5 \\
\hline Erie, NY & 9945 & $(9653,10245)$ & 11162 & 1.12 & 1217 & $(917,1509)$ & 132 & 1300 & 106.8 \\
\hline Essex-Franklin, NY & 930 & $(858,1008)$ & 958 & 1.03 & 28 & $(-50,100)$ & 32 & 25 & 89.3 \\
\hline Fulton-Hamilton, NY & 670 & $(608,738)$ & 752 & 1.12 & 82 & $(14,144)$ & 143 & 57 & 69.5 \\
\hline Genesee-Orleans, NY & 1084 & $(1003,1160)$ & 1222 & 1.13 & 138 & $(62,219)$ & 142 & 121 & 87.7 \\
\hline Herkimer, NY & 703 & $(643,768)$ & 715 & 1.02 & 12 & $(-53,72)$ & 20 & 42 & 350.0 \\
\hline Jefferson-Lewis, NY & 1217 & $(1136,1299)$ & 1196 & 0.98 & -21 & $(-103,60)$ & -15 & 23 & NA \\
\hline Kings, NY & 15687 & $(15280,16112)$ & 24874 & 1.59 & 9187 & $(8762,9594)$ & 359 & 7138 & 77.7 \\
\hline
\end{tabular}


Table C1: Primary Estimates for Each County-Set

\begin{tabular}{|c|c|c|c|c|c|c|c|c|c|}
\hline County-Set & $\begin{array}{l}\text { Expected } \\
\text { Deaths }\end{array}$ & $\begin{array}{l}\text { Expected } \\
\text { Deaths } 95 \% \text { CI }\end{array}$ & $\begin{array}{l}\text { Observed } \\
\text { Deaths }\end{array}$ & $\begin{array}{l}\text { Observed/ } \\
\text { Expected }\end{array}$ & $\begin{array}{l}\text { Excess } \\
\text { Deaths }\end{array}$ & $\begin{array}{l}\text { Excess Deaths } \\
95 \% \mathrm{CI}\end{array}$ & $\begin{array}{c}\text { Excess Deaths } \\
\text { Per } 100,000\end{array}$ & $\begin{array}{l}\text { COVID-19 } \\
\text { Deaths }\end{array}$ & $\begin{array}{l}\text { COVID-19 to } \\
\text { Excess Ratio (\%) }\end{array}$ \\
\hline Livingston, NY & 600 & $(544,662)$ & 590 & 0.98 & -10 & $(-72,46)$ & -15 & 36 & NA \\
\hline Madison, NY & 603 & $(545,660)$ & 725 & 1.20 & 122 & $(65,180)$ & 173 & 74 & 60.7 \\
\hline Monroe, NY & 6931 & $(6709,7159)$ & 7791 & 1.12 & 860 & $(632,1082)$ & 116 & 729 & 84.8 \\
\hline Montgomery, NY & 598 & $(541,661)$ & 664 & 1.11 & 66 & $(3,123)$ & 133 & 39 & 59.1 \\
\hline Nassau, NY & 11020 & $(10701,11316)$ & 14273 & 1.30 & 3253 & $(2957,3572)$ & 239 & 3004 & 92.3 \\
\hline New York (county), NY & 10036 & $(9765,10325)$ & 14013 & 1.40 & 3977 & $(3688,4248)$ & 245 & 3073 & 77.3 \\
\hline Niagara, NY & 2471 & $(2343,2596)$ & 2808 & 1.14 & 337 & $(212,465)$ & 161 & 262 & 77.7 \\
\hline Oneida, NY & 2650 & $(2516,2784)$ & 2942 & 1.11 & 292 & $(158,426)$ & 128 & 272 & 93.2 \\
\hline Onondaga, NY & 4493 & $(4326,4685)$ & 5119 & 1.14 & 626 & $(434,793)$ & 136 & 527 & 84.2 \\
\hline Ontario, NY & 1120 & $(1048,1198)$ & 1122 & 1.00 & 2 & $(-76,74)$ & 2 & 80 & 4000.0 \\
\hline Orange, NY & 2879 & $(2759,3004)$ & 3516 & 1.22 & 637 & $(512,757)$ & 165 & 628 & 98.6 \\
\hline Oswego, NY & 1144 & $(1067,1222)$ & 1181 & 1.03 & 37 & $(-41,114)$ & 31 & 60 & 162.2 \\
\hline Otsego, NY & 647 & $(588,708)$ & 691 & 1.07 & 44 & $(-17,103)$ & 74 & 21 & 47.7 \\
\hline Putnam, NY & 741 & $(683,807)$ & 896 & 1.21 & 155 & $(89,213)$ & 157 & 129 & 83.2 \\
\hline Queens, NY & 14497 & $(14123,14889)$ & 23062 & 1.59 & 8565 & $(8173,8939)$ & 382 & 7040 & 82.2 \\
\hline Rensselaer, NY & 1495 & $(1391,1590)$ & 1662 & 1.11 & 167 & $(72,271)$ & 104 & 87 & 52.1 \\
\hline Richmond, NY & 3896 & $(3734,4059)$ & 5041 & 1.29 & 1145 & $(982,1307)$ & 240 & 1174 & 102.5 \\
\hline Rockland, NY & 2309 & $(2195,2421)$ & 3221 & 1.39 & 912 & $(800,1026)$ & 279 & 873 & 95.7 \\
\hline St. Lawrence, NY & 1014 & $(935,1088)$ & 1117 & 1.10 & 103 & $(29,182)$ & 96 & 49 & 47.6 \\
\hline Saratoga, NY & 2058 & $(1948,2167)$ & 2051 & 1.00 & -7 & $(-116,103)$ & -3 & 70 & NA \\
\hline Schenectady, NY & 1627 & $(1534,1728)$ & 1746 & 1.07 & 119 & $(18,212)$ & 76 & 97 & 81.5 \\
\hline Schuyler-Seneca-Yates, NY & 902 & $(829,977)$ & 914 & 1.01 & 12 & $(-63,85)$ & 16 & 61 & 508.3 \\
\hline Suffolk, NY & 12974 & $(12618,13297)$ & 15765 & 1.22 & 2791 & $(2468,3147)$ & 189 & 2991 & 107.2 \\
\hline Sullivan, NY & 753 & $(689,818)$ & 888 & 1.18 & 135 & $(70,199)$ & 177 & 71 & 52.6 \\
\hline Tioga, NY & 492 & $(437,545)$ & 579 & 1.18 & 87 & $(34,142)$ & 180 & 74 & 85.1 \\
\hline Tompkins, NY & 729 & $(664,794)$ & 704 & 0.97 & -25 & $(-90,40)$ & -25 & 29 & NA \\
\hline Ulster, NY & 1845 & $(1746,1948)$ & 1992 & 1.08 & 147 & $(44,246)$ & 82 & 161 & 109.5 \\
\hline Warren, NY & 786 & $(717,858)$ & 790 & 1.01 & 4 & $(-68,73)$ & 6 & 47 & 1175.0 \\
\hline Washington, NY & 677 & $(616,742)$ & 755 & 1.12 & 78 & $(13,139)$ & 128 & 17 & 21.8 \\
\hline Wayne, NY & 940 & $(867,1011)$ & 969 & 1.03 & 29 & $(-42,102)$ & 32 & 56 & 193.1 \\
\hline Westchester, NY & 7251 & $(7023,7492)$ & 9629 & 1.33 & 2378 & $(2137,2606)$ & 247 & 2050 & 86.2 \\
\hline Alamance, NC & 1841 & $(1733,1952)$ & 2037 & 1.11 & 196 & $(85,304)$ & 114 & 158 & 80.6 \\
\hline Alexander-Catawba, NC & 2232 & $(2107,2356)$ & 2503 & 1.12 & 271 & $(147,396)$ & 137 & 237 & 87.5 \\
\hline $\begin{array}{l}\text { Alleghany-Ashe-Avery- } \\
\text { Watauga, NC }\end{array}$ & 1120 & $(1033,1203)$ & 1234 & 1.10 & 114 & $(31,201)$ & 99 & 88 & 77.2 \\
\hline Anson-Richmond, NC & 939 & $(865,1012)$ & 995 & 1.06 & 56 & $(-17,130)$ & 80 & 93 & 166.1 \\
\hline $\begin{array}{l}\text { Beaufort-Martin-Pamlico, } \\
\text { NC }\end{array}$ & 1163 & $(1080,1249)$ & 1254 & 1.08 & 91 & $(5,174)$ & 110 & 107 & 117.6 \\
\hline Bladen-Columbus, NC & 1177 & $(1096,1266)$ & 1379 & 1.17 & 202 & $(113,283)$ & 230 & 119 & 58.9 \\
\hline Brunswick, NC & 1738 & $(1647,1838)$ & 1777 & 1.02 & 39 & $(-61,130)$ & 26 & 88 & 225.6 \\
\hline Buncombe-Madison, NC & 3122 & $(2981,3270)$ & 3307 & 1.06 & 185 & $(37,326)$ & 65 & 209 & 113.0 \\
\hline Burke, NC & 1073 & $(993,1145)$ & 1203 & 1.12 & 130 & $(58,210)$ & 143 & 97 & 74.6 \\
\hline Cabarrus, NC & 1670 & $(1570,1772)$ & 1877 & 1.12 & 207 & $(105,307)$ & 94 & 143 & 69.1 \\
\hline Caldwell, NC & 1038 & $(957,1120)$ & 1156 & 1.11 & 118 & $(36,199)$ & 144 & 118 & 100.0 \\
\hline $\begin{array}{l}\text { Camden-Currituck- } \\
\text { Pasquotank, NC }\end{array}$ & 794 & $(726,858)$ & 936 & 1.18 & 142 & $(78,210)$ & 179 & 64 & 45.1 \\
\hline Carteret, NC & 929 & $(861,1006)$ & 881 & 0.95 & -48 & $(-125,20)$ & -68 & 35 & NA \\
\hline Caswell-Person, NC & 778 & $(711,849)$ & 868 & 1.12 & 90 & $(19,157)$ & 143 & 45 & 50.0 \\
\hline Chatham, NC & 781 & $(717,849)$ & 842 & 1.08 & 61 & $(-7,125)$ & 79 & 58 & 95.1 \\
\hline $\begin{array}{l}\text { Cherokee-Clay-Graham- } \\
\text { Swain, NC }\end{array}$ & 912 & $(837,988)$ & 956 & 1.05 & 44 & $(-32,119)$ & 70 & 57 & 129.5 \\
\hline Cleveland, NC & 1320 & $(1233,1414)$ & 1484 & 1.12 & 164 & $(70,251)$ & 166 & 172 & 104.9 \\
\hline Craven, NC & 1211 & $(1126,1303)$ & 1359 & 1.12 & 148 & $(56,233)$ & 143 & 105 & 70.9 \\
\hline Cumberland, NC & 2770 & $(2629,2900)$ & 3054 & 1.10 & 284 & $(154,425)$ & 85 & 158 & 55.6 \\
\hline Dare-Hyde-Tyrrell- & 691 & $(630,753)$ & 687 & 0.99 & -4 & $(-66,57)$ & -7 & 28 & NA \\
\hline
\end{tabular}


Table C1: Primary Estimates for Each County-Set

\begin{tabular}{|c|c|c|c|c|c|c|c|c|c|}
\hline County-Set & $\begin{array}{l}\text { Expected } \\
\text { Deaths }\end{array}$ & $\begin{array}{l}\text { Expected } \\
\text { Deaths } 95 \% \text { CI }\end{array}$ & $\begin{array}{l}\text { Observed } \\
\text { Deaths }\end{array}$ & $\begin{array}{l}\text { Observed/ } \\
\text { Expected }\end{array}$ & $\begin{array}{l}\text { Excess } \\
\text { Deaths }\end{array}$ & $\begin{array}{l}\text { Excess Deaths } \\
95 \% \text { CI }\end{array}$ & $\begin{array}{l}\text { Excess Deaths } \\
\text { Per } 100,000\end{array}$ & $\begin{array}{l}\text { COVID-19 } \\
\text { Deaths }\end{array}$ & $\begin{array}{l}\text { COVID-19 to } \\
\text { Excess Ratio (\%) }\end{array}$ \\
\hline Davidson, NC & 1969 & $(1852,2077)$ & 2205 & 1.12 & 236 & $(128,353)$ & 140 & 148 & 62.7 \\
\hline Davie-Yadkin, NC & 1003 & $(927,1078)$ & 1043 & 1.04 & 40 & $(-35,116)$ & 49 & 79 & 197.5 \\
\hline Duplin, NC & 563 & $(508,624)$ & 704 & 1.25 & 141 & $(80,196)$ & 240 & 81 & 57.4 \\
\hline Durham, NC & 2211 & $(2095,2330)$ & 2432 & 1.10 & 221 & $(102,337)$ & 68 & 153 & 69.2 \\
\hline Edgecombe, $\mathrm{NC}$ & 746 & $(680,809)$ & 804 & 1.08 & 58 & $(-5,124)$ & 116 & 90 & 155.2 \\
\hline Forsyth-Stokes, NC & 4253 & $(4081,4431)$ & 4615 & 1.09 & 362 & $(184,534)$ & 84 & 298 & 82.3 \\
\hline Franklin-Warren, NC & 980 & $(903,1060)$ & 1015 & 1.04 & 35 & $(-45,112)$ & 39 & 62 & 177.1 \\
\hline Gaston, NC & 2533 & $(2405,2663)$ & 2785 & 1.10 & 252 & $(122,380)$ & 110 & 249 & 98.8 \\
\hline $\begin{array}{l}\text { Gates and Nearby Coun- } \\
\text { ties, NC }\end{array}$ & 1061 & $(985,1139)$ & 1254 & 1.18 & 193 & $(115,269)$ & 239 & 159 & 82.4 \\
\hline Granville-Vance, NC & 1201 & $(1116,1289)$ & 1366 & 1.14 & 165 & $(77,250)$ & 154 & 128 & 77.6 \\
\hline Greene-Jones-Lenoir, NC & 1106 & $(1024,1182)$ & 1308 & 1.18 & 202 & $(126,284)$ & 236 & 137 & 67.8 \\
\hline Guilford, NC & 4856 & $(4666,5036)$ & 5160 & 1.06 & 304 & $(124,494)$ & 56 & 391 & 128.6 \\
\hline Halifax-Northampton, NC & 951 & $(873,1028)$ & 1167 & 1.23 & 216 & $(139,294)$ & 316 & 105 & 48.6 \\
\hline Harnett, NC & 1115 & $(1041,1195)$ & 1230 & 1.10 & 115 & $(35,189)$ & 83 & 103 & 89.6 \\
\hline Haywood, NC & 903 & $(828,980)$ & 956 & 1.06 & 53 & $(-24,128)$ & 83 & 77 & 145.3 \\
\hline Henderson, NC & 1486 & $(1393,1580)$ & 1530 & 1.03 & 44 & $(-50,137)$ & 37 & 108 & 245.5 \\
\hline Hoke-Scotland, NC & 796 & $(734,865)$ & 1040 & 1.31 & 244 & $(175,306)$ & 271 & 112 & 45.9 \\
\hline Iredell, NC & 1657 & $(1557,1752)$ & 1871 & 1.13 & 214 & $(119,314)$ & 116 & 140 & 65.4 \\
\hline $\begin{array}{l}\text { Jackson-Macon- } \\
\text { Transylvania, NC }\end{array}$ & 1384 & $(1294,1483)$ & 1436 & 1.04 & 52 & $(-47,142)$ & 45 & 72 & 138.5 \\
\hline Johnston, NC & 1698 & $(1599,1803)$ & 1818 & 1.07 & 120 & $(15,219)$ & 56 & 134 & 111.7 \\
\hline Lee, NC & 659 & $(601,716)$ & 746 & 1.13 & 87 & $(30,145)$ & 138 & 51 & 58.6 \\
\hline Lincoln, NC & 895 & $(822,967)$ & 1012 & 1.13 & 117 & $(45,190)$ & 135 & 82 & 70.1 \\
\hline $\begin{array}{l}\text { McDowell-Mitchell- } \\
\text { Yancey, NC }\end{array}$ & 1050 & $(975,1129)$ & 1147 & 1.09 & 97 & $(18,172)$ & 123 & 118 & 121.6 \\
\hline Mecklenburg, NC & 6753 & $(6552,6985)$ & 7676 & 1.14 & 923 & $(691,1124)$ & 82 & 683 & 74.0 \\
\hline Montgomery-Moore, NC & 1535 & $(1431,1629)$ & 1687 & 1.10 & 152 & $(58,256)$ & 118 & 153 & 100.7 \\
\hline Nash, NC & 1166 & $(1082,1254)$ & 1235 & 1.06 & 69 & $(-19,153)$ & 73 & 128 & 185.5 \\
\hline New Hanover, NC & 2143 & $(2023,2256)$ & 2332 & 1.09 & 189 & $(76,309)$ & 79 & 92 & 48.7 \\
\hline Onslow, NC & 1425 & $(1342,1516)$ & 1429 & 1.00 & 4 & $(-87,87)$ & 2 & 82 & 2050.0 \\
\hline Orange, $\mathrm{NC}$ & 867 & $(790,935)$ & 904 & 1.04 & 37 & $(-31,114)$ & 25 & 58 & 156.8 \\
\hline Pender, NC & 671 & $(607,733)$ & 679 & 1.01 & 8 & $(-54,72)$ & 13 & 31 & 387.5 \\
\hline Pitt, NC & 1386 & $(1296,1476)$ & 1643 & 1.19 & 257 & $(167,347)$ & 141 & 139 & 54.1 \\
\hline Polk-Rutherford, NC & 1236 & $(1150,1320)$ & 1367 & 1.11 & 131 & $(47,217)$ & 148 & 146 & 111.5 \\
\hline Randolph, NC & 1704 & $(1596,1806)$ & 1866 & 1.10 & 162 & $(60,270)$ & 113 & 148 & 91.4 \\
\hline Robeson, NC & 1531 & $(1437,1630)$ & 1753 & 1.15 & 222 & $(123,316)$ & 170 & 202 & 91.0 \\
\hline Rockingham, NC & 1228 & $(1141,1318)$ & 1445 & 1.18 & 217 & $(127,304)$ & 240 & 115 & 53.0 \\
\hline Rowan, NC & 1735 & $(1628,1844)$ & 1971 & 1.14 & 236 & $(127,343)$ & 165 & 183 & 77.5 \\
\hline Sampson, NC & 762 & $(698,827)$ & 759 & 1.00 & -3 & $(-68,61)$ & -5 & 62 & NA \\
\hline Stanly, NC & 774 & $(705,839)$ & 853 & 1.10 & 79 & $(14,148)$ & 125 & 84 & 106.3 \\
\hline Surry, NC & 1025 & $(946,1098)$ & 1089 & 1.06 & 64 & $(-9,143)$ & 90 & 89 & 139.1 \\
\hline Union, $\mathrm{NC}$ & 1631 & $(1534,1731)$ & 1677 & 1.03 & 46 & $(-54,143)$ & 19 & 128 & 278.3 \\
\hline Wake, NC & 6306 & $(6108,6498)$ & 6693 & 1.06 & 387 & $(195,585)$ & 34 & 504 & 130.2 \\
\hline Wayne, NC & 1366 & $(1276,1461)$ & 1543 & 1.13 & 177 & $(82,267)$ & 143 & 165 & 93.2 \\
\hline Wilkes, NC & 907 & $(835,981)$ & 985 & 1.09 & 78 & $(4,150)$ & 114 & 88 & 112.8 \\
\hline Wilson, NC & 958 & $(885,1033)$ & 1126 & 1.18 & 168 & $(93,241)$ & 206 & 129 & 76.8 \\
\hline $\begin{array}{l}\text { Benson and Nearby Coun- } \\
\text { ties, ND }\end{array}$ & 576 & $(518,635)$ & 762 & 1.32 & 186 & $(127,244)$ & 374 & 136 & 73.1 \\
\hline Cass, ND & 1286 & $(1207,1365)$ & 1432 & 1.11 & 146 & $(67,225)$ & 77 & 176 & 120.5 \\
\hline $\begin{array}{l}\text { LaMoure and Nearby } \\
\text { Counties, ND }\end{array}$ & 755 & $(687,816)$ & 913 & 1.21 & 158 & $(97,226)$ & 244 & 185 & 117.1 \\
\hline $\begin{array}{l}\text { McHenry and Nearby } \\
\text { Counties, ND }\end{array}$ & 741 & $(679,808)$ & 1088 & 1.47 & 347 & $(280,409)$ & 379 & 251 & 72.3 \\
\hline Northeast North Dakota, & 986 & $(908,1060)$ & 1064 & 1.08 & 78 & $(4,156)$ & 74 & 143 & 183.3 \\
\hline
\end{tabular}


Table C1: Primary Estimates for Each County-Set

\begin{tabular}{|c|c|c|c|c|c|c|c|c|c|}
\hline County-Set & $\begin{array}{l}\text { Expected } \\
\text { Deaths }\end{array}$ & $\begin{array}{l}\text { Expected } \\
\text { Deaths } 95 \% \text { CI }\end{array}$ & $\begin{array}{l}\text { Observed } \\
\text { Deaths }\end{array}$ & $\begin{array}{l}\text { Observed/ } \\
\text { Expected }\end{array}$ & $\begin{array}{l}\text { Excess } \\
\text { Deaths }\end{array}$ & $\begin{array}{l}\text { Excess Deaths } \\
95 \% \mathrm{CI}\end{array}$ & $\begin{array}{l}\text { Excess Deaths } \\
\text { Per } 100,000\end{array}$ & $\begin{array}{l}\text { COVID-19 } \\
\text { Deaths }\end{array}$ & $\begin{array}{c}\text { COVID-19 to } \\
\text { Excess Ratio (\%) }\end{array}$ \\
\hline $\begin{array}{l}\text { Northwest North Dakota, } \\
\text { ND }\end{array}$ & 708 & $(648,772)$ & 883 & 1.25 & 175 & $(111,235)$ & 160 & 129 & 73.7 \\
\hline $\begin{array}{l}\text { South Central North } \\
\text { Dakota, ND }\end{array}$ & 878 & $(808,952)$ & 1202 & 1.37 & 324 & $(250,394)$ & 307 & 212 & 65.4 \\
\hline $\begin{array}{l}\text { Southwest North Dakota, } \\
\text { ND }\end{array}$ & 566 & $(510,625)$ & 710 & 1.25 & 144 & $(85,200)$ & 257 & 105 & 72.9 \\
\hline Adams-Brown, $\mathrm{OH}$ & 969 & $(894,1046)$ & 1061 & 1.09 & 92 & $(15,167)$ & 129 & 66 & 71.7 \\
\hline Allen, $\mathrm{OH}$ & 1200 & $(1115,1287)$ & 1373 & 1.14 & 173 & $(86,258)$ & 169 & 189 & 109.2 \\
\hline Ashland, $\mathrm{OH}$ & 617 & $(561,673)$ & 684 & 1.11 & 67 & $(11,123)$ & 125 & 74 & 110.4 \\
\hline Ashtabula, OH & 1265 & $(1167,1353)$ & 1312 & 1.04 & 47 & $(-41,145)$ & 48 & 144 & 306.4 \\
\hline Athens-Hocking, $\mathrm{OH}$ & 840 & $(772,905)$ & 980 & 1.17 & 140 & $(75,208)$ & 150 & 75 & 53.6 \\
\hline Auglaize-Mercer, $\mathrm{OH}$ & 911 & $(841,988)$ & 1173 & 1.29 & 262 & $(185,332)$ & 302 & 164 & 62.6 \\
\hline Belmont, $\mathrm{OH}$ & 882 & $(812,956)$ & 1018 & 1.15 & 136 & $(62,206)$ & 205 & 104 & 76.5 \\
\hline Butler, OH & 3703 & $(3540,3865)$ & 4218 & 1.14 & 515 & $(353,678)$ & 134 & 439 & 85.2 \\
\hline $\begin{array}{l}\text { Carroll-Harrison- } \\
\text { Tuscarawas, OH }\end{array}$ & 1725 & $(1615,1831)$ & 1972 & 1.14 & 247 & $(141,357)$ & 186 & 241 & 97.6 \\
\hline $\begin{array}{l}\text { Champaign-Logan- } \\
\text { Madison-Shelby, OH }\end{array}$ & 1955 & $(1853,2073)$ & 2183 & 1.12 & 228 & $(110,330)$ & 128 & 200 & 87.7 \\
\hline Clark, OH & 1842 & $(1737,1950)$ & 2083 & 1.13 & 241 & $(133,346)$ & 180 & 233 & 96.7 \\
\hline Clermont, $\mathrm{OH}$ & 2027 & $(1912,2146)$ & 2230 & 1.10 & 203 & $(84,318)$ & 97 & 156 & 76.8 \\
\hline $\begin{array}{l}\text { Clinton-Fayette-Highland, } \\
\mathrm{OH}\end{array}$ & 1389 & $(1301,1475)$ & 1528 & 1.10 & 139 & $(53,227)$ & 121 & 138 & 99.3 \\
\hline Columbiana, $\mathrm{OH}$ & 1391 & $(1290,1487)$ & 1521 & 1.09 & 130 & $(34,231)$ & 128 & 173 & 133.1 \\
\hline Coshocton-Holmes, $\mathrm{OH}$ & 800 & $(726,872)$ & 926 & 1.16 & 126 & $(54,200)$ & 156 & 123 & 97.6 \\
\hline Crawford-Richland, $\mathrm{OH}$ & 2198 & $(2071,2321)$ & 2410 & 1.10 & 212 & $(89,339)$ & 131 & 244 & 115.1 \\
\hline Cuyahoga, OH & 14174 & $(13738,14595)$ & 16198 & 1.14 & 2024 & $(1603,2460)$ & 164 & 1650 & 81.5 \\
\hline Darke-Preble, $\mathrm{OH}$ & 1178 & $(1095,1263)$ & 1269 & 1.08 & 91 & $(6,174)$ & 99 & 185 & 203.3 \\
\hline Defiance-Williams, OH & 914 & $(841,989)$ & 920 & 1.01 & 6 & $(-69,79)$ & 8 & 130 & 2166.7 \\
\hline Delaware, $\mathrm{OH}$ & 1296 & $(1210,1385)$ & 1474 & 1.14 & 178 & $(89,264)$ & 83 & 99 & 55.6 \\
\hline Erie, $\mathrm{OH}$ & 1017 & $(942,1094)$ & 1183 & 1.16 & 166 & $(89,241)$ & 224 & 135 & 81.3 \\
\hline Fairfield, OH & 1540 & $(1448,1634)$ & 1688 & 1.10 & 148 & $(54,240)$ & 93 & 154 & 104.1 \\
\hline Franklin, $\mathrm{OH}$ & 10589 & $(10278,10912)$ & 11873 & 1.12 & 1284 & $(961,1595)$ & 96 & 1197 & 93.2 \\
\hline Fulton-Henry, OH & 787 & $(719,852)$ & 883 & 1.12 & 96 & $(31,164)$ & 138 & 111 & 115.6 \\
\hline Gallia-Meigs, OH & 771 & $(706,836)$ & 808 & 1.05 & 37 & $(-28,102)$ & 70 & 61 & 164.9 \\
\hline Geauga, $\mathrm{OH}$ & 897 & $(825,972)$ & 1031 & 1.15 & 134 & $(59,206)$ & 142 & 129 & 96.3 \\
\hline Greene, $\mathrm{OH}$ & 1675 & $(1580,1773)$ & 1875 & 1.12 & 200 & $(102,295)$ & 117 & 183 & 91.5 \\
\hline $\begin{array}{l}\text { Guernsey-Monroe-Noble, } \\
\text { OH }\end{array}$ & 850 & $(780,921)$ & 896 & 1.05 & 46 & $(-25,116)$ & 69 & 104 & 226.1 \\
\hline Hamilton, $\mathrm{OH}$ & 8330 & $(8072,8595)$ & 9353 & 1.12 & 1023 & $(758,1281)$ & 125 & 871 & 85.1 \\
\hline Hancock, OH & 851 & $(783,923)$ & 915 & 1.08 & 64 & $(-8,132)$ & 84 & 110 & 171.9 \\
\hline Hardin-Wyandot, $\mathrm{OH}$ & 614 & $(553,674)$ & 714 & 1.16 & 100 & $(40,161)$ & 186 & 98 & 98.0 \\
\hline Huron, $\mathrm{OH}$ & 650 & $(589,708)$ & 788 & 1.21 & 138 & $(80,199)$ & 236 & 88 & 63.8 \\
\hline Jackson-Pike-Vinton, OH & 1034 & $(957,1114)$ & 1116 & 1.08 & 82 & $(2,159)$ & 112 & 70 & 85.4 \\
\hline Jefferson, $\mathrm{OH}$ & 918 & $(834,998)$ & 1010 & 1.10 & 92 & $(12,176)$ & 143 & 99 & 107.6 \\
\hline Knox-Morrow, OH & 985 & $(910,1056)$ & 1148 & 1.17 & 163 & $(92,238)$ & 166 & 110 & 67.5 \\
\hline Lake, $\mathrm{OH}$ & 2674 & $(2535,2811)$ & 2932 & 1.10 & 258 & $(121,397)$ & 112 & 279 & 108.1 \\
\hline Lawrence, $\mathrm{OH}$ & 940 & $(867,1022)$ & 960 & 1.02 & 20 & $(-62,93)$ & 33 & 79 & 395.0 \\
\hline Licking, $\mathrm{OH}$ & 1847 & $(1734,1953)$ & 1953 & 1.06 & 106 & $(0,219)$ & 59 & 169 & 159.4 \\
\hline Lorain, $\mathrm{OH}$ & 3412 & $(3261,3574)$ & 3673 & 1.08 & 261 & $(99,412)$ & 83 & 356 & 136.4 \\
\hline Lucas-Ottawa, OH & 5388 & $(5184,5593)$ & 6275 & 1.16 & 887 & $(682,1091)$ & 189 & 709 & 79.9 \\
\hline Mahoning, OH & 3088 & $(2930,3245)$ & 3804 & 1.23 & 716 & $(559,874)$ & 315 & 512 & 71.5 \\
\hline Marion, $\mathrm{OH}$ & 857 & $(783,928)$ & 842 & 0.98 & -15 & $(-86,59)$ & -22 & 121 & NA \\
\hline Medina, $\mathrm{OH}$ & 1654 & $(1559,1748)$ & 1865 & 1.13 & 211 & $(117,306)$ & 117 & 195 & 92.4 \\
\hline Miami, OH & 1212 & $(1126,1301)$ & 1310 & 1.08 & 98 & $(9,184)$ & 90 & 138 & 140.8 \\
\hline Montgomery, $\mathrm{OH}$ & 6750 & $(6498,7007)$ & 7478 & 1.11 & 728 & $(471,980)$ & 137 & 764 & 104.9 \\
\hline Morgan-Perry, OH & 567 & $(515,625)$ & 667 & 1.18 & 100 & $(42,152)$ & 198 & 56 & 56.0 \\
\hline
\end{tabular}


Table C1: Primary Estimates for Each County-Set

\begin{tabular}{|c|c|c|c|c|c|c|c|c|c|}
\hline County-Set & $\begin{array}{l}\text { Expected } \\
\text { Deaths }\end{array}$ & $\begin{array}{l}\text { Expected } \\
\text { Deaths } 95 \% \mathrm{CI}\end{array}$ & $\begin{array}{l}\text { Observed } \\
\text { Deaths }\end{array}$ & $\begin{array}{l}\text { Observed/ } \\
\text { Expected }\end{array}$ & $\begin{array}{l}\text { Excess } \\
\text { Deaths }\end{array}$ & $\begin{array}{l}\text { Excess Deaths } \\
95 \% \text { CI }\end{array}$ & $\begin{array}{l}\text { Excess Deaths } \\
\text { Per } 100,000\end{array}$ & $\begin{array}{l}\text { COVID-19 } \\
\text { Deaths }\end{array}$ & $\begin{array}{l}\text { COVID-19 to } \\
\text { Excess Ratio (\%) }\end{array}$ \\
\hline Muskingum, $\mathrm{OH}$ & 1076 & $(996,1161)$ & 1156 & 1.07 & 80 & $(-5,160)$ & 93 & 96 & 120.0 \\
\hline $\begin{array}{l}\text { Paulding-Putnam-Van } \\
\text { Wert, OH }\end{array}$ & 880 & $(811,955)$ & 1042 & 1.18 & 162 & $(87,231)$ & 201 & 181 & 111.7 \\
\hline Pickaway, OH & 685 & $(624,750)$ & 719 & 1.05 & 34 & $(-31,95)$ & 57 & 98 & 288.2 \\
\hline Portage, $\mathrm{OH}$ & 1635 & $(1536,1740)$ & 1699 & 1.04 & 64 & $(-41,163)$ & 39 & 133 & 207.8 \\
\hline Ross, OH & 918 & $(847,991)$ & 1095 & 1.19 & 177 & $(104,248)$ & 230 & 116 & 65.5 \\
\hline Sandusky, OH & 737 & $(668,795)$ & 745 & 1.01 & 8 & $(-50,77)$ & 15 & 93 & 1162.5 \\
\hline Scioto, $\mathrm{OH}$ & 1145 & $(1056,1232)$ & 1305 & 1.14 & 160 & $(73,249)$ & 214 & 79 & 49.4 \\
\hline Seneca, OH & 638 & $(581,698)$ & 722 & 1.13 & 84 & $(24,141)$ & 152 & 83 & 98.8 \\
\hline Stark, OH & 4619 & $(4428,4814)$ & 5286 & 1.14 & 667 & $(472,858)$ & 180 & 690 & 103.4 \\
\hline Summit, OH & 6145 & $(5907,6367)$ & 6860 & 1.12 & 715 & $(493,953)$ & 132 & 703 & 98.3 \\
\hline Trumbull, OH & 2700 & $(2553,2843)$ & 3133 & 1.16 & 433 & $(290,580)$ & 220 & 376 & 86.8 \\
\hline Union, $\mathrm{OH}$ & 452 & $(402,502)$ & 488 & 1.08 & 36 & $(-14,86)$ & 60 & 34 & 94.4 \\
\hline Warren, $\mathrm{OH}$ & 2006 & $(1895,2119)$ & 2228 & 1.11 & 222 & $(109,333)$ & 93 & 225 & 101.4 \\
\hline Washington, $\mathrm{OH}$ & 784 & $(714,856)$ & 835 & 1.07 & 51 & $(-21,121)$ & 86 & 83 & 162.7 \\
\hline Wayne, OH & 1247 & $(1158,1339)$ & 1378 & 1.11 & 131 & $(39,220)$ & 114 & 175 & 133.6 \\
\hline Wood, OH & 1285 & $(1199,1368)$ & 1393 & 1.08 & 108 & $(25,194)$ & 82 & 161 & 149.1 \\
\hline $\begin{array}{l}\text { Adair-Cherokee-Sequoyah, } \\
\text { OK }\end{array}$ & 1329 & $(1235,1416)$ & 1540 & 1.16 & 211 & $(124,305)$ & 190 & 156 & 73.9 \\
\hline Atoka-Bryan-Coal, OK & 827 & $(757,899)$ & 939 & 1.14 & 112 & $(40,182)$ & 165 & 105 & 93.8 \\
\hline $\begin{array}{l}\text { Beckham-Custer-Roger } \\
\text { Mills-Washita, OK }\end{array}$ & 756 & $(690,823)$ & 923 & 1.22 & 167 & $(100,233)$ & 255 & 169 & 101.2 \\
\hline Caddo-Grady, OK & 973 & $(901,1051)$ & 1181 & 1.21 & 208 & $(130,280)$ & 244 & 191 & 91.8 \\
\hline Canadian, OK & 1169 & $(1086,1258)$ & 1293 & 1.11 & 124 & $(35,207)$ & 81 & 175 & 141.1 \\
\hline $\begin{array}{l}\text { Carter-Johnston-Love- } \\
\text { Marshall, OK }\end{array}$ & 1061 & $(982,1141)$ & 1232 & 1.16 & 171 & $(91,250)$ & 198 & 117 & 68.4 \\
\hline $\begin{array}{l}\text { Choctaw-McCurtain- } \\
\text { Pushmataha, OK }\end{array}$ & 800 & $(731,869)$ & 995 & 1.24 & 195 & $(126,264)$ & 336 & 134 & 68.7 \\
\hline Cleveland, OK & 2143 & $(2037,2259)$ & 2439 & 1.14 & 296 & $(180,402)$ & 104 & 285 & 96.3 \\
\hline Comanche, OK & 1094 & $(1021,1179)$ & 1279 & 1.17 & 185 & $(100,258)$ & 157 & 107 & 57.8 \\
\hline $\begin{array}{l}\text { Cotton-Jefferson-Stephens, } \\
\text { OK }\end{array}$ & 758 & $(690,828)$ & 825 & 1.09 & 67 & $(-3,135)$ & 123 & 87 & 129.9 \\
\hline Craig-Mayes, OK & 713 & $(645,776)$ & 778 & 1.09 & 65 & $(2,133)$ & 118 & 85 & 130.8 \\
\hline Creek, OK & 890 & $(816,962)$ & 977 & 1.10 & 87 & $(15,161)$ & 122 & 114 & 131.0 \\
\hline Delaware-Ottawa, OK & 1027 & $(944,1105)$ & 1183 & 1.15 & 156 & $(78,239)$ & 212 & 166 & 106.4 \\
\hline Garfield, OK & 684 & $(622,750)$ & 838 & 1.23 & 154 & $(88,216)$ & 257 & 134 & 87.0 \\
\hline $\begin{array}{l}\text { Garvin-McClain-Murray, } \\
\text { OK }\end{array}$ & 963 & $(885,1044)$ & 1086 & 1.13 & 123 & $(42,201)$ & 148 & 144 & 117.1 \\
\hline Grant-Kay, OK & 672 & $(607,731)$ & 786 & 1.17 & 114 & $(55,179)$ & 239 & 114 & 100.0 \\
\hline $\begin{array}{l}\text { Haskell-Latimer-Pittsburg, } \\
\text { OK }\end{array}$ & 972 & $(901,1055)$ & 1005 & 1.03 & 33 & $(-50,104)$ & 49 & 89 & 269.7 \\
\hline $\begin{array}{l}\text { Hughes-Okfuskee- } \\
\text { Pontotoc-Seminole, OK }\end{array}$ & 1208 & $(1121,1302)$ & 1364 & 1.13 & 156 & $(62,243)$ & 178 & 153 & 98.1 \\
\hline Le Flore, OK & 667 & $(605,727)$ & 743 & 1.11 & 76 & $(16,138)$ & 152 & 67 & 88.2 \\
\hline Lincoln-Logan, OK & 809 & $(743,874)$ & 879 & 1.09 & 70 & $(5,136)$ & 84 & 93 & 132.9 \\
\hline McIntosh-Okmulgee, OK & 838 & $(772,914)$ & 983 & 1.17 & 145 & $(69,211)$ & 253 & 112 & 77.2 \\
\hline $\begin{array}{l}\text { Major and Nearby Coun- } \\
\text { ties, OK }\end{array}$ & 633 & $(573,695)$ & 724 & 1.14 & 91 & $(29,151)$ & 174 & 112 & 123.1 \\
\hline Muskogee, OK & 930 & $(858,1009)$ & 1064 & 1.14 & 134 & $(55,206)$ & 199 & 119 & 88.8 \\
\hline Noble-Osage-Pawnee, OK & 843 & $(777,916)$ & 920 & 1.09 & 77 & $(4,143)$ & 105 & 95 & 123.4 \\
\hline Nowata-Washington, OK & 830 & $(760,898)$ & 853 & 1.03 & 23 & $(-45,93)$ & 37 & 94 & 408.7 \\
\hline Oklahoma (county), OK & 7511 & $(7256,7765)$ & 8934 & 1.19 & 1423 & $(1169,1678)$ & 177 & 1020 & 71.7 \\
\hline Oklahoma Panhandle, OK & 580 & $(522,638)$ & 647 & 1.12 & 67 & $(9,125)$ & 123 & 96 & 143.3 \\
\hline Payne, OK & 628 & $(571,688)$ & 686 & 1.09 & 58 & $(-2,115)$ & 71 & 58 & 100.0 \\
\hline Pottawatomie, OK & 921 & $(851,997)$ & 1009 & 1.10 & 88 & $(12,158)$ & 120 & 87 & 98.9 \\
\hline Rogers, OK & 991 & $(910,1063)$ & 1098 & 1.11 & 107 & $(35,188)$ & 116 & 122 & 114.0 \\
\hline
\end{tabular}


Table C1: Primary Estimates for Each County-Set

\begin{tabular}{|c|c|c|c|c|c|c|c|c|c|}
\hline County-Set & $\begin{array}{l}\text { Expected } \\
\text { Deaths }\end{array}$ & $\begin{array}{l}\text { Expected } \\
\text { Deaths } 95 \% \text { CI }\end{array}$ & $\begin{array}{l}\text { Observed } \\
\text { Deaths }\end{array}$ & $\begin{array}{l}\text { Observed/ } \\
\text { Expected }\end{array}$ & $\begin{array}{l}\text { Excess } \\
\text { Deaths }\end{array}$ & $\begin{array}{l}\text { Excess Deaths } \\
95 \% \text { CI }\end{array}$ & $\begin{array}{l}\text { Excess Deaths } \\
\text { Per } 100,000\end{array}$ & $\begin{array}{l}\text { COVID-19 } \\
\text { Deaths }\end{array}$ & $\begin{array}{l}\text { COVID-19 to } \\
\text { Excess Ratio (\%) }\end{array}$ \\
\hline Southwest Oklahoma, OK & 600 & $(540,658)$ & 762 & 1.27 & 162 & $(104,222)$ & 334 & 135 & 83.3 \\
\hline Tulsa, OK & 6113 & $(5902,6314)$ & 7046 & 1.15 & 933 & $(732,1144)$ & 143 & 735 & 78.8 \\
\hline Wagoner, OK & 668 & $(612,729)$ & 784 & 1.17 & 116 & $(55,172)$ & 141 & 78 & 67.2 \\
\hline $\begin{array}{l}\text { Baker-Harney-Lake- } \\
\text { Malheur, OR }\end{array}$ & 734 & $(675,806)$ & 837 & 1.14 & 103 & $(31,162)$ & 165 & 67 & 65.0 \\
\hline Benton, OR & 599 & $(543,655)$ & 616 & 1.03 & 17 & $(-39,73)$ & 18 & 13 & 76.5 \\
\hline Clackamas, OR & 3608 & $(3458,3764)$ & 3768 & 1.04 & 160 & $(4,310)$ & 38 & 137 & 85.6 \\
\hline $\begin{array}{l}\text { Clatsop-Lincoln- } \\
\text { Tillamook, OR }\end{array}$ & 1406 & $(1317,1497)$ & 1443 & 1.03 & 37 & $(-54,126)$ & 31 & 27 & 73.0 \\
\hline Columbia-Washington, OR & 4140 & $(3976,4300)$ & 4316 & 1.04 & 176 & $(16,340)$ & 26 & 170 & 96.6 \\
\hline Coos-Curry, OR & 1384 & $(1286,1483)$ & 1410 & 1.02 & 26 & $(-73,124)$ & 29 & 17 & 65.4 \\
\hline Deschutes, OR & 1630 & $(1533,1725)$ & 1589 & 0.97 & -41 & $(-136,56)$ & -20 & 27 & NA \\
\hline Douglas, OR & 1614 & $(1511,1721)$ & 1689 & 1.05 & 75 & $(-32,178)$ & 67 & 38 & 50.7 \\
\hline Hood River-Jefferson- & 776 & $(710,841)$ & 860 & 1.11 & 84 & $(19,150)$ & 109 & 65 & 77.4 \\
\hline Sherman-Wasco, OR & & & & & & & & & \\
\hline Jackson, OR & 2535 & $(2402,2669)$ & 2641 & 1.04 & 106 & $(-28,239)$ & 47 & 76 & 71.7 \\
\hline Josephine, OR & 1273 & $(1183,1361)$ & 1334 & 1.05 & 61 & $(-27,151)$ & 69 & 26 & 42.6 \\
\hline Klamath, OR & 907 & $(835,975)$ & 923 & 1.02 & 16 & $(-52,88)$ & 23 & 26 & 162.5 \\
\hline Lane, OR & 3898 & $(3736,4068)$ & 4053 & 1.04 & 155 & $(-15,317)$ & 40 & 89 & 57.4 \\
\hline Linn, OR & 1460 & $(1368,1557)$ & 1495 & 1.02 & 35 & $(-62,127)$ & 26 & 42 & 120.0 \\
\hline Marion, OR & 3021 & $(2888,3161)$ & 3174 & 1.05 & 153 & $(13,286)$ & 43 & 214 & 139.9 \\
\hline Multnomah, OR & 5861 & $(5653,6063)$ & 6473 & 1.10 & 612 & $(410,820)$ & 75 & 433 & 70.8 \\
\hline Polk, OR & 759 & $(693,823)$ & 784 & 1.03 & 25 & $(-39,91)$ & 28 & 34 & 136.0 \\
\hline $\begin{array}{l}\text { Umatilla and Nearby } \\
\text { Counties, OR }\end{array}$ & 1614 & $(1516,1714)$ & 1722 & 1.07 & 108 & $(8,206)$ & 67 & 111 & 102.8 \\
\hline Yamhill, OR & 1041 & $(966,1125)$ & 1049 & 1.01 & 8 & $(-76,83)$ & 7 & 41 & 512.5 \\
\hline Adams, PA & 1127 & $(1052,1210)$ & 1182 & 1.05 & 55 & $(-28,130)$ & 53 & 100 & 181.8 \\
\hline Allegheny, PA & 13910 & $(13491,14325)$ & 15201 & 1.09 & 1291 & $(876,1710)$ & 106 & 1237 & 95.8 \\
\hline Armstrong, PA & 880 & $(805,952)$ & 938 & 1.07 & 58 & $(-14,133)$ & 90 & 77 & 132.8 \\
\hline Beaver, PA & 2247 & $(2126,2367)$ & 2462 & 1.10 & 215 & $(95,336)$ & 132 & 278 & 129.3 \\
\hline Bedford-Fulton, PA & 788 & $(724,864)$ & 923 & 1.17 & 135 & $(59,199)$ & 216 & 123 & 91.1 \\
\hline Berks, PA & 4182 & $(4004,4354)$ & 4624 & 1.11 & 442 & $(270,620)$ & 104 & 614 & 138.9 \\
\hline Blair, PA & 1631 & $(1530,1737)$ & 1906 & 1.17 & 275 & $(169,376)$ & 227 & 203 & 73.8 \\
\hline Bradford-Sullivan, PA & 821 & $(748,896)$ & 823 & 1.00 & 2 & $(-73,75)$ & 2 & 67 & 3350.0 \\
\hline Bucks, PA & 6517 & $(6286,6734)$ & 7218 & 1.11 & 701 & $(484,932)$ & 111 & 965 & 137.7 \\
\hline Butler, PA & 2135 & $(2017,2259)$ & 2327 & 1.09 & 192 & $(68,310)$ & 101 & 255 & 132.8 \\
\hline Cambria, PA & 1937 & $(1817,2061)$ & 2165 & 1.12 & 228 & $(104,348)$ & 177 & 315 & 138.2 \\
\hline Carbon, PA & 828 & $(760,897)$ & 972 & 1.17 & 144 & $(75,212)$ & 222 & 95 & 66.0 \\
\hline Centre, PA & 1014 & $(938,1087)$ & 1224 & 1.21 & 210 & $(137,286)$ & 128 & 158 & 75.2 \\
\hline Chester, PA & 4231 & $(4066,4403)$ & 4664 & 1.10 & 433 & $(261,598)$ & 82 & 602 & 139.0 \\
\hline Clarion-Jefferson, PA & 991 & $(916,1065)$ & 1145 & 1.16 & 154 & $(80,229)$ & 188 & 119 & 77.3 \\
\hline Clearfield, PA & 992 & $(916,1072)$ & 1016 & 1.02 & 24 & $(-56,100)$ & 30 & 60 & 250.0 \\
\hline Clinton-Potter-Tioga, PA & 1150 & $(1067,1231)$ & 1170 & 1.02 & 20 & $(-61,103)$ & 21 & 130 & 650.0 \\
\hline Columbia, PA & 733 & $(671,796)$ & 774 & 1.06 & 41 & $(-22,103)$ & 63 & 85 & 207.3 \\
\hline Crawford, PA & 1015 & $(939,1091)$ & 1094 & 1.08 & 79 & $(3,155)$ & 94 & 105 & 132.9 \\
\hline Cumberland-Perry, PA & 3000 & $(2859,3151)$ & 3324 & 1.11 & 324 & $(173,465)$ & 107 & 383 & 118.2 \\
\hline Dauphin, PA & 2627 & $(2500,2760)$ & 3030 & 1.15 & 403 & $(270,530)$ & 144 & 370 & 91.8 \\
\hline Delaware, PA & 5573 & $(5358,5775)$ & 6586 & 1.18 & 1013 & $(811,1228)$ & 179 & 1042 & 102.9 \\
\hline Erie, PA & 2980 & $(2850,3123)$ & 3296 & 1.11 & 316 & $(173,446)$ & 118 & 277 & 87.7 \\
\hline Fayette-Greene, PA & 2307 & $(2185,2427)$ & 2477 & 1.07 & 170 & $(50,292)$ & 103 & 220 & 129.4 \\
\hline Franklin, PA & 1711 & $(1605,1810)$ & 1980 & 1.16 & 269 & $(170,375)$ & 173 & 258 & 95.9 \\
\hline $\begin{array}{l}\text { Huntingdon-Juniata- } \\
\text { Mifflin, PA }\end{array}$ & 1370 & $(1281,1461)$ & 1644 & 1.20 & 274 & $(183,363)$ & 237 & 291 & 106.2 \\
\hline Indiana, PA & 945 & $(875,1016)$ & 1092 & 1.16 & 147 & $(76,217)$ & 175 & 131 & 89.1 \\
\hline Lackawanna-Wyoming, PA & 3070 & $(2927,3212)$ & 3284 & 1.07 & 214 & $(72,357)$ & 90 & 321 & 150.0 \\
\hline Lancaster, PA & 5366 & $(5167,5552)$ & 6070 & 1.13 & 704 & $(518,903)$ & 128 & 793 & 112.6 \\
\hline
\end{tabular}


Table C1: Primary Estimates for Each County-Set

\begin{tabular}{|c|c|c|c|c|c|c|c|c|c|}
\hline County-Set & $\begin{array}{l}\text { Expected } \\
\text { Deaths }\end{array}$ & $\begin{array}{l}\text { Expected } \\
\text { Deaths } 95 \% \mathrm{CI}\end{array}$ & $\begin{array}{l}\text { Observed } \\
\text { Deaths }\end{array}$ & $\begin{array}{l}\text { Observed/ } \\
\text { Expected }\end{array}$ & $\begin{array}{l}\text { Excess } \\
\text { Deaths }\end{array}$ & $\begin{array}{l}\text { Excess Deaths } \\
95 \% \text { CI }\end{array}$ & $\begin{array}{l}\text { Excess Deaths } \\
\text { Per } 100,000\end{array}$ & $\begin{array}{l}\text { COVID-19 } \\
\text { Deaths }\end{array}$ & $\begin{array}{c}\text { COVID-19 to } \\
\text { Excess Ratio (\%) }\end{array}$ \\
\hline Lawrence, PA & 1228 & $(1142,1322)$ & 1414 & 1.15 & 186 & $(92,272)$ & 218 & 154 & 82.8 \\
\hline Lebanon, PA & 1611 & $(1506,1711)$ & 1809 & 1.12 & 198 & $(98,303)$ & 137 & 165 & 83.3 \\
\hline Lehigh, PA & 3506 & $(3349,3650)$ & 3967 & 1.13 & 461 & $(317,618)$ & 124 & 550 & 119.3 \\
\hline Luzerne, PA & 4203 & $(4034,4394)$ & 4751 & 1.13 & 548 & $(357,717)$ & 173 & 509 & 92.9 \\
\hline Lycoming, PA & 1332 & $(1247,1423)$ & 1478 & 1.11 & 146 & $(55,231)$ & 129 & 154 & 105.5 \\
\hline $\begin{array}{l}\text { McKean and Nearby Coun- } \\
\text { ties, PA }\end{array}$ & 1582 & $(1474,1686)$ & 1652 & 1.04 & 70 & $(-34,178)$ & 58 & 132 & 188.6 \\
\hline Mercer, PA & 1554 & $(1455,1652)$ & 1718 & 1.11 & 164 & $(66,263)$ & 150 & 193 & 117.7 \\
\hline Monroe, PA & 1658 & $(1565,1759)$ & 1811 & 1.09 & 153 & $(52,246)$ & 89 & 230 & 150.3 \\
\hline Montgomery, PA & 7948 & $(7691,8202)$ & 8965 & 1.13 & 1017 & $(763,1274)$ & 122 & 1246 & 122.5 \\
\hline $\begin{array}{l}\text { Montour-Northumberland, } \\
\text { PA }\end{array}$ & 1416 & $(1331,1518)$ & 1726 & 1.22 & 310 & $(208,395)$ & 288 & 280 & 90.3 \\
\hline Northampton, PA & 3247 & $(3100,3389)$ & 3602 & 1.11 & 355 & $(213,502)$ & 115 & 477 & 134.4 \\
\hline Philadelphia, PA & 14211 & $(13824,14598)$ & 18135 & 1.28 & 3924 & $(3537,4311)$ & 247 & 2679 & 68.3 \\
\hline Pike, PA & 599 & $(539,659)$ & 628 & 1.05 & 29 & $(-31,89)$ & 51 & 51 & 175.9 \\
\hline Schuylkill, PA & 1969 & $(1857,2086)$ & 2285 & 1.16 & 316 & $(199,428)$ & 224 & 265 & 83.9 \\
\hline Snyder-Union, PA & 750 & $(685,811)$ & 907 & 1.21 & 157 & $(96,222)$ & 184 & 97 & 61.8 \\
\hline Somerset, PA & 1006 & $(925,1079)$ & 1117 & 1.11 & 111 & $(38,192)$ & 152 & 116 & 104.5 \\
\hline Susquehanna-Wayne, PA & 1153 & $(1074,1240)$ & 1257 & 1.09 & 104 & $(17,183)$ & 114 & 74 & 71.2 \\
\hline Venango, PA & 632 & $(574,693)$ & 754 & 1.19 & 122 & $(61,180)$ & 244 & 58 & 47.5 \\
\hline Washington, PA & 2634 & $(2490,2768)$ & 2790 & 1.06 & 156 & $(22,300)$ & 75 & 157 & 100.6 \\
\hline Westmoreland, PA & 4715 & $(4494,4912)$ & 5078 & 1.08 & 363 & $(166,584)$ & 105 & 486 & 133.9 \\
\hline York, PA & 4480 & $(4305,4652)$ & 4990 & 1.11 & 510 & $(338,685)$ & 113 & 498 & 97.6 \\
\hline Bristol-Providence, RI & 6400 & $(6168,6618)$ & 7563 & 1.18 & 1163 & $(945,1395)$ & 169 & 1465 & 126.0 \\
\hline Kent, RI & 1816 & $(1719,1923)$ & 2009 & 1.11 & 193 & $(86,290)$ & 117 & 256 & 132.6 \\
\hline Newport, RI & 836 & $(768,906)$ & 837 & 1.00 & 1 & $(-69,69)$ & 1 & 41 & 4100.0 \\
\hline Washington, RI & 1289 & $(1202,1377)$ & 1410 & 1.09 & 121 & $(33,208)$ & 96 & 107 & 88.4 \\
\hline $\begin{array}{l}\text { Abbeville-Edgefield- } \\
\text { Greenwood-McCormick, } \\
\text { SC }\end{array}$ & 1646 & $(1543,1752)$ & 1849 & 1.12 & 203 & $(97,306)$ & 153 & 180 & 88.7 \\
\hline Aiken, SC & 1963 & $(1849,2067)$ & 2230 & 1.14 & 267 & $(163,381)$ & 155 & 174 & 65.2 \\
\hline Anderson, SC & 2399 & $(2273,2524)$ & 2868 & 1.20 & 469 & $(344,595)$ & 229 & 315 & 67.2 \\
\hline Beaufort, SC & 1741 & $(1640,1836)$ & 1925 & 1.11 & 184 & $(89,285)$ & 95 & 113 & 61.4 \\
\hline Berkeley, SC & 1760 & $(1661,1859)$ & 1902 & 1.08 & 142 & $(43,241)$ & 60 & 136 & 95.8 \\
\hline Calhoun-Richland, SC & 3448 & $(3303,3601)$ & 4067 & 1.18 & 619 & $(466,764)$ & 142 & 357 & 57.7 \\
\hline Charleston, SC & 3465 & $(3310,3621)$ & 3841 & 1.11 & 376 & $(220,531)$ & 91 & 305 & 81.1 \\
\hline Cherokee, SC & 712 & $(651,776)$ & 814 & 1.14 & 102 & $(38,163)$ & 178 & 88 & 86.3 \\
\hline Chester-Fairfield, SC & 775 & $(708,841)$ & 943 & 1.22 & 168 & $(102,235)$ & 310 & 99 & 58.9 \\
\hline Chesterfield-Marlboro, SC & 934 & $(865,1013)$ & 1117 & 1.20 & 183 & $(104,252)$ & 256 & 107 & 58.5 \\
\hline $\begin{array}{l}\text { Clarendon-Williamsburg, } \\
\text { SC }\end{array}$ & 870 & $(799,943)$ & 1133 & 1.30 & 263 & $(190,334)$ & 416 & 161 & 61.2 \\
\hline Darlington, SC & 911 & $(837,982)$ & 1046 & 1.15 & 135 & $(64,209)$ & 203 & 106 & 78.5 \\
\hline Dillon-Marion, SC & 848 & $(776,920)$ & 1065 & 1.26 & 217 & $(145,289)$ & 354 & 133 & 61.3 \\
\hline Dorchester, SC & 1346 & $(1253,1429)$ & 1469 & 1.09 & 123 & $(40,216)$ & 75 & 116 & 94.3 \\
\hline Florence, SC & 1610 & $(1507,1706)$ & 2022 & 1.26 & 412 & $(316,515)$ & 300 & 256 & 62.1 \\
\hline Georgetown, SC & 854 & $(786,930)$ & 1034 & 1.21 & 180 & $(104,248)$ & 285 & 97 & 53.9 \\
\hline Greenville, SC & 4707 & $(4530,4891)$ & 5330 & 1.13 & 623 & $(439,800)$ & 118 & 497 & 79.8 \\
\hline $\begin{array}{l}\text { Hampton and Nearby } \\
\text { Counties, SC }\end{array}$ & 1751 & $(1648,1851)$ & 2011 & 1.15 & 260 & $(160,363)$ & 199 & 212 & 81.5 \\
\hline Horry, SC & 4218 & $(4039,4378)$ & 4751 & 1.13 & 533 & $(373,712)$ & 145 & 368 & 69.0 \\
\hline Kershaw-Lee, SC & 993 & $(912,1071)$ & 1165 & 1.17 & 172 & $(94,253)$ & 207 & 136 & 79.1 \\
\hline Lancaster, SC & 1000 & $(921,1067)$ & 1161 & 1.16 & 161 & $(94,240)$ & 159 & 113 & 70.2 \\
\hline Laurens, SC & 923 & $(847,1001)$ & 996 & 1.08 & 73 & $(-5,149)$ & 108 & 89 & 121.9 \\
\hline Lexington, SC & 2760 & $(2612,2885)$ & 3097 & 1.12 & 337 & $(212,485)$ & 111 & 262 & 77.7 \\
\hline Newberry-Saluda, SC & 666 & $(604,731)$ & 899 & 1.35 & 233 & $(168,295)$ & 392 & 109 & 46.8 \\
\hline Oconee, SC & 1007 & $(928,1089)$ & 1034 & 1.03 & 27 & $(-55,106)$ & 33 & 84 & 311.1 \\
\hline
\end{tabular}


Table C1: Primary Estimates for Each County-Set

\begin{tabular}{|c|c|c|c|c|c|c|c|c|c|}
\hline County-Set & $\begin{array}{l}\text { Expected } \\
\text { Deaths }\end{array}$ & $\begin{array}{l}\text { Expected } \\
\text { Deaths } 95 \% \text { CI }\end{array}$ & $\begin{array}{l}\text { Observed } \\
\text { Deaths }\end{array}$ & $\begin{array}{l}\text { Observed/ } \\
\text { Expected }\end{array}$ & $\begin{array}{l}\text { Excess } \\
\text { Deaths }\end{array}$ & $\begin{array}{l}\text { Excess Deaths } \\
95 \% \mathrm{CI}\end{array}$ & $\begin{array}{c}\text { Excess Deaths } \\
\text { Per } 100,000\end{array}$ & $\begin{array}{l}\text { COVID-19 } \\
\text { Deaths }\end{array}$ & $\begin{array}{l}\text { COVID-19 to } \\
\text { Excess Ratio (\%) }\end{array}$ \\
\hline Orangeburg, SC & 1137 & $(1059,1227)$ & 1364 & 1.20 & 227 & $(137,305)$ & 265 & 142 & 62.6 \\
\hline Pickens, SC & 1321 & $(1229,1402)$ & 1592 & 1.21 & 271 & $(190,363)$ & 213 & 157 & 57.9 \\
\hline Spartanburg-Union, SC & 3873 & $(3696,4048)$ & 4373 & 1.13 & 500 & $(325,677)$ & 140 & 465 & 93.0 \\
\hline Sumter, SC & 1235 & $(1148,1325)$ & 1435 & 1.16 & 200 & $(110,287)$ & 187 & 138 & 69.0 \\
\hline York, SC & 2349 & $(2230,2476)$ & 2646 & 1.13 & 297 & $(170,416)$ & 102 & 267 & 89.9 \\
\hline $\begin{array}{l}\text { Clark and Nearby Coun- } \\
\text { ties, SD }\end{array}$ & 733 & $(672,797)$ & 940 & 1.28 & 207 & $(143,268)$ & 283 & 185 & 89.4 \\
\hline $\begin{array}{l}\text { Clay-Turner-Union- } \\
\text { Yankton, SD }\end{array}$ & 643 & $(585,708)$ & 687 & 1.07 & 44 & $(-21,102)$ & 70 & 115 & 261.4 \\
\hline $\begin{array}{l}\text { Douglas and Nearby Coun- } \\
\text { ties, SD }\end{array}$ & 675 & $(614,743)$ & 792 & 1.17 & 117 & $(49,178)$ & 217 & 136 & 116.2 \\
\hline Lincoln-Minnehaha, SD & 1901 & $(1790,2006)$ & 2269 & 1.19 & 368 & $(263,479)$ & 140 & 364 & 98.9 \\
\hline $\begin{array}{l}\text { Miner and Nearby Coun- } \\
\text { ties, SD }\end{array}$ & 573 & $(516,627)$ & 642 & 1.12 & 69 & $(15,126)$ & 96 & 119 & 172.5 \\
\hline $\begin{array}{l}\text { North Central South } \\
\text { Dakota, SD }\end{array}$ & 570 & $(512,628)$ & 680 & 1.19 & 110 & $(52,168)$ & 219 & 117 & 106.4 \\
\hline $\begin{array}{l}\text { Northeast South Dakota, } \\
\text { SD }\end{array}$ & 688 & $(631,751)$ & 857 & 1.25 & 169 & $(106,226)$ & 249 & 155 & 91.7 \\
\hline $\begin{array}{l}\text { Northwest South Dakota, } \\
\text { SD }\end{array}$ & 780 & $(715,847)$ & 888 & 1.14 & 108 & $(41,173)$ & 131 & 141 & 130.6 \\
\hline Pennington, SD & 1058 & $(982,1137)$ & 1184 & 1.12 & 126 & $(47,202)$ & 110 & 141 & 111.9 \\
\hline $\begin{array}{l}\text { Southwest Central South } \\
\text { Dakota, SD }\end{array}$ & 616 & $(558,673)$ & 704 & 1.14 & 88 & $(31,146)$ & 186 & 122 & 138.6 \\
\hline Anderson, $\mathrm{TN}$ & 1081 & $(1001,1166)$ & 1112 & 1.03 & 31 & $(-54,111)$ & 40 & 76 & 245.2 \\
\hline $\begin{array}{l}\text { Bedford-Lincoln-Moore, } \\
\text { TN }\end{array}$ & 1098 & $(1014,1183)$ & 1219 & 1.11 & 121 & $(36,205)$ & 132 & 121 & 100.0 \\
\hline Benton-Carroll-Henry, TN & 1230 & $(1141,1324)$ & 1411 & 1.15 & 181 & $(87,270)$ & 235 & 145 & 80.1 \\
\hline $\begin{array}{l}\text { Bledsoe-Rhea-Van Buren- } \\
\text { White, TN }\end{array}$ & 1131 & $(1045,1215)$ & 1196 & 1.06 & 65 & $(-19,151)$ & 80 & 95 & 146.2 \\
\hline Blount-Loudon, TN & 2308 & $(2187,2431)$ & 2422 & 1.05 & 114 & $(-9,235)$ & 60 & 164 & 143.9 \\
\hline Bradley-Polk, TN & 1477 & $(1374,1579)$ & 1671 & 1.13 & 194 & $(92,297)$ & 153 & 92 & 47.4 \\
\hline $\begin{array}{l}\text { Campbell-Claiborne- } \\
\text { Hancock-Scott, TN }\end{array}$ & 1508 & $(1406,1610)$ & 1549 & 1.03 & 41 & $(-61,143)$ & 41 & 104 & 253.7 \\
\hline $\begin{array}{l}\text { Cannon-DeKalb-Warren, } \\
\text { TN }\end{array}$ & 1001 & $(921,1088)$ & 1114 & 1.11 & 113 & $(26,193)$ & 147 & 101 & 89.4 \\
\hline $\begin{array}{l}\text { Carter-Johnson-Unicoi, } \\
\text { TN }\end{array}$ & 1435 & $(1336,1539)$ & 1563 & 1.09 & 128 & $(24,227)$ & 139 & 153 & 119.5 \\
\hline Cheatham-Dickson, TN & 1119 & $(1037,1199)$ & 1134 & 1.01 & 15 & $(-65,97)$ & 15 & 104 & 693.3 \\
\hline Chester-Madison, TN & 1363 & $(1273,1456)$ & 1534 & 1.13 & 171 & $(78,261)$ & 149 & 205 & 119.9 \\
\hline Cocke-Jefferson, TN & 1294 & $(1196,1388)$ & 1421 & 1.10 & 127 & $(33,225)$ & 139 & 137 & 107.9 \\
\hline Coffee-Franklin, TN & 1252 & $(1163,1341)$ & 1444 & 1.15 & 192 & $(103,281)$ & 193 & 142 & 74.0 \\
\hline $\begin{array}{l}\text { Crockett-Gibson- } \\
\text { Haywood, TN }\end{array}$ & 1132 & $(1044,1215)$ & 1381 & 1.22 & 249 & $(166,337)$ & 312 & 172 & 69.1 \\
\hline Cumberland-Fentress, TN & 1196 & $(1109,1276)$ & 1354 & 1.13 & 158 & $(78,245)$ & 199 & 102 & 64.6 \\
\hline Davidson, TN & 5652 & $(5445,5873)$ & 6521 & 1.15 & 869 & $(648,1076)$ & 124 & 589 & 67.8 \\
\hline $\begin{array}{l}\text { Decatur-Henderson-Lewis- } \\
\text { Perry, TN }\end{array}$ & 866 & $(794,940)$ & 1013 & 1.17 & 147 & $(73,219)$ & 243 & 119 & 81.0 \\
\hline Dyer-Lake-Lauderdale, TN & 958 & $(882,1037)$ & 1081 & 1.13 & 123 & $(44,199)$ & 176 & 121 & 98.4 \\
\hline Fayette-Hardeman, TN & 813 & $(748,878)$ & 917 & 1.13 & 104 & $(39,169)$ & 158 & 99 & 95.2 \\
\hline Giles-Marshall, TN & 815 & $(748,887)$ & 933 & 1.14 & 118 & $(46,185)$ & 181 & 80 & 67.8 \\
\hline Grainger-Hamblen, TN & 1151 & $(1062,1231)$ & 1413 & 1.23 & 262 & $(182,351)$ & 295 & 121 & 46.2 \\
\hline Greene, TN & 1043 & $(962,1124)$ & 1130 & 1.08 & 87 & $(6,168)$ & 124 & 92 & 105.7 \\
\hline $\begin{array}{l}\text { Grundy-Marion- } \\
\text { Sequatchie, TN }\end{array}$ & 783 & $(713,848)$ & 852 & 1.09 & 69 & $(4,139)$ & 120 & 69 & 100.0 \\
\hline Hamilton, TN & 3917 & $(3749,4100)$ & 4239 & 1.08 & 322 & $(139,490)$ & 87 & 288 & 89.4 \\
\hline Hardin-McNairy, TN & 842 & $(771,915)$ & 940 & 1.12 & 98 & $(25,169)$ & 190 & 80 & 81.6 \\
\hline
\end{tabular}


Table C1: Primary Estimates for Each County-Set

\begin{tabular}{|c|c|c|c|c|c|c|c|c|c|}
\hline County-Set & $\begin{array}{l}\text { Expected } \\
\text { Deaths }\end{array}$ & $\begin{array}{l}\text { Expected } \\
\text { Deaths } 95 \% \text { CI }\end{array}$ & $\begin{array}{l}\text { Observed } \\
\text { Deaths }\end{array}$ & $\begin{array}{l}\text { Observed/ } \\
\text { Expected }\end{array}$ & $\begin{array}{l}\text { Excess } \\
\text { Deaths }\end{array}$ & $\begin{array}{l}\text { Excess Deaths } \\
95 \% \mathrm{CI}\end{array}$ & $\begin{array}{c}\text { Excess Deaths } \\
\text { Per } 100,000\end{array}$ & $\begin{array}{l}\text { COVID-19 } \\
\text { Deaths }\end{array}$ & $\begin{array}{l}\text { COVID-19 to } \\
\text { Excess Ratio (\%) }\end{array}$ \\
\hline Hawkins, TN & 790 & $(724,859)$ & 903 & 1.14 & 113 & $(44,179)$ & 199 & 61 & 54.0 \\
\hline $\begin{array}{l}\text { Hickman-Houston- } \\
\text { Humphreys-Stewart, } \\
\text { TN }\end{array}$ & 897 & $(820,964)$ & 1017 & 1.13 & 120 & $(53,197)$ & 181 & 92 & 76.7 \\
\hline $\begin{array}{l}\text { Jackson-Putnam-Smith, } \\
\text { TN }\end{array}$ & 1311 & $(1220,1398)$ & 1538 & 1.17 & 227 & $(140,318)$ & 199 & 177 & 78.0 \\
\hline Knox-Union, TN & 5147 & $(4941,5332)$ & 5678 & 1.10 & 531 & $(346,737)$ & 108 & 340 & 64.0 \\
\hline Lawrence-Wayne, TN & 846 & $(776,913)$ & 947 & 1.12 & 101 & $(34,171)$ & 166 & 87 & 86.1 \\
\hline $\begin{array}{l}\text { McMinn-Meigs-Monroe, } \\
\text { TN }\end{array}$ & 1580 & $(1477,1683)$ & 1769 & 1.12 & 189 & $(86,292)$ & 166 & 142 & 75.1 \\
\hline Maury, TN & 1077 & $(999,1154)$ & 1081 & 1.00 & 4 & $(-73,82)$ & 4 & 116 & 2900.0 \\
\hline Montgomery, TN & 1591 & $(1496,1693)$ & 1750 & 1.10 & 159 & $(57,254)$ & 73 & 125 & 78.6 \\
\hline Morgan-Roane, TN & 1105 & $(1018,1187)$ & 1138 & 1.03 & 33 & $(-49,120)$ & 44 & 74 & 224.2 \\
\hline $\begin{array}{l}\text { North Central Tennessee, } \\
\text { TN }\end{array}$ & 932 & $(859,1006)$ & 1133 & 1.22 & 201 & $(127,274)$ & 281 & 151 & 75.1 \\
\hline Obion-Weakley, TN & 810 & $(744,875)$ & 974 & 1.20 & 164 & $(99,230)$ & 258 & 118 & 72.0 \\
\hline Robertson, TN & 740 & $(677,809)$ & 886 & 1.20 & 146 & $(77,209)$ & 200 & 83 & 56.8 \\
\hline Rutherford, TN & 2334 & $(2207,2448)$ & 2649 & 1.13 & 315 & $(201,442)$ & 92 & 250 & 79.4 \\
\hline Sevier, TN & 1204 & $(1125,1290)$ & 1286 & 1.07 & 82 & $(-4,161)$ & 82 & 88 & 107.3 \\
\hline Shelby, TN & 8791 & $(8517,9100)$ & 10654 & 1.21 & 1863 & $(1554,2137)$ & 199 & 1042 & 55.9 \\
\hline Sullivan, TN & 2209 & $(2086,2339)$ & 2531 & 1.15 & 322 & $(192,445)$ & 202 & 205 & 63.7 \\
\hline Sumner, TN & 1817 & $(1715,1919)$ & 2026 & 1.12 & 209 & $(107,311)$ & 108 & 210 & 100.5 \\
\hline Tipton, TN & 685 & $(624,747)$ & 710 & 1.04 & 25 & $(-37,86)$ & 40 & 65 & 260.0 \\
\hline Washington, TN & 1565 & $(1473,1666)$ & 1811 & 1.16 & 246 & $(145,338)$ & 189 & 161 & 65.4 \\
\hline Williamson, TN & 1529 & $(1446,1620)$ & 1627 & 1.06 & 98 & $(7,181)$ & 40 & 144 & 146.9 \\
\hline Wilson, TN & 1269 & $(1182,1356)$ & 1525 & 1.20 & 256 & $(169,343)$ & 172 & 150 & 58.6 \\
\hline Anderson-Houston, TX & 999 & $(921,1083)$ & 1092 & 1.09 & 93 & $(9,171)$ & 115 & 124 & 133.3 \\
\hline Angelina, TX & 948 & $(872,1026)$ & 1162 & 1.23 & 214 & $(136,290)$ & 250 & 173 & 80.8 \\
\hline $\begin{array}{l}\text { Aransas-Calhoun-Refugio, } \\
\text { TX }\end{array}$ & 585 & $(528,645)$ & 801 & 1.37 & 216 & $(156,273)$ & 443 & 56 & 25.9 \\
\hline Archer-Wichita, TX & 1554 & $(1459,1651)$ & 1730 & 1.11 & 176 & $(79,271)$ & 124 & 258 & 146.6 \\
\hline $\begin{array}{l}\text { Atascosa-Live } \\
\text { McMullen, TX }\end{array}$ & 580 & $(523,638)$ & 748 & 1.29 & 168 & $(110,225)$ & 256 & 118 & 70.2 \\
\hline $\begin{array}{l}\text { Austin-Waller- } \\
\text { Washington, TX }\end{array}$ & 1033 & $(949,1110)$ & 1261 & 1.22 & 228 & $(151,312)$ & 186 & 119 & 52.2 \\
\hline Bandera-Kendall-Kerr, TX & 1473 & $(1378,1568)$ & 1599 & 1.09 & 126 & $(31,221)$ & 100 & 114 & 90.5 \\
\hline Bastrop-Caldwell, TX & 1183 & $(1104,1266)$ & 1308 & 1.11 & 125 & $(42,204)$ & 92 & 106 & 84.8 \\
\hline Bee-Goliad-Karnes, TX & 529 & $(476,579)$ & 585 & 1.11 & 56 & $(6,109)$ & 99 & 83 & 148.2 \\
\hline Bell, TX & 2566 & $(2438,2695)$ & 2832 & 1.10 & 266 & $(137,394)$ & 71 & 221 & 83.1 \\
\hline Bexar-Wilson, TX & 14958 & $(14601,15316)$ & 17163 & 1.15 & 2205 & $(1847,2562)$ & 105 & 2121 & 96.2 \\
\hline Bosque-Hamilton-Hill, TX & 920 & $(849,993)$ & 983 & 1.07 & 63 & $(-10,134)$ & 96 & 82 & 130.2 \\
\hline Bowie, TX & 1130 & $(1051,1213)$ & 1243 & 1.10 & 113 & $(30,192)$ & 119 & 137 & 121.2 \\
\hline Brazoria, TX & 2562 & $(2438,2686)$ & 2882 & 1.12 & 320 & $(196,444)$ & 83 & 256 & 80.0 \\
\hline $\begin{array}{l}\text { Brazos-Burleson-Grimes- } \\
\text { Robertson, TX }\end{array}$ & 1905 & $(1813,2008)$ & 2194 & 1.15 & 289 & $(186,381)$ & 97 & 253 & 87.5 \\
\hline Brown-Coleman-Mills, TX & 747 & $(683,814)$ & 901 & 1.21 & 154 & $(87,218)$ & 301 & 120 & 77.9 \\
\hline $\begin{array}{l}\text { Burnet-Lampasas-Llano, } \\
\text { TX }\end{array}$ & 1171 & $(1091,1260)$ & 1257 & 1.07 & 86 & $(-3,166)$ & 92 & 75 & 87.2 \\
\hline $\begin{array}{l}\text { Callahan and Nearby } \\
\text { Counties, TX }\end{array}$ & 762 & $(696,831)$ & 971 & 1.27 & 209 & $(140,275)$ & 322 & 109 & 52.2 \\
\hline Cameron, TX & 2923 & $(2792,3043)$ & 4243 & 1.45 & 1320 & $(1200,1451)$ & 310 & 1136 & 86.1 \\
\hline Cass-Marion-Morris, TX & 781 & $(712,855)$ & 924 & 1.18 & 143 & $(69,212)$ & 273 & 103 & 72.0 \\
\hline Chambers-Liberty, TX & 1240 & $(1156,1330)$ & 1274 & 1.03 & 34 & $(-56,118)$ & 25 & 135 & 397.1 \\
\hline Cherokee, TX & 602 & $(545,660)$ & 687 & 1.14 & 85 & $(27,142)$ & 159 & 102 & 120.0 \\
\hline Clay-Cooke-Montague, TX & 855 & $(781,929)$ & 1013 & 1.18 & 158 & $(84,232)$ & 219 & 103 & 65.2 \\
\hline Collin, TX & 5201 & $(5025,5378)$ & 5721 & 1.10 & 520 & $(343,696)$ & 48 & 483 & 92.9 \\
\hline
\end{tabular}


Table C1: Primary Estimates for Each County-Set

\begin{tabular}{|c|c|c|c|c|c|c|c|c|c|}
\hline County-Set & $\begin{array}{l}\text { Expected } \\
\text { Deaths }\end{array}$ & $\begin{array}{l}\text { Expected } \\
\text { Deaths } 95 \% \text { CI }\end{array}$ & $\begin{array}{l}\text { Observed } \\
\text { Deaths }\end{array}$ & $\begin{array}{l}\text { Observed/ } \\
\text { Expected }\end{array}$ & $\begin{array}{l}\text { Excess } \\
\text { Deaths }\end{array}$ & $\begin{array}{l}\text { Excess Deaths } \\
95 \% \text { CI }\end{array}$ & $\begin{array}{l}\text { Excess Deaths } \\
\text { Per } 100,000\end{array}$ & $\begin{array}{l}\text { COVID-19 } \\
\text { Deaths }\end{array}$ & $\begin{array}{c}\text { COVID-19 to } \\
\text { Excess Ratio (\%) }\end{array}$ \\
\hline Comal, TX & 1433 & $(1340,1530)$ & 1614 & 1.13 & 181 & $(84,274)$ & 110 & 150 & 82.9 \\
\hline $\begin{array}{l}\text { Comanche-Erath- } \\
\text { Somervell, TX }\end{array}$ & 667 & $(606,728)$ & 716 & 1.07 & 49 & $(-12,110)$ & 74 & 90 & 183.7 \\
\hline Coryell, TX & 552 & $(499,609)$ & 612 & 1.11 & 60 & $(3,113)$ & 80 & 44 & 73.3 \\
\hline $\begin{array}{l}\text { Crockett-Kinney-Terrell- } \\
\text { Val Verde, TX }\end{array}$ & 523 & $(472,578)$ & 693 & 1.33 & 170 & $(115,221)$ & 297 & 188 & 110.6 \\
\hline $\begin{array}{l}\text { Crosby-Floyd-Hale- } \\
\text { Swisher, TX }\end{array}$ & 575 & $(522,632)$ & 778 & 1.35 & 203 & $(146,256)$ & 390 & 204 & 100.5 \\
\hline Dallas, TX & 17059 & $(16621,17473)$ & 20063 & 1.18 & 3004 & $(2590,3442)$ & 113 & 2481 & 82.6 \\
\hline Denton, TX & 4266 & $(4107,4416)$ & 4863 & 1.14 & 597 & $(447,756)$ & 66 & 436 & 73.0 \\
\hline $\begin{array}{l}\text { Dimmit-La Salle-Maverick, } \\
\text { TX }\end{array}$ & 570 & $(514,628)$ & 874 & 1.53 & 304 & $(246,360)$ & 397 & 257 & 84.5 \\
\hline $\begin{array}{l}\text { Duval and Nearby Coun- } \\
\text { ties, TX }\end{array}$ & 783 & $(717,852)$ & 934 & 1.19 & 151 & $(82,217)$ & 193 & 166 & 109.9 \\
\hline $\begin{array}{l}\text { Eastern Central Panhan- } \\
\text { dle, TX }\end{array}$ & 780 & $(711,850)$ & 955 & 1.22 & 175 & $(105,244)$ & 312 & 146 & 83.4 \\
\hline Ector, TX & 1264 & $(1176,1350)$ & 1629 & 1.29 & 365 & $(279,453)$ & 212 & 289 & 79.2 \\
\hline Ellis, TX & 1439 & $(1348,1536)$ & 1675 & 1.16 & 236 & $(139,327)$ & 123 & 189 & 80.1 \\
\hline El Paso, TX & 5804 & $(5607,6000)$ & 8435 & 1.45 & 2631 & $(2435,2828)$ & 313 & 2173 & 82.6 \\
\hline Falls-Lee-Milam, TX & 707 & $(646,777)$ & 734 & 1.04 & 27 & $(-43,88)$ & 45 & 80 & 296.3 \\
\hline $\begin{array}{l}\text { Fannin-Lamar-Red River, } \\
\text { TX }\end{array}$ & 1318 & $(1225,1409)$ & 1537 & 1.17 & 219 & $(128,312)$ & 221 & 202 & 92.2 \\
\hline Fort Bend, TX & 3817 & $(3679,3962)$ & 4565 & 1.20 & 748 & $(603,886)$ & 90 & 461 & 61.6 \\
\hline $\begin{array}{l}\text { Freestone-Leon-Limestone- } \\
\text { Navarro, TX }\end{array}$ & 1354 & $(1261,1442)$ & 1506 & 1.11 & 152 & $(64,245)$ & 136 & 171 & 112.5 \\
\hline $\begin{array}{l}\text { Frio-Medina-Uvalde- } \\
\text { Zavala, TX }\end{array}$ & 1023 & $(947,1102)$ & 1197 & 1.17 & 174 & $(95,250)$ & 157 & 155 & 89.1 \\
\hline $\begin{array}{l}\text { Gaines and Nearby Coun- } \\
\text { ties, TX }\end{array}$ & 713 & $(653,779)$ & 947 & 1.33 & 234 & $(168,294)$ & 273 & 230 & 98.3 \\
\hline Galveston, TX & 3031 & $(2897,3177)$ & 3348 & 1.10 & 317 & $(171,451)$ & 92 & 239 & 75.4 \\
\hline Grayson, TX & 1581 & $(1486,1683)$ & 1795 & 1.14 & 214 & $(112,309)$ & 153 & 208 & 97.2 \\
\hline Gregg, TX & 1393 & $(1298,1495)$ & 1642 & 1.18 & 249 & $(147,344)$ & 200 & 187 & 75.1 \\
\hline Guadalupe, TX & 1257 & $(1169,1343)$ & 1380 & 1.10 & 123 & $(37,211)$ & 72 & 131 & 106.5 \\
\hline Hardin, TX & 626 & $(568,685)$ & 670 & 1.07 & 44 & $(-15,102)$ & 76 & 59 & 134.1 \\
\hline Harris, TX & 27882 & $(27348,28444)$ & 32980 & 1.18 & 5098 & $(4536,5632)$ & 107 & 3871 & 75.9 \\
\hline Harrison-Upshur, TX & 1204 & $(1122,1291)$ & 1346 & 1.12 & 142 & $(55,224)$ & 131 & 144 & 101.4 \\
\hline Hays, TX & 1297 & $(1212,1375)$ & 1349 & 1.04 & 52 & $(-26,137)$ & 22 & 110 & 211.5 \\
\hline Henderson, TX & 1221 & $(1136,1305)$ & 1326 & 1.09 & 105 & $(21,190)$ & 124 & 110 & 104.8 \\
\hline Hidalgo, TX & 4841 & $(4680,5022)$ & 7270 & 1.50 & 2429 & $(2248,2590)$ & 276 & 2097 & 86.3 \\
\hline Hood, TX & 844 & $(776,914)$ & 903 & 1.07 & 59 & $(-11,127)$ & 90 & 86 & 145.8 \\
\hline $\begin{array}{l}\text { Hopkins and Nearby Coun- } \\
\text { ties, TX }\end{array}$ & 1790 & $(1680,1891)$ & 2075 & 1.16 & 285 & $(184,395)$ & 194 & 277 & 97.2 \\
\hline Hunt, TX & 1063 & $(989,1146)$ & 1163 & 1.09 & 100 & $(17,174)$ & 98 & 101 & 101.0 \\
\hline $\begin{array}{l}\text { Jack-Palo Pinto-Young, } \\
\text { TX }\end{array}$ & 737 & $(669,804)$ & 894 & 1.21 & 157 & $(90,225)$ & 278 & 102 & 65.0 \\
\hline $\begin{array}{l}\text { Jackson-Matagorda- } \\
\text { Wharton, TX }\end{array}$ & 1038 & $(964,1118)$ & 1189 & 1.15 & 151 & $(71,225)$ & 164 & 179 & 118.5 \\
\hline Jasper-Newton, TX & 634 & $(567,691)$ & 745 & 1.18 & 111 & $(54,178)$ & 223 & 76 & 68.5 \\
\hline Jefferson, TX & 2561 & $(2422,2685)$ & 2967 & 1.16 & 406 & $(282,545)$ & 161 & 289 & 71.2 \\
\hline Johnson, TX & 1612 & $(1513,1707)$ & 1839 & 1.14 & 227 & $(132,326)$ & 126 & 183 & 80.6 \\
\hline Kaufman, TX & 1165 & $(1082,1250)$ & 1282 & 1.10 & 117 & $(32,200)$ & 83 & 143 & 122.2 \\
\hline $\begin{array}{l}\text { Kenedy-Kleberg-Willacy, } \\
\text { TX }\end{array}$ & 512 & $(458,567)$ & 608 & 1.19 & 96 & $(41,150)$ & 181 & 140 & 145.8 \\
\hline $\begin{array}{l}\text { Lavaca and Nearby Coun- } \\
\text { ties, TX }\end{array}$ & 1362 & $(1274,1454)$ & 1568 & 1.15 & 206 & $(114,294)$ & 191 & 194 & 94.2 \\
\hline Lubbock, TX & 2720 & $(2593,2855)$ & 3481 & 1.28 & 761 & $(626,888)$ & 244 & 664 & 87.3 \\
\hline
\end{tabular}


Table C1: Primary Estimates for Each County-Set

\begin{tabular}{|c|c|c|c|c|c|c|c|c|c|}
\hline County-Set & $\begin{array}{l}\text { Expected } \\
\text { Deaths }\end{array}$ & $\begin{array}{l}\text { Expected } \\
\text { Deaths } 95 \% \text { CI }\end{array}$ & $\begin{array}{l}\text { Observed } \\
\text { Deaths }\end{array}$ & $\begin{array}{l}\text { Observed/ } \\
\text { Expected }\end{array}$ & $\begin{array}{l}\text { Excess } \\
\text { Deaths }\end{array}$ & $\begin{array}{l}\text { Excess Deaths } \\
95 \% \text { CI }\end{array}$ & $\begin{array}{l}\text { Excess Deaths } \\
\text { Per } 100,000\end{array}$ & $\begin{array}{l}\text { COVID-19 } \\
\text { Deaths }\end{array}$ & $\begin{array}{l}\text { COVID-19 to } \\
\text { Excess Ratio (\%) }\end{array}$ \\
\hline McLennan, TX & 2335 & $(2219,2451)$ & 2748 & 1.18 & 413 & $(297,529)$ & 158 & 340 & 82.3 \\
\hline $\begin{array}{l}\text { Madison-Trinity-Walker, } \\
\text { TX }\end{array}$ & 977 & $(899,1053)$ & 1096 & 1.12 & 119 & $(43,197)$ & 117 & 136 & 114.3 \\
\hline $\begin{array}{l}\text { Menard and Nearby Coun- } \\
\text { ties, TX }\end{array}$ & 1306 & $(1216,1402)$ & 1420 & 1.09 & 114 & $(18,204)$ & 111 & 160 & 140.4 \\
\hline Midland, TX & 1211 & $(1127,1294)$ & 1316 & 1.09 & 105 & $(22,189)$ & 56 & 211 & 201.0 \\
\hline Montgomery, TX & 4337 & $(4168,4510)$ & 4688 & 1.08 & 351 & $(178,520)$ & 56 & 312 & 88.9 \\
\hline Nacogdoches, TX & 602 & $(546,660)$ & 760 & 1.26 & 158 & $(100,214)$ & 240 & 110 & 69.6 \\
\hline Northeast Panhandle, TX & 562 & $(504,622)$ & 657 & 1.17 & 95 & $(35,153)$ & 182 & 118 & 124.2 \\
\hline Northwest Panhandle, TX & 597 & $(539,658)$ & 795 & 1.33 & 198 & $(137,256)$ & 302 & 184 & 92.9 \\
\hline Nueces, TX & 3050 & $(2905,3194)$ & 3792 & 1.24 & 742 & $(598,887)$ & 203 & 527 & 71.0 \\
\hline Orange, TX & 1000 & $(928,1076)$ & 1152 & 1.15 & 152 & $(76,224)$ & 187 & 83 & 54.6 \\
\hline $\begin{array}{l}\text { Panola-Sabine-San } \\
\text { Augustine-Shelby, TX }\end{array}$ & 894 & $(822,968)$ & 1095 & 1.22 & 201 & $(127,273)$ & 297 & 144 & 71.6 \\
\hline Parker, TX & 1328 & $(1244,1419)$ & 1424 & 1.07 & 96 & $(5,180)$ & 65 & 127 & 132.3 \\
\hline $\begin{array}{l}\text { Polk-San Jacinto-Tyler, } \\
\text { TX }\end{array}$ & 1369 & $(1277,1465)$ & 1538 & 1.12 & 169 & $(73,261)$ & 162 & 115 & 68.0 \\
\hline Potter, TX & 1278 & $(1194,1372)$ & 1644 & 1.29 & 366 & $(272,450)$ & 310 & 310 & 84.7 \\
\hline Rains-Van Zandt, TX & 861 & $(790,929)$ & 1032 & 1.20 & 171 & $(103,242)$ & 243 & 99 & 57.9 \\
\hline Randall, TX & 1140 & $(1058,1219)$ & 1407 & 1.23 & 267 & $(188,349)$ & 191 & 217 & 81.3 \\
\hline Rockwall, TX & 692 & $(633,754)$ & 753 & 1.09 & 61 & $(-1,120)$ & 56 & 67 & 109.8 \\
\hline Rusk, TX & 551 & $(495,611)$ & 684 & 1.24 & 133 & $(73,189)$ & 244 & 83 & 62.4 \\
\hline San Patricio, TX & 646 & $(587,711)$ & 770 & 1.19 & 124 & $(59,183)$ & 187 & 107 & 86.3 \\
\hline $\begin{array}{l}\text { Scurry and Nearby Coun- } \\
\text { ties, TX }\end{array}$ & 918 & $(847,991)$ & 1170 & 1.27 & 252 & $(179,323)$ & 287 & 214 & 84.9 \\
\hline Smith, TX & 2246 & $(2125,2355)$ & 2735 & 1.22 & 489 & $(380,610)$ & 207 & 312 & 63.8 \\
\hline Starr, TX & 452 & $(402,502)$ & 721 & 1.60 & 269 & $(219,319)$ & 413 & 219 & 81.4 \\
\hline Tarrant, TX & 14099 & $(13763,14428)$ & 16416 & 1.16 & 2317 & $(1988,2653)$ & 108 & 1878 & 81.1 \\
\hline Taylor, TX & 1505 & $(1405,1600)$ & 1636 & 1.09 & 131 & $(36,231)$ & 94 & 243 & 185.5 \\
\hline Tom Green, TX & 1122 & $(1041,1209)$ & 1439 & 1.28 & 317 & $(230,398)$ & 266 & 202 & 63.7 \\
\hline Travis, TX & 6097 & $(5913,6282)$ & 6988 & 1.15 & 891 & $(706,1075)$ & 69 & 617 & 69.2 \\
\hline Victoria, TX & 850 & $(778,920)$ & 1040 & 1.22 & 190 & $(120,262)$ & 206 & 138 & 72.6 \\
\hline Webb, TX & 1485 & $(1399,1586)$ & 2185 & 1.47 & 700 & $(599,786)$ & 250 & 535 & 76.4 \\
\hline $\begin{array}{l}\text { Western Central Panhan- } \\
\text { dle, TX }\end{array}$ & 768 & $(703,837)$ & 1075 & 1.40 & 307 & $(238,372)$ & 375 & 316 & 102.9 \\
\hline $\begin{array}{l}\text { West Texas, excluding El } \\
\text { Paso, TX }\end{array}$ & 727 & $(664,788)$ & 932 & 1.28 & 205 & $(144,268)$ & 237 & 149 & 72.7 \\
\hline Williamson, TX & 3418 & $(3276,3545)$ & 3691 & 1.08 & 273 & $(146,415)$ & 45 & 280 & 102.6 \\
\hline Wise, TX & 741 & $(677,804)$ & 785 & 1.06 & 44 & $(-19,108)$ & 60 & 83 & 188.6 \\
\hline Beaver-Iron-Millard, UT & 540 & $(486,592)$ & 592 & 1.10 & 52 & $(0,106)$ & 67 & 30 & 57.7 \\
\hline Box Elder-Cache-Rich, UT & 1083 & $(1005,1160)$ & 1165 & 1.08 & 82 & $(5,160)$ & 43 & 65 & 79.3 \\
\hline $\begin{array}{l}\text { Carbon-Daggett- } \\
\text { Duchesne-Uintah, UT }\end{array}$ & 656 & $(596,712)$ & 732 & 1.12 & 76 & $(20,136)$ & 98 & 32 & 42.1 \\
\hline Davis, UT & 1983 & $(1878,2086)$ & 2213 & 1.12 & 230 & $(127,335)$ & 64 & 113 & 49.1 \\
\hline Juab-Sanpete-Tooele, UT & 722 & $(662,785)$ & 750 & 1.04 & 28 & $(-35,88)$ & 23 & 39 & 139.3 \\
\hline $\begin{array}{l}\text { Morgan-Summit-Wasatch, } \\
\text { UT }\end{array}$ & 391 & $(345,436)$ & 448 & 1.15 & 57 & $(12,103)$ & 62 & 28 & 49.1 \\
\hline Salt Lake, UT & 7187 & $(6972,7421)$ & 7887 & 1.10 & 700 & $(466,915)$ & 59 & 665 & 95.0 \\
\hline Utah (county), UT & 2851 & $(2717,2984)$ & 3098 & 1.09 & 247 & $(114,381)$ & 38 & 266 & 107.7 \\
\hline Washington, UT & 1579 & $(1483,1677)$ & 1745 & 1.11 & 166 & $(68,262)$ & 91 & 123 & 74.1 \\
\hline $\begin{array}{l}\text { Wayne and Nearby Coun- } \\
\text { ties, UT }\end{array}$ & 749 & $(687,819)$ & 766 & 1.02 & 17 & $(-53,79)$ & 22 & 75 & 441.2 \\
\hline Weber, UT & 1927 & $(1818,2030)$ & 2095 & 1.09 & 168 & $(65,277)$ & 63 & 124 & 73.8 \\
\hline Addison-Chittenden, VT & 1587 & $(1495,1673)$ & 1595 & 1.01 & 8 & $(-78,100)$ & 4 & 74 & 925.0 \\
\hline Bennington-Windham, VT & 963 & $(890,1043)$ & 945 & 0.98 & -18 & $(-98,55)$ & -23 & 16 & NA \\
\hline
\end{tabular}


Table C1: Primary Estimates for Each County-Set

\begin{tabular}{|c|c|c|c|c|c|c|c|c|c|}
\hline County-Set & $\begin{array}{l}\text { Expected } \\
\text { Deaths }\end{array}$ & $\begin{array}{l}\text { Expected } \\
\text { Deaths } 95 \% \mathrm{CI}\end{array}$ & $\begin{array}{l}\text { Observed } \\
\text { Deaths }\end{array}$ & $\begin{array}{l}\text { Observed/ } \\
\text { Expected }\end{array}$ & $\begin{array}{l}\text { Excess } \\
\text { Deaths }\end{array}$ & $\begin{array}{l}\text { Excess Deaths } \\
95 \% \mathrm{CI}\end{array}$ & $\begin{array}{l}\text { Excess Deaths } \\
\text { Per } 100,000\end{array}$ & $\begin{array}{l}\text { COVID-19 } \\
\text { Deaths }\end{array}$ & $\begin{array}{c}\text { COVID-19 to } \\
\text { Excess Ratio (\%) }\end{array}$ \\
\hline $\begin{array}{l}\text { Caledonia-Essex-Orange, } \\
\text { VT }\end{array}$ & 721 & $(655,785)$ & 697 & 0.97 & -24 & $(-88,42)$ & -36 & 15 & NA \\
\hline Franklin-Grand Isle, VT & 535 & $(482,588)$ & 539 & 1.01 & 4 & $(-49,57)$ & 8 & 34 & 850.0 \\
\hline Lamoille-Orleans, VT & 539 & $(478,595)$ & 588 & 1.09 & 49 & $(-7,110)$ & 94 & 10 & 20.4 \\
\hline Rutland, VT & 743 & $(676,810)$ & 781 & 1.05 & 38 & $(-29,105)$ & 66 & 5 & 13.2 \\
\hline Washington, VT & 564 & $(508,621)$ & 644 & 1.14 & 80 & $(23,136)$ & 138 & 13 & 16.2 \\
\hline Windsor, VT & 618 & $(562,674)$ & 670 & 1.08 & 52 & $(-4,108)$ & 93 & 5 & 9.6 \\
\hline $\begin{array}{l}\text { Accomack-Northampton- } \\
\text { Virginia Beach (city), } \\
\text { VA }\end{array}$ & 3936 & $(3775,4100)$ & 4367 & 1.11 & 431 & $(267,592)$ & 87 & 245 & 56.8 \\
\hline $\begin{array}{l}\text { Albemarle-Charlottesville } \\
\text { (city), VA }\end{array}$ & 1215 & $(1133,1304)$ & 1326 & 1.09 & 111 & $(22,193)$ & 70 & 75 & 67.6 \\
\hline Alexandria (city), VA & 728 & $(663,798)$ & 833 & 1.14 & 105 & $(35,170)$ & 65 & 88 & 83.8 \\
\hline $\begin{array}{l}\text { Alleghany and Nearby } \\
\text { Counties and Independent } \\
\text { Cities, VA }\end{array}$ & 836 & $(769,901)$ & 863 & 1.03 & 27 & $(-38,94)$ & 45 & 57 & 211.1 \\
\hline $\begin{array}{l}\text { Amelia-Brunswick- } \\
\text { Lunenburg-Nottoway, } \\
\text { VA }\end{array}$ & 779 & $(709,851)$ & 808 & 1.04 & 29 & $(-43,99)$ & 51 & 55 & 189.7 \\
\hline $\begin{array}{l}\text { Amherst-Lynchburg (city)- } \\
\text { Nelson, VA }\end{array}$ & 1392 & $(1303,1488)$ & 1531 & 1.10 & 139 & $(43,228)$ & 106 & 84 & 60.4 \\
\hline $\begin{array}{l}\text { Appomattox-Campbell, } \\
\text { VA }\end{array}$ & 826 & $(756,895)$ & 869 & 1.05 & 43 & $(-26,113)$ & 61 & 53 & 123.3 \\
\hline Arlington, VA & 881 & $(809,950)$ & 1162 & 1.32 & 281 & $(212,353)$ & 116 & 158 & 56.2 \\
\hline $\begin{array}{l}\text { Augusta-Rockbridge and } \\
\text { Independent Cities, VA }\end{array}$ & 1916 & $(1819,2027)$ & 2033 & 1.06 & 117 & $(6,214)$ & 73 & 145 & 123.9 \\
\hline $\begin{array}{l}\text { Bedford (county)-Bedford } \\
\text { (city), VA }\end{array}$ & 932 & $(857,1001)$ & 961 & 1.03 & 29 & $(-40,104)$ & 37 & 45 & 155.2 \\
\hline Bland-Smyth-Wythe, VA & 998 & $(923,1077)$ & 1101 & 1.10 & 103 & $(24,178)$ & 160 & 106 & 102.9 \\
\hline $\begin{array}{l}\text { Bristol (city)-Washington, } \\
\text { VA }\end{array}$ & 968 & $(890,1047)$ & 1118 & 1.15 & 150 & $(71,228)$ & 213 & 90 & 60.0 \\
\hline $\begin{array}{l}\text { Buchanan-Dickenson- } \\
\text { Russell-Tazewell, VA }\end{array}$ & 1514 & $(1420,1626)$ & 1726 & 1.14 & 212 & $(100,306)$ & 210 & 106 & 50.0 \\
\hline $\begin{array}{l}\text { Carroll-Galax } \\
\text { Grayson, VA }\end{array}$ & 758 & $(690,824)$ & 874 & 1.15 & 116 & $(50,184)$ & 226 & 113 & 97.4 \\
\hline $\begin{array}{l}\text { Charles City-Goochland- } \\
\text { Henrico-New Kent, VA }\end{array}$ & 3504 & $(3356,3659)$ & 4054 & 1.16 & 550 & $(395,698)$ & 142 & 327 & 59.5 \\
\hline Chesapeake (city), VA & 1966 & $(1846,2070)$ & 2186 & 1.11 & 220 & $(116,340)$ & 89 & 146 & 66.4 \\
\hline $\begin{array}{l}\text { Chesterfield-Colonial } \\
\text { Heights (city)-Powhatan, } \\
\text { VA }\end{array}$ & 3410 & $(3259,3568)$ & 3638 & 1.07 & 228 & $(70,379)$ & 56 & 221 & 96.9 \\
\hline Clarke-Warren, VA & 588 & $(529,647)$ & 633 & 1.08 & 45 & $(-14,104)$ & 80 & 42 & 93.3 \\
\hline $\begin{array}{l}\text { Craig-Franklin (county)- } \\
\text { Roanoke (county)-Salem } \\
\text { (city), VA }\end{array}$ & 2179 & $(2062,2296)$ & 2395 & 1.10 & 216 & $(99,333)$ & 119 & 174 & 80.6 \\
\hline $\begin{array}{l}\text { Culpeper-Madison- } \\
\text { Rappahannock, VA }\end{array}$ & 718 & $(652,782)$ & 690 & 0.96 & -28 & $(-92,38)$ & -38 & 47 & NA \\
\hline $\begin{array}{l}\text { Danville } \\
\text { Pittsylvania, VA }\end{array}$ & 1442 & $(1342,1540)$ & 1615 & 1.12 & 173 & $(75,273)$ & 173 & 137 & 79.2 \\
\hline $\begin{array}{l}\text { Dinwiddie-Petersburg } \\
\text { (city), VA }\end{array}$ & 933 & $(855,1012)$ & 949 & 1.02 & 16 & $(-63,94)$ & 26 & 59 & 368.8 \\
\hline $\begin{array}{l}\text { Fairfax (county)-Fairfax } \\
\text { (city)-Falls Church (city), } \\
\text { VA }\end{array}$ & 5736 & $(5545,5923)$ & 6684 & 1.17 & 948 & $(761,1139)$ & 79 & 764 & 80.6 \\
\hline Fauquier, VA & 599 & $(543,661)$ & 620 & 1.04 & 21 & $(-41,77)$ & 29 & 27 & 128.6 \\
\hline
\end{tabular}


Table C1: Primary Estimates for Each County-Set

\begin{tabular}{|c|c|c|c|c|c|c|c|c|c|}
\hline County-Set & $\begin{array}{l}\text { Expected } \\
\text { Deaths }\end{array}$ & $\begin{array}{l}\text { Expected } \\
\text { Deaths } 95 \% \text { CI }\end{array}$ & $\begin{array}{l}\text { Observed } \\
\text { Deaths }\end{array}$ & $\begin{array}{l}\text { Observed/ } \\
\text { Expected }\end{array}$ & $\begin{array}{l}\text { Excess } \\
\text { Deaths }\end{array}$ & $\begin{array}{l}\text { Excess Deaths } \\
95 \% \text { CI }\end{array}$ & $\begin{array}{l}\text { Excess Deaths } \\
\text { Per } 100,000\end{array}$ & $\begin{array}{l}\text { COVID-19 } \\
\text { Deaths }\end{array}$ & $\begin{array}{l}\text { COVID-19 to } \\
\text { Excess Ratio (\%) }\end{array}$ \\
\hline $\begin{array}{l}\text { Floyd-Giles-Montgomery, } \\
\text { VA }\end{array}$ & 1083 & $(1007,1163)$ & 1256 & 1.16 & 173 & $(93,249)$ & 130 & 84 & 48.6 \\
\hline $\begin{array}{l}\text { Frederick-Winchester } \\
\text { (city), VA }\end{array}$ & 1091 & $(1014,1173)$ & 1217 & 1.12 & 126 & $(44,203)$ & 105 & 81 & 64.3 \\
\hline $\begin{array}{l}\text { Fredericksburg (city)- } \\
\text { Spotsylvania, VA }\end{array}$ & 1266 & $(1183,1349)$ & 1387 & 1.10 & 121 & $(38,204)$ & 72 & 87 & 71.9 \\
\hline Greene-Louisa-Orange, VA & 1018 & $(937,1102)$ & 1041 & 1.02 & 23 & $(-61,104)$ & 24 & 33 & 143.5 \\
\hline Halifax-Mecklenburg, VA & 992 & $(915,1072)$ & 1104 & 1.11 & 112 & $(32,189)$ & 176 & 122 & 108.9 \\
\hline $\begin{array}{l}\text { Hampton (city)-Poquoson } \\
\text { (city), VA }\end{array}$ & 1534 & $(1440,1632)$ & 1595 & 1.04 & 61 & $(-37,155)$ & 42 & 82 & 134.4 \\
\hline Hanover, VA & 1041 & $(970,1118)$ & 1136 & 1.09 & 95 & $(18,166)$ & 86 & 83 & 87.4 \\
\hline $\begin{array}{l}\text { Harrisonburg (city)- } \\
\text { Rockingham, VA }\end{array}$ & 1117 & $(1041,1194)$ & 1263 & 1.13 & 146 & $(69,222)$ & 106 & 129 & 88.4 \\
\hline $\begin{array}{l}\text { Henry-Martinsville (city)- } \\
\text { Patrick, VA }\end{array}$ & 1319 & $(1227,1411)$ & 1487 & 1.13 & 168 & $(76,260)$ & 208 & 157 & 93.5 \\
\hline $\begin{array}{l}\text { Hopewell (city)-Prince } \\
\text { George, VA }\end{array}$ & 589 & $(538,648)$ & 621 & 1.05 & 32 & $(-27,83)$ & 52 & 32 & 100.0 \\
\hline $\begin{array}{l}\text { James City -Williamsburg } \\
\text { (city), VA }\end{array}$ & 935 & $(866,1005)$ & 1013 & 1.08 & 78 & $(8,147)$ & 84 & 43 & 55.1 \\
\hline $\begin{array}{l}\text { King and Queen and } \\
\text { Nearby Counties, VA }\end{array}$ & 1487 & $(1399,1594)$ & 1628 & 1.09 & 141 & $(34,229)$ & 114 & 74 & 52.5 \\
\hline $\begin{array}{l}\text { Lee-Norton (city)-Scott- } \\
\text { Wise, VA }\end{array}$ & 1225 & $(1143,1308)$ & 1420 & 1.16 & 195 & $(112,277)$ & 228 & 135 & 69.2 \\
\hline Loudoun, VA & 1635 & $(1539,1723)$ & 1864 & 1.14 & 229 & $(141,325)$ & 54 & 184 & 80.3 \\
\hline $\begin{array}{l}\text { Manassas (city)-Manassas } \\
\text { Park (city), VA }\end{array}$ & 325 & $(284,367)$ & 327 & 1.01 & 2 & $(-40,43)$ & 4 & 53 & 2650.0 \\
\hline Newport News (city), VA & 1641 & $(1549,1738)$ & 1899 & 1.16 & 258 & $(161,350)$ & 146 & 98 & 38.0 \\
\hline Norfolk (city), VA & 2080 & $(1971,2189)$ & 2237 & 1.08 & 157 & $(48,266)$ & 65 & 134 & 85.4 \\
\hline Page-Shenandoah, VA & 830 & $(757,907)$ & 1005 & 1.21 & 175 & $(98,248)$ & 257 & 106 & 60.6 \\
\hline Portsmouth (city), VA & 1085 & $(1007,1172)$ & 1264 & 1.16 & 179 & $(92,257)$ & 190 & 99 & 55.3 \\
\hline $\begin{array}{l}\text { Prince Edward and Nearby } \\
\text { Counties, VA }\end{array}$ & 948 & $(876,1031)$ & 1047 & 1.10 & 99 & $(16,171)$ & 111 & 76 & 76.8 \\
\hline Prince William, VA & 2203 & $(2096,2305)$ & 2702 & 1.23 & 499 & $(397,606)$ & 105 & 345 & 69.1 \\
\hline Pulaski-Radford (city), VA & 612 & $(555,673)$ & 672 & 1.10 & 60 & $(-1,117)$ & 115 & 58 & 96.7 \\
\hline Richmond (city), VA & 1836 & $(1733,1942)$ & 2040 & 1.11 & 204 & $(98,307)$ & 88 & 148 & 72.5 \\
\hline Roanoke (city), VA & 1279 & $(1194,1371)$ & 1501 & 1.17 & 222 & $(130,307)$ & 220 & 123 & 55.4 \\
\hline $\begin{array}{l}\text { Southampton and Nearby } \\
\text { Counties and Independent } \\
\text { Cities, VA }\end{array}$ & 1137 & $(1054,1222)$ & 1327 & 1.17 & 190 & $(105,273)$ & 197 & 157 & 82.6 \\
\hline Stafford, VA & 900 & $(828,971)$ & 901 & 1.00 & 1 & $(-70,73)$ & 1 & 38 & 3800.0 \\
\hline Suffolk (city), VA & 803 & $(735,871)$ & 977 & 1.22 & 174 & $(106,242)$ & 186 & 92 & 52.9 \\
\hline $\begin{array}{l}\text { Westmoreland and Nearby } \\
\text { Counties, VA }\end{array}$ & 997 & $(924,1080)$ & 1082 & 1.09 & 85 & $(2,158)$ & 110 & 58 & 68.2 \\
\hline York, VA & 486 & $(439,542)$ & 534 & 1.10 & 48 & $(-8,95)$ & 71 & 24 & 50.0 \\
\hline Adams-Grant, WA & 857 & $(790,929)$ & 952 & 1.11 & 95 & $(23,162)$ & 79 & 110 & 115.8 \\
\hline $\begin{array}{l}\text { Asotin-Columbia-Garfield- } \\
\text { Whitman, WA }\end{array}$ & 637 & $(577,697)$ & 717 & 1.13 & 80 & $(20,140)$ & 101 & 62 & 77.5 \\
\hline Benton, WA & 1581 & $(1488,1677)$ & 1784 & 1.13 & 203 & $(107,296)$ & 97 & 168 & 82.8 \\
\hline Chelan-Kittitas, WA & 1075 & $(988,1158)$ & 1122 & 1.04 & 47 & $(-36,134)$ & 37 & 76 & 161.7 \\
\hline Clallam-Jefferson, WA & 1520 & $(1423,1624)$ & 1498 & 0.99 & -22 & $(-126,75)$ & -20 & 10 & NA \\
\hline Clark, WA & 3873 & $(3710,4029)$ & 4194 & 1.08 & 321 & $(165,484)$ & 65 & 144 & 44.9 \\
\hline $\begin{array}{l}\text { Cowlitz-Klickitat- } \\
\text { Skamania-Wahkiakum, } \\
\text { WA }\end{array}$ & 1674 & $(1563,1778)$ & 1692 & 1.01 & 18 & $(-86,129)$ & 12 & 43 & 238.9 \\
\hline Douglas-Okanogan, WA & 788 & $(719,860)$ & 828 & 1.05 & 40 & $(-32,109)$ & 46 & 51 & 127.5 \\
\hline
\end{tabular}


Table C1: Primary Estimates for Each County-Set

\begin{tabular}{|c|c|c|c|c|c|c|c|c|c|}
\hline County-Set & $\begin{array}{l}\text { Expected } \\
\text { Deaths }\end{array}$ & $\begin{array}{l}\text { Expected } \\
\text { Deaths } 95 \% \text { CI }\end{array}$ & $\begin{array}{l}\text { Observed } \\
\text { Deaths }\end{array}$ & $\begin{array}{l}\text { Observed/ } \\
\text { Expected }\end{array}$ & $\begin{array}{l}\text { Excess } \\
\text { Deaths }\end{array}$ & $\begin{array}{l}\text { Excess Deaths } \\
95 \% \mathrm{CI}\end{array}$ & $\begin{array}{l}\text { Excess Deaths } \\
\text { Per } 100,000\end{array}$ & $\begin{array}{l}\text { COVID-19 } \\
\text { Deaths }\end{array}$ & $\begin{array}{l}\text { COVID-19 to } \\
\text { Excess Ratio (\%) }\end{array}$ \\
\hline $\begin{array}{l}\text { Ferry-Lincoln-Pend } \\
\text { Oreille-Stevens, WA }\end{array}$ & 887 & $(821,963)$ & 922 & 1.04 & 35 & $(-41,101)$ & 43 & 38 & 108.6 \\
\hline Franklin, WA & 439 & $(386,488)$ & 528 & 1.20 & 89 & $(40,142)$ & 90 & 78 & 87.6 \\
\hline Grays Harbor-Pacific, WA & 1303 & $(1214,1402)$ & 1288 & 0.99 & -15 & $(-114,74)$ & -16 & 35 & NA \\
\hline Island, WA & 869 & $(801,939)$ & 855 & 0.98 & -14 & $(-84,54)$ & -16 & 34 & NA \\
\hline King, WA & 13653 & $(13293,14032)$ & 14510 & 1.06 & 857 & $(478,1217)$ & 37 & 1093 & 127.5 \\
\hline Kitsap, WA & 2438 & $(2310,2560)$ & 2364 & 0.97 & -74 & $(-196,54)$ & -27 & 50 & NA \\
\hline Lewis, WA & 993 & $(919,1076)$ & 1023 & 1.03 & 30 & $(-53,104)$ & 36 & 32 & 106.7 \\
\hline Mason, WA & 757 & $(691,823)$ & 746 & 0.99 & -11 & $(-77,55)$ & -16 & 17 & NA \\
\hline Pierce, WA & 7369 & $(7156,7609)$ & 7490 & 1.02 & 121 & $(-119,334)$ & 13 & 353 & 291.7 \\
\hline San Juan-Skagit, WA & 1480 & $(1383,1569)$ & 1510 & 1.02 & 30 & $(-59,127)$ & 20 & 52 & 173.3 \\
\hline Snohomish, WA & 5904 & $(5710,6102)$ & 6206 & 1.05 & 302 & $(104,496)$ & 36 & 408 & 135.1 \\
\hline Spokane, WA & 5012 & $(4821,5194)$ & 5297 & 1.06 & 285 & $(103,476)$ & 53 & 352 & 123.5 \\
\hline Thurston, WA & 2537 & $(2422,2671)$ & 2533 & 1.00 & -4 & $(-138,111)$ & -1 & 63 & NA \\
\hline Walla Walla, WA & 619 & $(560,679)$ & 642 & 1.04 & 23 & $(-37,82)$ & 37 & 43 & 187.0 \\
\hline Whatcom, WA & 1827 & $(1724,1935)$ & 1903 & 1.04 & 76 & $(-32,179)$ & 32 & 65 & 85.5 \\
\hline Yakima, WA & 2163 & $(2047,2279)$ & 2535 & 1.17 & 372 & $(256,488)$ & 147 & 335 & 90.1 \\
\hline $\begin{array}{l}\text { Barbour-Pocahontas- } \\
\text { Randolph-Tucker, WV }\end{array}$ & 765 & $(701,832)$ & 886 & 1.16 & 121 & $(54,185)$ & 201 & 28 & 23.1 \\
\hline Berkeley-Morgan, WV & 1424 & $(1334,1522)$ & 1613 & 1.13 & 189 & $(91,279)$ & 135 & 78 & 41.3 \\
\hline Boone-Lincoln-Logan, WV & 1195 & $(1101,1286)$ & 1268 & 1.06 & 73 & $(-18,167)$ & 100 & 95 & 130.1 \\
\hline $\begin{array}{l}\text { Braxton-Gilmer-Lewis- } \\
\text { Upshur, WV }\end{array}$ & 814 & $(744,883)$ & 839 & 1.03 & 25 & $(-44,95)$ & 40 & 20 & 80.0 \\
\hline Brooke-Hancock, WV & 770 & $(702,840)$ & 937 & 1.22 & 167 & $(97,235)$ & 333 & 108 & 64.7 \\
\hline Cabell, WV & 1368 & $(1270,1466)$ & 1371 & 1.00 & 3 & $(-95,101)$ & 3 & 104 & 3466.7 \\
\hline $\begin{array}{l}\text { Clay-Fayette-Nicholas- } \\
\text { Webster, WV }\end{array}$ & 1237 & $(1146,1331)$ & 1299 & 1.05 & 62 & $(-32,153)$ & 75 & 64 & 103.2 \\
\hline $\begin{array}{l}\text { Greenbrier-Monroe- } \\
\text { Summers, WV }\end{array}$ & 873 & $(796,948)$ & 989 & 1.13 & 116 & $(41,193)$ & 195 & 77 & 66.4 \\
\hline $\begin{array}{l}\text { Hardy and Nearby Coun- } \\
\text { ties, WV }\end{array}$ & 1042 & $(960,1121)$ & 1223 & 1.17 & 181 & $(102,263)$ & 221 & 130 & 71.8 \\
\hline Harrison-Taylor, WV & 1067 & $(986,1152)$ & 1114 & 1.04 & 47 & $(-38,128)$ & 56 & 43 & 91.5 \\
\hline $\begin{array}{l}\text { Jackson and Nearby Coun- } \\
\text { ties, WV }\end{array}$ & 1159 & $(1083,1236)$ & 1167 & 1.01 & 8 & $(-69,84)$ & 9 & 69 & 862.5 \\
\hline Jefferson, WV & 541 & $(487,600)$ & 613 & 1.13 & 72 & $(13,126)$ & 125 & 36 & 50.0 \\
\hline Kanawha, WV & 2627 & $(2490,2762)$ & 2943 & 1.12 & 316 & $(181,453)$ & 181 & 217 & 68.7 \\
\hline McDowell-Mercer, WV & 1196 & $(1112,1289)$ & 1402 & 1.17 & 206 & $(113,290)$ & 273 & 59 & 28.6 \\
\hline Marion, WV & 747 & $(681,811)$ & 735 & 0.98 & -12 & $(-76,54)$ & -22 & 20 & NA \\
\hline Marshall-Ohio, WV & 973 & $(899,1053)$ & 1153 & 1.18 & 180 & $(100,254)$ & 254 & 110 & 61.1 \\
\hline Mingo-Wayne, WV & 924 & $(850,996)$ & 1043 & 1.13 & 119 & $(47,193)$ & 191 & 61 & 51.3 \\
\hline Monongalia-Preston, WV & 1142 & $(1057,1226)$ & 1246 & 1.09 & 104 & $(20,189)$ & 74 & 71 & 68.3 \\
\hline Putnam, WV & 655 & $(595,720)$ & 690 & 1.05 & 35 & $(-30,95)$ & 61 & 58 & 165.7 \\
\hline Raleigh-Wyoming, WV & 1411 & $(1321,1517)$ & 1590 & 1.13 & 179 & $(73,269)$ & 193 & 58 & 32.4 \\
\hline $\begin{array}{l}\text { Tyler and Nearby Coun- } \\
\text { ties, WV }\end{array}$ & 629 & $(572,690)$ & 660 & 1.05 & 31 & $(-30,88)$ & 63 & 34 & 109.7 \\
\hline Wood, WV & 1141 & $(1054,1226)$ & 1201 & 1.05 & 60 & $(-25,147)$ & 73 & 81 & 135.0 \\
\hline $\begin{array}{l}\text { Adams-Juneau-Monroe- } \\
\text { Vernon, WI }\end{array}$ & 1362 & $(1269,1450)$ & 1471 & 1.08 & 109 & $(21,202)$ & 87 & 99 & 90.8 \\
\hline $\begin{array}{l}\text { Ashland-Bayfield-Douglas- } \\
\text { Price, WI }\end{array}$ & 999 & $(919,1075)$ & 1155 & 1.16 & 156 & $(80,236)$ & 179 & 106 & 67.9 \\
\hline $\begin{array}{l}\text { Barron-Sawyer-Washburn, } \\
\text { WI }\end{array}$ & 968 & $(893,1041)$ & 1099 & 1.14 & 131 & $(58,206)$ & 168 & 102 & 77.9 \\
\hline Brown, WI & 2184 & $(2064,2297)$ & 2453 & 1.12 & 269 & $(156,389)$ & 101 & 255 & 94.8 \\
\hline $\begin{array}{l}\text { Buffalo-Jackson-Pepin- } \\
\text { Trempealeau, WI }\end{array}$ & 734 & $(669,797)$ & 798 & 1.09 & 64 & $(1,129)$ & 90 & 73 & 114.1 \\
\hline Burnett-Polk, WI & 715 & $(648,781)$ & 701 & 0.98 & -14 & $(-80,53)$ & -23 & 61 & NA \\
\hline
\end{tabular}


Table C1: Primary Estimates for Each County-Set

\begin{tabular}{|c|c|c|c|c|c|c|c|c|c|}
\hline County-Set & $\begin{array}{l}\text { Expected } \\
\text { Deaths }\end{array}$ & $\begin{array}{l}\text { Expected } \\
\text { Deaths } 95 \% \text { CI }\end{array}$ & $\begin{array}{l}\text { Observed } \\
\text { Deaths }\end{array}$ & $\begin{array}{l}\text { Observed/ } \\
\text { Expected }\end{array}$ & $\begin{array}{l}\text { Excess } \\
\text { Deaths }\end{array}$ & $\begin{array}{l}\text { Excess Deaths } \\
95 \% \mathrm{CI}\end{array}$ & $\begin{array}{l}\text { Excess Deaths } \\
\text { Per } 100,000\end{array}$ & $\begin{array}{l}\text { COVID-19 } \\
\text { Deaths }\end{array}$ & $\begin{array}{l}\text { COVID-19 to } \\
\text { Excess Ratio (\%) }\end{array}$ \\
\hline Calumet-Winnebago, WI & 2004 & $(1895,2116)$ & 2227 & 1.11 & 223 & $(111,332)$ & 100 & 236 & 105.8 \\
\hline Chippewa-Rusk, WI & 846 & $(781,917)$ & 888 & 1.05 & 42 & $(-29,107)$ & 53 & 86 & 204.8 \\
\hline Clark-Taylor, WI & 566 & $(510,628)$ & 601 & 1.06 & 35 & $(-27,91)$ & 62 & 92 & 262.9 \\
\hline Columbia, WI & 580 & $(521,640)$ & 634 & 1.09 & 54 & $(-6,113)$ & 94 & 48 & 88.9 \\
\hline $\begin{array}{l}\text { Crawford-Grant-Richland, } \\
\text { WI }\end{array}$ & 893 & $(828,965)$ & 1022 & 1.14 & 129 & $(57,194)$ & 152 & 110 & 85.3 \\
\hline Dane, WI & 3526 & $(3379,3662)$ & 3984 & 1.13 & 458 & $(322,605)$ & 83 & 247 & 53.9 \\
\hline Dodge, WI & 1005 & $(931,1081)$ & 1210 & 1.20 & 205 & $(129,279)$ & 232 & 156 & 76.1 \\
\hline $\begin{array}{l}\text { Door-Kewaunee- } \\
\text { Manitowoc, WI }\end{array}$ & 1486 & $(1395,1577)$ & 1631 & 1.10 & 145 & $(54,236)$ & 114 & 127 & 87.6 \\
\hline Dunn-Eau Claire, WI & 1331 & $(1245,1420)$ & 1385 & 1.04 & 54 & $(-35,140)$ & 35 & 114 & 211.1 \\
\hline $\begin{array}{l}\text { Florence-Forest-Marinette- } \\
\text { Oconto, WI }\end{array}$ & 1175 & $(1096,1260)$ & 1309 & 1.11 & 134 & $(49,213)$ & 145 & 169 & 126.1 \\
\hline Fond du Lac, WI & 1015 & $(939,1093)$ & 1167 & 1.15 & 152 & $(74,228)$ & 146 & 115 & 75.7 \\
\hline Green-Iowa-Lafayette, WI & 720 & $(654,785)$ & 826 & 1.15 & 106 & $(41,172)$ & 136 & 33 & 31.1 \\
\hline $\begin{array}{l}\text { Green Lake-Marquette- } \\
\text { Waushara, WI }\end{array}$ & 776 & $(708,842)$ & 843 & 1.09 & 67 & $(1,135)$ & 114 & 71 & 106.0 \\
\hline Iron-Oneida-Vilas, WI & 909 & $(830,983)$ & 1013 & 1.11 & 104 & $(30,183)$ & 163 & 107 & 102.9 \\
\hline Jefferson, WI & 786 & $(724,847)$ & 863 & 1.10 & 77 & $(16,139)$ & 89 & 90 & 116.9 \\
\hline Kenosha, WI & 1532 & $(1433,1631)$ & 1746 & 1.14 & 214 & $(115,313)$ & 125 & 261 & 122.0 \\
\hline La Crosse, WI & 1064 & $(989,1138)$ & 1185 & 1.11 & 121 & $(47,196)$ & 102 & 76 & 62.8 \\
\hline $\begin{array}{l}\text { Langlade-Lincoln- } \\
\text { Menominee-Shawano, } \\
\text { WI }\end{array}$ & 1139 & $(1060,1225)$ & 1356 & 1.19 & 217 & $(131,296)$ & 235 & 206 & 94.9 \\
\hline Marathon, WI & 1284 & $(1196,1371)$ & 1433 & 1.12 & 149 & $(62,237)$ & 110 & 188 & 126.2 \\
\hline Milwaukee, WI & 8590 & $(8321,8854)$ & 10377 & 1.21 & 1787 & $(1523,2056)$ & 190 & 1076 & 60.2 \\
\hline Outagamie, WI & 1599 & $(1503,1690)$ & 1757 & 1.10 & 158 & $(67,254)$ & 83 & 186 & 117.7 \\
\hline Ozaukee, WI & 861 & $(792,934)$ & 937 & 1.09 & 76 & $(3,145)$ & 84 & 89 & 117.1 \\
\hline Pierce-St. Croix, WI & 988 & $(918,1065)$ & 1098 & 1.11 & 110 & $(33,180)$ & 81 & 76 & 69.1 \\
\hline Portage, WI & 559 & $(505,615)$ & 624 & 1.12 & 65 & $(9,119)$ & 90 & 51 & 78.5 \\
\hline Racine, WI & 1999 & $(1893,2110)$ & 2298 & 1.15 & 299 & $(188,405)$ & 151 & 288 & 96.3 \\
\hline Rock, WI & 1628 & $(1528,1731)$ & 1855 & 1.14 & 227 & $(124,327)$ & 138 & 154 & 67.8 \\
\hline Sauk, WI & 691 & $(632,759)$ & 747 & 1.08 & 56 & $(-12,115)$ & 86 & 46 & 82.1 \\
\hline Sheboygan, WI & 1169 & $(1086,1253)$ & 1337 & 1.14 & 168 & $(84,251)$ & 145 & 148 & 88.1 \\
\hline Walworth, WI & 1005 & $(929,1083)$ & 1196 & 1.19 & 191 & $(113,267)$ & 182 & 133 & 69.6 \\
\hline Washington, WI & 1277 & $(1197,1362)$ & 1393 & 1.09 & 116 & $(31,196)$ & 85 & 127 & 109.5 \\
\hline Waukesha, WI & 3772 & $(3611,3937)$ & 4277 & 1.13 & 505 & $(340,666)$ & 124 & 477 & 94.5 \\
\hline Waupaca, WI & 745 & $(678,819)$ & 824 & 1.11 & 79 & $(5,146)$ & 154 & 125 & 158.2 \\
\hline Wood, WI & 892 & $(821,966)$ & 984 & 1.10 & 92 & $(18,163)$ & 126 & 62 & 67.4 \\
\hline $\begin{array}{l}\text { Big Horn-Hot Springs- } \\
\text { Park-Washakie, WY }\end{array}$ & 624 & $(563,686)$ & 704 & 1.13 & 80 & $(18,141)$ & 151 & 68 & 85.0 \\
\hline $\begin{array}{l}\text { Fremont-Lincoln-Sublette- } \\
\text { Teton, WY }\end{array}$ & 748 & $(689,816)$ & 906 & 1.21 & 158 & $(90,217)$ & 173 & 90 & 57.0 \\
\hline Laramie, WY & 903 & $(832,974)$ & 982 & 1.09 & 79 & $(8,150)$ & 79 & 71 & 89.9 \\
\hline Natrona, WY & 742 & $(677,807)$ & 924 & 1.25 & 182 & $(117,247)$ & 233 & 110 & 60.4 \\
\hline Northeast Wyoming, WY & 846 & $(776,911)$ & 963 & 1.14 & 117 & $(52,187)$ & 118 & 82 & 70.1 \\
\hline $\begin{array}{l}\text { Southeast Wyoming, ex- } \\
\text { cluding Laramie, WY }\end{array}$ & 742 & $(678,811)$ & 909 & 1.23 & 167 & $(98,231)$ & 183 & 74 & 44.3 \\
\hline Sweetwater-Uinta, WY & 486 & $(435,538)$ & 595 & 1.22 & 109 & $(57,160)$ & 176 & 31 & 28.4 \\
\hline
\end{tabular}

